\title{
Equisingular deformations of plane curves in arbitrary characteristic
}

\author{
Antonio Campillo, Gert-Martin Greuel and Christoph Lossen \\ Dedicated to Jozef Steenbrink on the occasion of his 60th birthday
}

\begin{abstract}
The purpose of this paper is to develop the theory of equisingular deformations of plane curve singularities in arbitrary characteristic. We study equisingular deformations of the parametrization and of the equation and show that the base space of its semiuniversal deformation is smooth in both cases. Our approach through deformations of the parametrization is elementary and we show that equisingular deformations of the parametrization form a linear subfunctor of all deformations of the parametrization. This gives additional information, even in characteristic zero, the case which was treated by J. Wahl. The methods and proofs extend easily to good characteristic, that is, when the characteristic does not divide the multiplicity of any branch of the singularity. In bad characteristic, however, new phenomena occur and we are naturally led to consider weakly trivial (respectively, weakly equisingular) deformations, that is, deformations which become trivial (respectively, equisingular) after a finite and dominant base change. The semiuniversal base space for weakly equisingular deformations is, in general, not smooth but becomes smooth after a finite and purely inseparable base extension. The proof of this fact is more complicated and we introduce new constructions which may have further applications in the theory of singularities in positive characteristic.
\end{abstract}

\section{Contents}

Introduction . . . . . . . . . . . . . . . . . . . . . . 830

1 Deformations of the parametrization and of the normalization . . . . 832

2 Equisingular deformations of the parametrization . . . . . . . . . 835

3 Versal equisingular deformations of the parametrization . . . . . . . 841

4 Equisingular deformations of the equation . . . . . . . . . . . . . . . 847

5 Exact sequences of infinitesimal deformations . . . . . . . . . . . . 853

6 Weakly equisingular and weakly trivial deformations . . . . . . . 857

7 Equipolygonal deformations . . . . . . . . . . . . . . . . . . 860

8 Proofs for weakly equisingular strata . . . . . . . . . . . . 868

9 Geometry of equisingular strata . . . . . . . . . . . . . . 875

References . . . . . . . . . . . . . . . . . . . . 881

Received 6 December 2006, accepted in final form 21 February 2007.

2000 Mathematics Subject Classification 14B07, 14B12, 32S15 (primary), 14H20, 14E15 (secondary).

Keywords: equisingular deformation, plane curve singularities, Milnor number, Hamburger-Noether expansion, deformation of parametrization.

This journal is (c) Foundation Compositio Mathematica 2007. 


\section{A. Campillo, G.-M. Greuel and C. Lossen}

\section{Introduction}

We develop in this paper the theory of equisingular deformations of plane algebroid curve singularities over an algebraically closed field $K$ of arbitrary characteristic. If the curve singularity is given by the equation $f=0$, where $f \in P=K[[x, y]]$ is a reduced formal power series, we study deformations of the local analytic ring $R=P /\langle f\rangle$, as well as deformations of the map $\varphi: P \rightarrow \bar{R}$ where $\bar{R}$ is the integral closure of $R$ in its total ring of fractions. The first are called deformations of the equation and the latter deformations of the parametrization. Since $P$ (respectively, $\bar{R}$ ) are regular local (respectively, semilocal) rings, deformations of the parametrization are very simple objects and the semiuniversal object of the corresponding functor $\underline{\mathcal{D} e} \overline{\bar{R}}_{\bar{R} \leftarrow P}$ of isomorphism classes of deformations of $\varphi: P \rightarrow \bar{P}$ can be explicitly described in terms of a $K$-basis of its tangent space $T_{\bar{R} \leftarrow P}^{1}$. The same holds for deformations with sections $\underline{\mathcal{D} e f} \overline{\mathrm{sec}} \leftarrow P^{\text {. }}$

Equisingular deformations of the parametrization $\varphi$ (with section) are defined by requiring equimultiplicity (along some sections) for each infinitely near point of $P$ on $R$, in a compatible manner. Of course, we have to consider only the finite set of essential infinitely near points occurring in an embedded good resolution of $P /\langle f\rangle$. We prove that the functor $\frac{\mathcal{D} e f}{\bar{R} \leftarrow P}$ of equisingular deformations of the parametrization is a linear subfunctor of $\underline{\mathcal{D} e f} \bar{R} \leftarrow P$ and, therefore, also has an explicit description in terms of a $K$-basis of its tangent space $\overline{T_{\bar{R}}^{1, \mathrm{es}}}$ (Theorem 3.1). In particular, the base space of the semiuniversal deformation of the parametrization is smooth. Furthermore, the linearity allows an easy proof of the openness of versality for equisingular deformations of the parametrization (Corollary 3.8).

The relation between deformations of the parametrization and deformations of the equation is based on the fact that the deformation of $R$ can be uniquely recovered from the deformation of $\varphi: P \rightarrow \bar{R}$. That is, the deformation functor $\underline{\mathcal{D} e f} \overline{\bar{R}}_{\leftarrow}$ is natural isomorphic to the functor $\underline{\operatorname{Def}} \overline{\bar{R} \leftarrow R}$ of deformations of the normalization, that is, of the normalization map $R \rightarrow \bar{R}$. In the same way we get an isomorphism $\underline{\mathcal{D} e f} \overline{\bar{R} \leftarrow P} \cong \underline{\mathcal{D} e f} \overline{\bar{R}} \leftarrow R$ for the corresponding deformations with section (Proposition 1.3) and below we do not distinguish between these two functors. By forgetting $\bar{R}$ we have a natural transformation $\underline{\mathcal{D} e f} \overline{\mathrm{sec}}_{\leftarrow}^{\mathrm{sec}} \rightarrow \underline{\mathcal{D} e f}{ }_{R}^{\mathrm{sec}}$ and we denote the image of $\frac{\mathcal{D} e f}{R \leftarrow P}$ in $\underline{\mathcal{D e f}}_{R}^{\mathrm{sec}}$ by $\frac{\mathcal{D} e f}{R}$ es,sec. We show that equisingular sections of deformations of $R$ are unique (Proposition 2.12) and, hence, by forgetting the section, $\underline{\mathcal{D} e f}{ }_{R}^{\mathrm{es}, \mathrm{sec}}$ is isomorphic to $\underline{\mathcal{D e f}}_{R}^{\mathrm{es}}$. The latter is the functor of (isomorphism classes of) equisingular deformations of the equation (or of $R$ ), which is a subfunctor of $\underline{\mathcal{D} e f}_{R}$, the (usual) deformations of $R$. The transformation $\underline{\mathcal{D} e f} \overline{\mathrm{es}}_{\leftarrow} \leftarrow P \rightarrow \mathcal{D} e f_{R}^{\text {es }}$ from equisingular deformations of the parametrization to equisingular deformations of the equation is, in general, not an isomorphism. However, we show that it is smooth (Theorem 4.2). This implies the first main result, that the base space of the semiuniversal equisingular deformation of $R$ is smooth of dimension equal to the vector space dimension of its tangent space $T_{R}^{1, \mathrm{es}}$, in any characteristic.

We have defined equisingular deformations of $R$ as those which lift to deformations of the parametrization $P \rightarrow \bar{R}$ such that this lifting is equisingular along some sections. While the equisingular sections of deformations of $R$ are unique, the lifting to equisingular sections of deformations of $\bar{R}$ are not, in general, unique in positive characteristic. Indeed, the behaviour of equisingular deformations of the equation depend, in contrast to equisingular deformations of the parametrization, very much on the characteristic $p$ of the field $K$. We say that the characteristic is good (with respect to $R$ ) if $p=0$ or if $p>0$ and $p$ does not divide the multiplicity of any branch of $R$. We prove

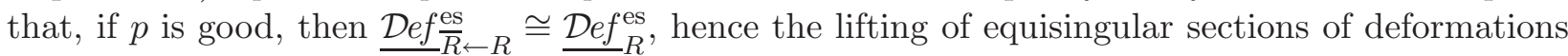
of $R$ to those of deformations of $\bar{R}$ is unique up to isomorphism (Theorem 4.2). Moreover, in this case we can show that the base space of the semiuniversal equisingular deformation of $R$ can be represented by a (smooth) algebraic subscheme of the (algebraic) base space of the semiuniversal deformation of $R$. 


\section{EQUISINGULAR DEFORMATIONS IN ARBITRARY CHARACTERISTIC}

The theory of equisingular deformations of plane curve singularities in characteristic zero has been initiated by Wahl in his thesis (cf. [Wah74a]). Wahl's approach is different from ours as he considers only deformations of the equation and defines equisingularity by requiring equimultiplicity of the equation of the reduced total transform along sections through all essential infinitely near points of $P$ on $R$. Although equimultiplicity of the parametrization is usually stronger than equimultiplicity of the equation, one can prove that Wahl's functor $\overline{\mathrm{ES}}$ and our functor $\underline{D e f}_{R}^{\mathrm{es}}$ are isomorphic (cf. [GLS07]). Thus, we get, in characteristic zero, a new proof of Wahl's result that the equisingularity stratum (which coincides then with the $\mu$-constant stratum, where $\mu$ is the Milnor number) in the base space of the semiuniversal deformation of $R$ is smooth. As mentioned above, the same result holds if the characteristic is good.

Our approach through deformations of the parametrization appears to be quite simple and provides, even in characteristic zero, additional information. This can be seen clearly in $\S 5$ of this paper, where we relate several infinitesimal deformations by means of exact sequences which allow us to compute not only $T_{R}^{1, \text { es }}$ effectively but also gives, on the tangent level, a geometric interpretation of the related deformation functors.

The construction of $\frac{\mathcal{D} e f}{R}$ es as subfunctor of $\frac{\mathcal{D} e f}{R}$ is so explicit that it leads to an algorithm for the computation of a semiuniversal equisingular deformation in good characteristic (that is, of the $\mu$-constant stratum in characteristic zero). This has been implemented in the computer algebra system SingUlAR (cf. [GL07] for a description of the algorithm).

In bad characteristic, however, new phenomena occur. There are deformations which are not equisingular but become equisingular after some finite (and dominant) base change. We call such deformations weakly equisingular and show that the functor of weakly equisingular deformations of $R$ has a semiuniversal object. Its base space is not, in general, smooth but it becomes smooth after a finite and purely inseparable base extension. The proof of this fact is rather involved and occupies $\S \S 7$ and 8 . We prove this by constructing a weak equisingularity stratum in the base space of any deformation of $R$ (with section) which has a certain universal property (Theorems 6.2 and 6.7).

To prove the existence and properties of the weak equisingularity stratum, we give explicit conditions defining a subscheme in the base space of the given deformation such that the restriction of the family to this subscheme can be simultaneously blown up and satisfies additional conditions preserved under further blowing ups. All conditions together define then the weak equisingularity stratum.

We would like to stress that keeping the multiplicity constant along a section in each blowing up is equivalent to keeping the Newton diagram (with respect to generic adapted coordinates) constant. Moreover, we have to consider an adapted Jacobian ideal taking care of the fact that leading terms of $f$ may vanish after differentiation of $f$. This leads to deformations which we call equipolygonal deformations and which we study in detail.

If we start with a versal deformation of $R$ with smooth base space, then the defining conditions for the weak equisingularity stratum become smooth after a purely inseparable base change and we construct the weak equisingularity stratum together with its smooth covering space at the same time. This construction is functorial and, which is a key point, versality for equipolygonal deformations is preserved under blowing ups.

Although the weak equisingularity stratum is not smooth, it is reduced and irreducible, becomes smooth after a purely inseparable base extension and, has good functorial properties. In good characteristic it is even smooth and coincides with the (strong) equisingularity stratum considered above and, therefore, weak and strong equisingular deformations are the same in that case.

In bad characteristic, however, a largest 'strong equisingularity stratum' does not exist in general. Indeed, we show that inside the base space of the semiuniversal deformation of $R$ there may 


\section{A. Campillo, G.-M. Greuel and C. Lossen}

be (infinitely many) different smooth subschemes, all having the same tangent space, such the restriction of the given semiuniversal deformation to them is equisingular (even semiuniversal equisingular). Each of these smooth substrata may be considered as a strong equisingularity stratum, but the restriction of the semiuniversal deformation to the union of two such strata is not (strongly) equisingular. Moreover, the Zariski closure of all of these strong equisingularity strata is the weak equisingularity stratum which is then of bigger dimension.

In the last section we study the geometry of the different equisingularity strata. We identify, inside the smooth covering space of the weak equisingularity stratum, an intrinsically defined subspace $T_{\bar{R} / R}^{1, \text { sec }}$, being the tangent space to deformations of the normalization $R \rightarrow \bar{R}$ which leave $R$ fixed. This space can explicitly be computed, it is zero in good characteristic, and in bad characteristic its vanishing gives a sufficient and necessary condition that a largest (strong) equisingularity stratum exists, and then automatically coincides with the weak equisingularity stratum.

\section{Deformations of the parametrization and of the normalization}

In this section we fix the notation and state some basic facts about deformations of the normalization and deformations of the parametrization for a reduced plane curve singularity. We shall put special emphasis on deformations with section.

By $K$, we denote an algebraically closed field of characteristic $p \geqslant 0$. If $A$ is a Noetherian complete local $K$-algebra with maximal ideal $\mathfrak{m}_{A}$, we always assume that $A / \mathfrak{m}_{A}=K$. The category of these algebras is denoted by $\mathscr{A}_{K}$. We denote the two-dimensional $K$-algebra with $\varepsilon^{2}=0$ by $K[\varepsilon]$.

We consider reduced algebroid plane curve singularities $C$ over $K$, defined by a formal power series $f \in K[[x, y]]$. Usually, we work with the complete local $\operatorname{ring}$ of $C=\operatorname{Spec}(R)$,

$$
R=P /\langle f\rangle, \quad P=K[[x, y]] .
$$

If $f=f_{1} \cdots f_{r}$ is an irreducible factorization of $f$ in $P$, the rings

$$
R_{i}=P /\left\langle f_{i}\right\rangle, \quad i=1, \ldots, r,
$$

are the complete local rings of the branches of $C$. The lowest degree $\operatorname{ord}_{x, y}(f)$ of a monomial appearing in the power series development of $f \neq 0$ is called the multiplicity of $f$ and denoted by $\operatorname{mt}(f)$; we set $\operatorname{mt}(0)=\infty$. Of course, $\operatorname{mt}(f)=\operatorname{mt}\left(f_{1}\right)+\cdots+\operatorname{mt}\left(f_{r}\right)$. We say that the characteristic of $K$ is good (for $R$ ) if it does not divide $\operatorname{mt}\left(f_{i}\right)$, for all $i=1, \ldots, r$.

The normalization $\bar{R}$ of $R$ is the integral closure of $R$ in its total ring of fractions Quot $(R) ; \bar{R}$ is the direct sum of the normalizations $\bar{R}_{i}$ of $R_{i}, i=1, \ldots, r$, hence a semilocal ring. Each $\bar{R}_{i}$ is a discrete valuation ring, and we can choose uniformizing parameters $t_{i}$ such that $\bar{R}_{i} \cong K\left[\left[t_{i}\right]\right]$. After fixing the parameters $t_{i}$, we identify $\bar{R}_{i}$ with $K\left[\left[t_{i}\right]\right]$ and get

$$
\bar{R}=\bigoplus_{i=1}^{r} \bar{R}_{i}=\bigoplus_{i=1}^{r} K\left[\left[t_{i}\right]\right] .
$$

The normalization map $\nu: R \rightarrow \bar{R}$ (induced by the inclusion $R \hookrightarrow$ Quot $(R)$ ) is then given by the (primitive) parametrization of $R$ (or of $C$ ),

$$
\varphi=\left(\varphi_{1}, \ldots, \varphi_{r}\right): P \longrightarrow \bar{R}=\bigoplus_{i=1}^{r} K\left[\left[t_{i}\right]\right],
$$

where $\varphi_{i}(x)=x_{i}\left(t_{i}\right), \varphi_{i}(y)=y_{i}\left(t_{i}\right) \in K\left[\left[t_{i}\right]\right], i=1, \ldots, r$. Since $\langle f\rangle=\operatorname{ker}(\varphi), R$ may be recovered from $\varphi$. We call

$$
\operatorname{ord} \varphi_{i}:=\min \left\{\operatorname{ord}_{t_{i}} x_{i}, \operatorname{ord}_{t_{i}} y_{i}\right\}
$$




\section{EQUISINGULAR DEFORMATIONS IN ARBITRARY CHARACTERISTIC}

the multiplicity (or order) of $\varphi_{i}$ and the $r$-tuple $\operatorname{ord}(\varphi)=\left(\operatorname{ord} \varphi_{1}, \ldots, \operatorname{ord} \varphi_{r}\right)$ the multiplicity (or order) of the parametrization $\varphi$. Note that ord $\varphi_{i}$ is the maximal integer $m_{i}$ such that $\varphi_{i}\left(\mathfrak{m}_{P}\right) \subset$ $\left\langle t_{i}\right\rangle^{m_{i}}$. Moreover, we have (cf. [Cam80])

$$
m t(f)=\operatorname{ord} \varphi_{1}+\cdots+\operatorname{ord} \varphi_{r} .
$$

Definition 1.1. A deformation with sections of the parametrization of $R$ over $A \in \mathscr{A}_{K}$ is a commutative diagram with Cartesian squares

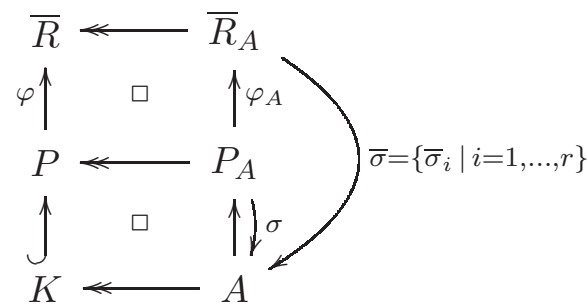

with $\bar{R}_{A}=\bigoplus_{i=1}^{r} \bar{R}_{A, i}$, where $\bar{R}_{A, i}, i=1, \ldots, r$, and $P_{A}$ are Noetherian complete local $K$-algebras which are flat over $A$. Here $\sigma$ is a section of $A \rightarrow P_{A}$, and $\bar{\sigma}_{i}$ is a section of $A \rightarrow \bar{R}_{A, i}, i=1, \ldots, r$. We denote such a deformation by $\xi=\left(\varphi_{A}, \bar{\sigma}, \sigma\right)$.

A morphism from $\xi$ to another deformation $\left(P_{B} \stackrel{\varphi_{B}}{\longrightarrow} \bar{R}_{B}, \bar{\sigma}_{B}, \sigma_{B}\right)$ over $B \in \mathscr{A}_{K}$ is then given by morphisms of local $K$-algebras $A \rightarrow B, P_{A} \rightarrow P_{B}$ and $\bar{R}_{A, i} \rightarrow \bar{R}_{B, i}$ such that the resulting diagram commutes. The category of such deformations is denoted by $\mathcal{D} e f \frac{\mathrm{sec}}{R \leftarrow P}$. If we consider only

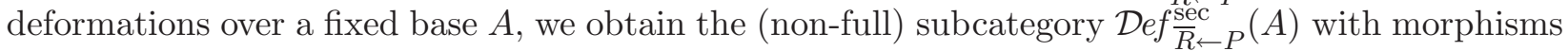
being the identity on $A$. The category $\mathcal{D} e f \frac{\mathrm{sec}}{\bar{R} \leftarrow P}$ is a fibred gruppoid over $\mathscr{A}_{K}$, in particular, each morphism in $\mathcal{D} e f \frac{\mathrm{sec}}{R} \leftarrow P(A)$ is an isomorphism.

Giving $\xi=\left(\varphi_{A}, \bar{\sigma}, \sigma\right)$ and a morphism $\psi: A \rightarrow B$ in $\mathscr{A}_{K}$, the induced deformation $\psi \xi=$ $\left(\psi \varphi_{A}, \psi \bar{\sigma}, \psi \sigma\right)$ is an object in $\operatorname{Def} \frac{\mathrm{sec}}{\bar{R} \leftarrow P}(B)$, defined by $B \rightarrow P_{A} \widehat{\otimes}_{A} B \rightarrow \bar{R}_{A} \widehat{\otimes}_{A} B$, with morphisms $b \mapsto 1 \widehat{\otimes} b, \psi \varphi_{A}=\varphi_{A} \widehat{\otimes} \operatorname{id}_{B}, \psi \sigma: h \widehat{\otimes} b \mapsto \psi(\sigma(h)) \cdot b, \psi \bar{\sigma}_{i}: r \widehat{\otimes} b \mapsto \psi\left(\bar{\sigma}_{i}(r)\right) \cdot b$. Here, $\widehat{\otimes}$ denotes the complete tensor product.

The set of isomorphism classes of objects in $\mathcal{D} e f \frac{\mathrm{sec}}{\bar{R} \leftarrow P}(A)$ is denoted by $\underline{\mathcal{D} e f} \frac{\mathrm{sec}}{\bar{R} \leftarrow P}(A)$, and $\underline{\mathcal{D} e f} \frac{\mathrm{sec}}{\bar{R} \leftarrow P}$ : $\mathscr{A}_{K} \rightarrow$ (Sets) denotes the corresponding deformation functor (which always refers to isomorphism classes). Moreover, we denote by $T_{\bar{R} \leftarrow P}^{1, \text { sec }}:=\underline{\operatorname{Def}} \frac{\sec }{\bar{R} \leftarrow P}(K[\varepsilon])$ the tangent space to this functor.

Remark 1.2. Since $P$ and the $\bar{R}_{i}$ are regular local rings, any deformation of $P$ and of $\bar{R}$ is trivial. That is, there are isomorphisms $P_{A} \cong A[[x, y]]$ and $\bar{R}_{A} \cong \bigoplus_{i=1}^{r} A\left[\left[t_{i}\right]\right]$ over $A$, mapping the sections $\sigma$ and $\bar{\sigma}_{i}$ to the trivial sections. Hence, any object in $\mathcal{D e f} \frac{\mathrm{sec}}{R \leftarrow P}(A)$ is isomorphic to a diagram of the form

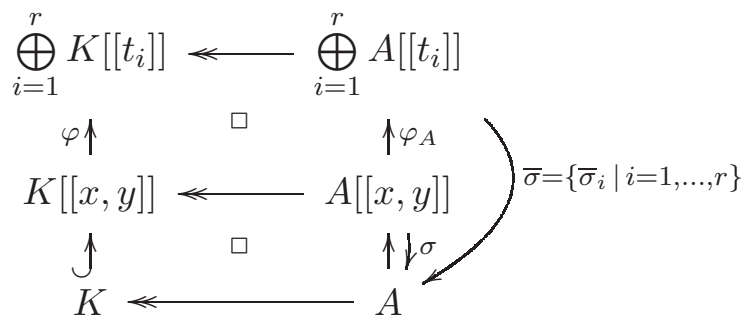

where $\varphi_{A}$ is the identity on $A$ and $\sigma, \bar{\sigma}_{i}$ are the trivial sections (that is, the canonical epimorphisms $\bmod x, y$, respectively $\left.\bmod t_{i}\right)$. Hence, $\varphi_{A}$ is given by $\varphi_{A}=\left(\varphi_{A, 1}, \ldots, \varphi_{A, r}\right)$, where $\varphi_{A, i}$ is determined by

$$
\varphi_{A, i}(x)=X_{i}\left(t_{i}\right), \quad \varphi_{A, i}(y)=Y_{i}\left(t_{i}\right) \in t_{i} A\left[\left[t_{i}\right]\right],
$$

$i=1, \ldots, r$, such that $X_{i}\left(t_{i}\right) \equiv x_{i}\left(t_{i}\right), Y_{i}\left(t_{i}\right) \equiv y_{i}\left(t_{i}\right) \bmod \mathfrak{m}_{A}$. 


\section{A. Campillo, G.-M. Greuel and C. Lossen}

Similarly to Definition 1.1, by replacing $P$ by $R$ and $\varphi$ by $\nu$ (respectively, $P_{A}$ by $R_{A}$ and $\varphi_{A}$ by $\nu_{A}$ ), we can define deformations with section of the normalization $R \rightarrow \bar{R}$, and obtain the category

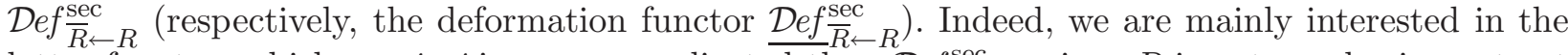
latter functor, which a priori is more complicated than $\underline{\mathcal{D} e f} \frac{\sec }{R} \leftarrow P$, since $R$ is not regular in contrast to $P$. The following proposition shows that both functors are isomorphic. To prove this, we have to consider deformations with section of the sequence of morphisms $P \rightarrow R \rightarrow \bar{R}$, whose definition is analogous to Definition 1.1. Such deformations may be called deformations of the normalization with embedding. The corresponding deformation category (respectively, the corresponding deformation functor of isomorphism classes) is denoted by $\mathcal{D} e f \frac{\mathrm{sec}}{R \leftarrow R \leftarrow P}\left(\right.$ respectively, by $\left.\underline{\mathcal{D} e f} \frac{\mathrm{sec}}{\bar{R} \leftarrow R \leftarrow P}\right)$.

Proposition 1.3. The forgetful functor from $\mathcal{D}$ ef $\frac{\sec }{\bar{R} \leftarrow R \leftarrow P}(A)$ to $\operatorname{Def} \frac{\sec }{R \leftarrow P}(A)$ (respectively, to $\left.\operatorname{Def} \frac{\mathrm{sec}}{R \leftarrow R}(A)\right)$ is an isomorphism (respectively, smooth). Both induce an isomorphism between the corresponding deformation functors. In particular,

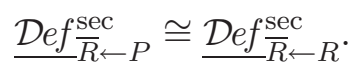

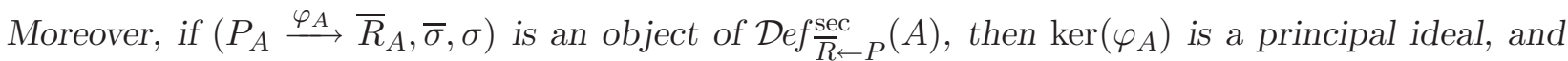
the lifting of $\left(\varphi_{A}, \bar{\sigma}, \sigma\right)$ to an object of $\mathcal{D} e f \frac{\sec }{R \leftarrow R \leftarrow P}(A)$ is obtained by setting $R_{A}=P_{A} / \operatorname{ker}\left(\varphi_{A}\right)$.

Proof. Let $\left(P_{A} \stackrel{\varphi_{A}}{\longrightarrow} \bar{R}_{A}, \bar{\sigma}, \sigma\right)$ be a deformation with sections of the parametrization $P \rightarrow \bar{R}$ over $A \in \mathscr{A}_{K}$. Since $\varphi_{A}$ is quasifinite, $\bar{R}_{A}$ is a finite $P_{A}$-module, and we have a minimal free resolution

$$
0 \longleftarrow \bar{R}_{A} \longleftarrow F_{0} \stackrel{M}{\longleftarrow} F_{1} \longleftarrow F_{2} \longleftarrow \cdots
$$

of $\bar{R}_{A}$ as a $P_{A}$-module. Since $P_{A}$ and $\bar{R}_{A}$ are $A$-flat, the exactness of the sequence (1.1) is preserved when tensoring with $\otimes_{A} K$, obtaining in this way a minimal free resolution of $\bar{R}$ as $P$-module with presentation matrix $M_{0}=M \otimes_{A} K$. Since $P$ is regular of dimension two, and since $\bar{R}$ has depth one, the Auslander-Buchsbaum formula gives that the minimal resolution of $\bar{R}$ has length one. Thus, $M_{0}$ is injective. By the local criterion of flatness (cf. [Mat86, Theorem 22.5]), $M$ is injective too, and the free $P_{A}$-modules $F_{0}$ and $F_{1}$ have the same rank.

The ideal $\langle\operatorname{det}(M)\rangle \subset P_{A}$ is independent of the chosen resolution, and we set $R_{A}:=P_{A} /$ $\langle\operatorname{det}(M)\rangle$, which is flat over $A$. Note that the ideals $\langle f\rangle$ and $\left\langle\operatorname{det}\left(M_{0}\right)\right\rangle$ of $P$ have the same support and coincide in the generic points where $R=P /\langle f\rangle$ is regular. It follows that the two principal ideals $\langle f\rangle$ and $\left\langle\operatorname{det}\left(M_{0}\right)\right\rangle$ of $P$ coincide.

Since $\operatorname{det}(M)$ annihilates $\bar{R}_{A}$ by Cramer's rule, and since the kernel of $\varphi_{A}$ is equal to the annihilator of $\bar{R}_{A}$ as $P_{A}$-module, the canonical projection $P_{A} \rightarrow R_{A}^{\prime}:=P_{A} / \operatorname{ker} \varphi_{A}$ induces a surjection $R_{A} \rightarrow R_{A}^{\prime}$. The kernel of this surjection is supported by the singular locus of the fibres and is zero after tensoring with $\otimes_{A} K$. Thus, by Nakayama's lemma, $R_{A}=R_{A}^{\prime}$. It follows that $\varphi_{A}$ factors as $P_{A} \rightarrow R_{A} \hookrightarrow \bar{R}_{A}$, defining in this way an object of $\mathcal{D} e f \frac{\mathrm{sec}}{R \leftarrow R \leftarrow P}(A)$. Moreover, if $P_{A} \rightarrow R_{A}^{\prime \prime} \hookrightarrow \bar{R}_{A}$ is any lifting of $P_{A} \rightarrow \bar{R}_{A}$ to an object of $\mathcal{D e f} \overline{\mathrm{sec}}_{\leftarrow \leftarrow P}(A)$, then $P_{A} \rightarrow R_{A}^{\prime \prime}$ is surjective and $R_{A}^{\prime \prime} \hookrightarrow \bar{R}_{A}$ is injective (by Nakayama's lemma). Thus, as before, $R_{A}^{\prime \prime}=R_{A}$. As

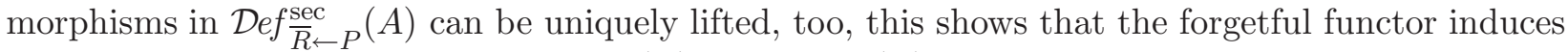
an equivalence of categories $\mathcal{D} e f \frac{\sec }{R \leftarrow R \leftarrow P}(A) \cong \mathcal{D} e f \frac{\sec }{R \leftarrow P}(A)$.

To get the statements for deformations (with section) of the normalization, note that $P$ is a regular local ring. Hence, any deformation (with section) of $R$ may be lifted to an 'embedded' deformation, that is, a deformation of $P \rightarrow R$. Thus, the forgetful functor induces a surjection $\mathcal{D} e f \frac{\mathrm{sec}}{\bar{R} \leftarrow R \leftarrow P}(A) \rightarrow \mathcal{D} e f \frac{\mathrm{sec}}{\bar{R} \leftarrow R}(A)$. The fibre is a principal homogeneous space under isomorphisms of $P$ fixing $R$, showing smoothness. Moreover, if two deformations $R_{A}$ and $R_{A}^{\prime}$ of $R$ over $A$ are isomorphic, the isomorphism $R_{A} \cong R_{A}^{\prime}$ may be lifted to an isomorphism $P_{A} \cong P_{A}^{\prime}$ (since $P_{A} \cong A[[x, y]]$ ).

Remark 1.4. If we omit the sections in Definition 1.1 and in the subsequent discussion, we get analogous results for deformations without sections. The corresponding categories (respectively 


\section{EQUISINGULAR DEFORMATIONS IN ARBITRARY CHARACTERISTIC}

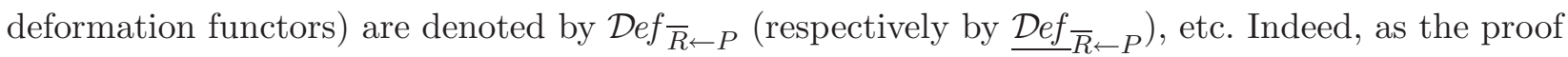
of Proposition 1.3 shows, the sections do not affect the arguments at all. Hence, Proposition 1.3 remains true with the superscript 'sec' being omitted.

\section{Equisingular deformations of the parametrization}

In this section, we define equisingular deformations of the parametrization with section, and we discuss the uniqueness of the sections.

In order to define equisingular deformations, we consider the notion of infinitely near points.

Consider the natural diagram of graded $K$-algebras given by

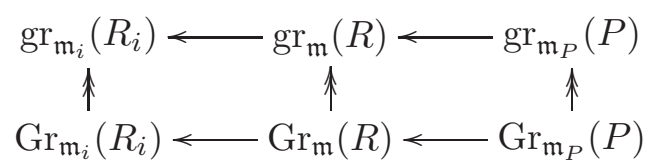

where $\mathfrak{m}, \mathfrak{m}_{i}, \mathfrak{m}_{P}$ are the respective maximal ideals of $R, R_{i}, P$ and where, for an ideal $I \subset S$, $\operatorname{gr}_{I}(S)=\bigoplus_{k=0}^{\infty} I^{k} / I^{k+1}, \operatorname{Gr}_{I}(S)=\bigoplus_{k=0}^{\infty} I^{k}$. Applying Proj to the above diagram, we get the blowup schemes $\mathrm{B} \ell_{\mathfrak{m}_{i}}\left(R_{i}\right), \mathrm{B} \ell_{\mathfrak{m}}(R)$, and $\mathrm{B} \ell_{\mathfrak{m}_{P}}(P)$ in the lower row, and the corresponding exceptional divisors $E_{\mathfrak{m}_{i}}, E_{\mathfrak{m}}$, and $E_{P}$ in the upper row. Applying Proj to the vertical maps in the diagram gives rise to natural embeddings of these objects.

Definition 2.1. An infinitely near point $P^{\prime}$ in the first infinitesimal neighbourhood of $P$ is the completion of the local ring of a closed point $O$ on the exceptional divisor $E_{P}$ in $\mathrm{B} \ell_{\mathfrak{m}_{P}}(P)$. We always use the same notation $O$ for the local ring and for the point in $\mathrm{B} \ell_{\mathfrak{m}_{P}}(P)$.

Since exceptional divisors are projectivizations of tangent cones, $E_{P}$ is a projective line, and the image of $E_{\mathfrak{m}_{i}}$ in $E_{P}$ is one point $O_{i}$, counted $m_{i}$ times, where $m_{i}$ is the multiplicity of the branch $R_{i}$. Among the infinitely near points in the first neighbourhood, those of type $P^{\prime}=\widehat{O}_{i}$ for some $1 \leqslant i \leqslant r$ are called infinitely near points (of $P$ ) on $R$. For such a $P^{\prime}$, we set

$$
\Lambda_{P^{\prime}}:=\left\{i \in\{1, \ldots, r\} \mid P^{\prime}=\widehat{O}_{i}\right\} .
$$

For each $i \in \Lambda_{P^{\prime}}$, we also say that the branch $R_{i}$ passes through $P^{\prime}$.

Note that we refer to $P$ itself as an infinitely near point of $P$ (in the zeroth infinitesimal neighbourhood) on $R$.

Remark 2.2. In analytical terms, we have $P=K[[x, y]], \operatorname{gr}_{\mathfrak{m}_{P}}(P)=K[x, y], \operatorname{gr}_{\mathfrak{m}}(R)=K[x, y] /$ $\left\langle J_{m} f\right\rangle$, and $\operatorname{gr}_{\mathfrak{m}_{i}}\left(R_{i}\right)=K[x, y] /\left\langle J_{m_{i}} f_{i}\right\rangle, i=1, \ldots, r$, where $J_{m_{i}} f_{i}$ denotes the sum of terms of smallest degree $m_{i}=\operatorname{mt}\left(f_{i}\right)$ in the power series expansion of $f_{i}$, and $J_{m} f=\prod_{i=1}^{r} J_{m_{i}} f_{i}$.

Note that each $J_{m_{i}} f_{i}$ is the $m_{i}$ th power of a non-zero linear form $\alpha_{i} y-\beta_{i} x, \alpha_{i}, \beta_{i} \in K$. We also say that it corresponds to the tangent direction $\left(\alpha_{i}: \beta_{i}\right) \in \mathbb{P}_{K}^{1}$. If the infinitely near point $O \in E_{P}$ corresponds to the tangent direction $(1: \beta) \in \mathbb{P}_{K}^{1}$, then $O=P[y / x]_{\left\langle x, y^{\prime}\right\rangle}, y^{\prime}=y / x-\beta$, and $P^{\prime}=\widehat{O}=K\left[\left[x, y^{\prime}\right]\right]$.

Let $\pi^{\prime}: P \rightarrow P^{\prime}$ be the blow-up map $x \mapsto x, y \mapsto x\left(y^{\prime}+\beta\right)$. For $g \in P$ (of multiplicity $m$ ) such that $J_{m} g=c(y-\beta x)^{m}$ for some $c \in K^{*}$ we set

$$
g^{\prime}=x^{-m} \pi^{\prime}(g), \quad \widehat{g}=x g^{\prime}
$$

and call $R^{\prime}=P^{\prime} /\left\langle g^{\prime}\right\rangle$ the strict transform (respectively, $\widehat{R}=P^{\prime} /\langle\widehat{g}\rangle$ the reduced total transform) of $R=P /\langle g\rangle$. Moreover, $P^{\prime} /\left\langle\pi^{\prime}(g)\right\rangle$ (respectively, $P^{\prime} /\langle x\rangle$ ) is the total transform of $R$ (respectively, the exceptional curve of the blow-up). In particular, if $O=O_{i}$ (that is, $\alpha_{i} \neq 0$ and $\beta=\beta_{i} / \alpha_{i}$ ), then $R_{i}^{\prime}=P^{\prime} /\left\langle f_{i}^{\prime}\right\rangle$ (respectively $R^{\prime}=P^{\prime} /\left\langle\prod_{i \in \Lambda_{P^{\prime}}} f_{i}^{\prime}\right\rangle$ ) is the strict transform of the branch $R_{i}$ (respectively of $R$ ), at $P^{\prime}$. 


\section{A. Campillo, G.-M. Greuel and C. Lossen}

For each infinitely near point $P^{\prime}$ in the first infinitesimal neighbourhood on $R$, and for each $i \in \Lambda_{P^{\prime}}$, the normalization of the strict transform $R_{i}^{\prime}$ is $\bar{R}_{i}$, and the normalization of $R^{\prime}$ is $\bar{R}:=$ $\bigoplus_{i \in \Lambda_{P^{\prime}}} \bar{R}_{i}$. Further, note that the parametrization $P^{\prime} \rightarrow \bar{R}^{\prime}$ of $R^{\prime}$ is given by $x_{i}\left(t_{i}\right), y_{i}^{\prime}\left(t_{i}\right), i \in \Lambda_{P^{\prime}}$, where

$$
y_{i}^{\prime}\left(t_{i}\right):=\frac{y_{i}\left(t_{i}\right)}{x_{i}\left(t_{i}\right)}-\frac{\beta_{i}}{\alpha_{i}} \in K\left[\left[t_{i}\right]\right] .
$$

Here again, we assume that $\alpha_{i} \neq 0$ (which is no restriction, since after a general linear change of the coordinates $x, y$ it holds for all $i=1, \ldots, r)$.

We extend the above definitions to higher infinitesimal neighbourhoods by induction.

Definition 2.3. Let $k \geqslant 2$, and assume that the infinitely near points $P^{\prime}$ on $R$ in the $(k-1)$ th neighbourhood of $P$ are defined. Assume also that for each of these points $P^{\prime}$ a set $\Lambda_{P^{\prime}}$, the strict transform $R_{i}^{\prime}$ of each branch $R_{i}, i \in \Lambda_{P^{\prime}}$, and the strict transform $R^{\prime}$ of $R$ at $P$ are defined, as well as the reduced total transform $\widehat{R}_{i}^{\prime}$ respectively, $\widehat{R^{\prime}}$ and the exceptional curve $E^{\prime}$. Then, we call each infinitely near point $P^{\prime \prime}$ on $R$ in the first infinitesimal neighbourhood of such a point $P^{\prime}$ an infinitely near point on $R$ in the $k$ th neighbourhood of $P$. We introduce

$$
\Lambda_{P^{\prime \prime}}:=\left\{i \in \Lambda_{P^{\prime}} \mid R_{i}^{\prime} \text { passes through } P^{\prime \prime}\right\}
$$

and define the strict transform of $R_{i}$ (respectively, $R$ ) at $P^{\prime \prime}$ to be the strict transform of $R_{i}^{\prime}$ (respectively, $R^{\prime}$ ) at $P^{\prime}$. Moreover, the reduced total transform of $R_{i}$ (respectively, $R$ ) at $P^{\prime \prime}$ is the reduced total transform of $\widehat{R_{i}^{\prime}}$ (respectively, $\widehat{R^{\prime}}$ ) and the exceptional curve at $P^{\prime \prime}$ is the reduced total transform of $E^{\prime}$.

Given infinitely near points $P^{\prime}, P^{\prime \prime}$ as above, we call $P^{\prime \prime}$ consecutive to $P^{\prime}$. According to Remark 2.2, if $P^{\prime}=K[[u, v]], P^{\prime \prime}=K[[w, z]]$, then up to interchanging $u$ and $v$, we can assume that $w=u, z=v / u-\beta$ for some $\beta \in K$. The map $P^{\prime} \rightarrow P^{\prime \prime}$ is called a formal blow-up (of the maximal ideal $\mathfrak{m}_{P^{\prime}}$ in $P^{\prime}$ ), as it satisfies the following two properties:

(i) $\mathfrak{m}_{P^{\prime}} P^{\prime \prime}=\langle u, v\rangle \cdot P^{\prime \prime}$ is a principal ideal; and

(ii) there is no proper subalgebra $S \in \mathscr{A}_{K}$ of $P^{\prime \prime}$ with $\mathfrak{m}_{P^{\prime}} S$ being a principal ideal.

We call a point $P^{\prime}$ an infinitely near point of $P$ on $R$ if it is an infinitely near point on $R$ in the $k$ th neighbourhood of $P$ for some $k \geqslant 0$. The above consideration shows that infinitely near points of $P$ on $R$ are related to $P$ by compositions of formal blow-ups. An infinitely near point $P^{\prime}$ of $R$ is called free (respectively, satellite) if exactly one (respectively, two) components of the exceptional curve $E^{\prime}$ pass through $P^{\prime}$. The point $P$ itself is considered as free.

We say that an infinitely near point $P^{\prime} \neq P$ is essential for $R$ if the reduced total transform of $R$ at $P^{\prime}$ is not a node (i.e. a normal crossing of two smooth branches). If $R$ is not smooth $P$ itself is essential. The set of essential points for $R$ is denoted by $\operatorname{Ess}(R)$. The set $\operatorname{Ess}(R)$ will be considered for an embedded (good) resolution of $R$. By the theorem of resolution of singularities (cf, e.g. [Cam80, Lip88, Zar38]), $\operatorname{Ess}(R)$ is finite.

DeFINITION 2.4. We define the multiplicity (or order) of a deformation with sections of the parametrization $\left(\varphi_{A}, \bar{\sigma}, \sigma\right)$, to be the $r$-tuple $\operatorname{ord}\left(\varphi_{A} \bar{\sigma}, \sigma\right):=\mathbf{m}=\left(m_{1}, \ldots, m_{r}\right)$ such that $\varphi_{A, i}\left(I_{\sigma}\right) \subset$ $I_{\bar{\sigma}_{i}}^{m_{i}}$ and $m_{i}$ is the maximal integer with this property. Here, $I_{\sigma}=\operatorname{ker} \sigma \subset P_{A}$ and $I_{\bar{\sigma}_{i}}=\operatorname{ker} \bar{\sigma}_{i} \subset \bar{R}_{A, i}$ are the ideals of the sections. A deformation with sections $\left(\varphi_{A}, \bar{\sigma}, \sigma\right)$ of $\varphi$ is called equimultiple (or an em-deformation) if

$$
\operatorname{ord}\left(\varphi_{A}, \bar{\sigma}, \sigma\right)=\operatorname{ord}(\varphi)
$$

We introduce the category (respectively, deformation functor) of em-deformations with sections of the parametrization, $\mathcal{D} e f \frac{\mathrm{em}}{\bar{R} \leftarrow P}$ (respectively, $\left.\underline{\mathcal{D} e f} \frac{e m}{\bar{R} \leftarrow P}\right)$. 


\section{EQUISINGULAR DEFORMATIONS IN ARBITRARY CHARACTERISTIC}

Remark 2.5. A deformation $\varphi_{A}: A[[x, y]] \rightarrow \bigoplus_{i=1}^{r} A\left[\left[t_{i}\right]\right]$ of the parametrization with trivial sections as in Remark 1.2, given by power series $X_{i}\left(t_{i}\right), Y_{i}\left(t_{i}\right) \in A\left[\left[t_{i}\right]\right]$ is equimultiple if and only if, for each $1 \leqslant i \leqslant r$, the minimum of $\operatorname{ord}_{t_{i}} X_{i}$ and $\operatorname{ord}_{t_{i}} Y_{i}$ coincides with the minimum of the $t_{i}$-orders of the residues $x_{i}, y_{i} \bmod \mathfrak{m}_{A}$. If this minimum is attained by, say $x_{i}\left(t_{i}\right)$, this means that the coefficient of the term of smallest $t_{i}$-degree in $X_{i}$ is a unit in $A$. If the deformation with trivial sections defined by $\varphi_{A}$ is equimultiple, then each generator $F \in A[[x, y]]$ of $\operatorname{ker} \varphi_{A}$ (which is a principal ideal due to Proposition 1.3) defines an em-deformation of $R=K[[x, y]] /\langle f\rangle$, i.e. $\operatorname{ord}_{x, y}(F)=\operatorname{ord}_{x, y}(f)$ (cf. [GLS07, Lemma 2.26] for a proof for $k=\mathbb{C}$ which can be modified to work for arbitrary $K$ ).

Notice, however, that the converse is not true if $A$ is not reduced. For instance, consider the irreducible plane curve singularity $R=K[[x, y]] /\left\langle x^{5}+y^{3}\right\rangle$. The deformation of the parametrization (with trivial sections) over $A=K[\varepsilon]$ given by $X(t)=t^{3}-3 \varepsilon t, Y(t)=t^{5}+5 \varepsilon t^{3}$ is obviously not equimultiple as a deformation of the parametrization. However, the corresponding deformation of $R$, which is given by $F=x^{5}+y^{3}$, is trivial, hence equimultiple.

Definition 2.6. (1) An equisingular deformation of the parametrization $P \rightarrow \bar{R}$ (or es-deformation of $P \rightarrow \bar{R})$ over $A$ is a deformation with sections $\left(\varphi_{A}, \bar{\sigma}, \sigma\right)$ of the parametrization which is equimultiple and which satisfies.

For each infinitely near point $P^{\prime}$ on $R$ there exists a deformation $\left(\varphi_{A}^{\prime}, \bar{\sigma}^{\prime}, \sigma^{\prime}\right)$ of the parametrization $P^{\prime} \rightarrow \bar{R}^{\prime}$ over $A$ such that the following diagram is commutative with Cartesian squares

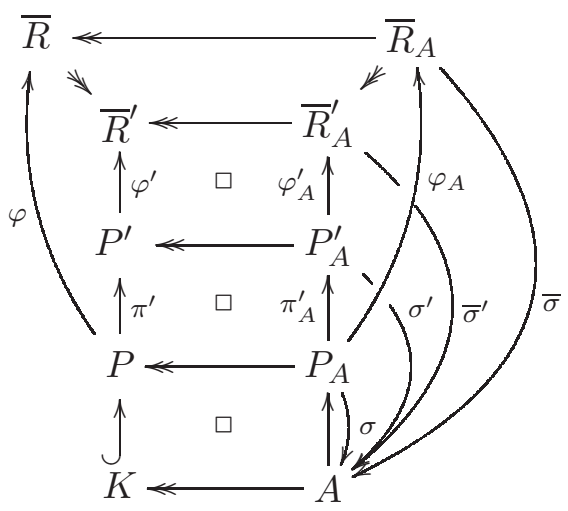

and the following conditions hold:

(i) We have $\bar{\sigma}_{i}^{\prime}=\bar{\sigma}_{i}: \bar{R}_{A, i} \rightarrow A$ for all $i \in \Lambda_{P^{\prime}}$. If $P^{\prime}=P$ then $\left(\varphi_{A}^{\prime}, \bar{\sigma}^{\prime}, \sigma^{\prime}\right)=\left(\varphi_{A}, \bar{\sigma}, \sigma\right)$.

(ii) The deformation $\left(P_{A}^{\prime} \stackrel{\varphi_{A}^{\prime}}{\longrightarrow} \bar{R}_{A}^{\prime}, \bar{\sigma}^{\prime}, \sigma^{\prime}\right)$ is equimultiple, i.e. an object of $\operatorname{Def} \frac{\mathrm{em}}{\bar{R}^{\prime} \leftarrow P^{\prime}}(A)$.

(iii) The system of such diagrams is compatible: that is, if $P^{\prime \prime}$ on $R$ is in some infinitesimal neighbourhood of $P^{\prime}$, then there exists a morphism $P_{A}^{\prime} \rightarrow P_{A}^{\prime \prime}$ such that the obvious diagram commutes.

(iv) If $P^{\prime \prime}$ is consecutive to $P^{\prime}$, then $P_{A}^{\prime} \rightarrow P_{A}^{\prime \prime}$ is a formal blow-up of the section $\sigma^{\prime}: P_{A}^{\prime} \rightarrow A$. That is, $I_{\sigma}^{\prime} P_{A}^{\prime \prime}$ is a principal ideal and $P_{A}^{\prime \prime}$ does not contain a proper complete local $A$-subalgebra $S$ such that $I_{\sigma}^{\prime} S$ is principal.

The corresponding (full) subcategory of $\mathcal{D} e f \frac{\mathrm{sec}}{\bar{R} \leftarrow P}$ is denoted by $\mathcal{D} e f \frac{\mathrm{es}}{R} \leftarrow P$, and the subfunctor of $\frac{\mathcal{D} e f}{\sec } \leftarrow P$ of isomorphism classes of equisingular deformations of the parametrization is denoted by $\underline{\mathcal{D} e f} \bar{R} \leftarrow P$.

(2) An object $\xi=\left(\nu_{A}, \bar{\sigma}, \sigma\right) \in \mathcal{D} \operatorname{se} \frac{\text { sec }}{R}(A)$ is called an equisingular deformation of the nor-

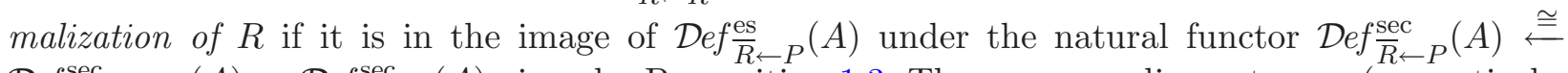
$\mathcal{D} e f \frac{\mathrm{sec}}{\bar{R} \leftarrow R \leftarrow P}(A) \rightarrow \mathcal{D} e f \frac{\mathrm{sec}}{\bar{R} \leftarrow R}(A)$ given by Proposition 1.3. The corresponding category (respectively, deformation) functor is denoted by $\mathcal{D} e f \frac{\mathrm{es}}{R \leftarrow R}\left(\right.$ respectively, $\underline{\mathcal{D} e f} \frac{\mathrm{es}}{R} \leftarrow R$ ). 


\section{A. Campillo, G.-M. Greuel and C. Lossen}

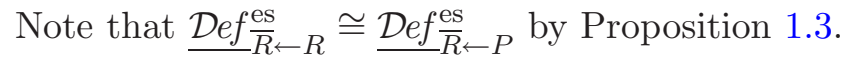

Remark 2.7. (1) Condition (i), together with the fact that $\left(\varphi_{A}^{\prime}, \bar{\sigma}^{\prime}, \sigma^{\prime}\right)$ defines a deformation with section of the parametrization $P^{\prime} \rightarrow \bar{R}^{\prime}$, implies that the composition $P_{A}^{\prime} \rightarrow \bar{R}_{A, i} \stackrel{\bar{\sigma}_{i}}{\longrightarrow} A$ is independent of $i \in \Lambda_{P^{\prime}}$. If $P^{\prime \prime}$ is an infinitely near point of $P^{\prime}$, the image of $I_{\sigma^{\prime}}$ in $P_{A}^{\prime \prime}$ is contained in $I_{\sigma^{\prime \prime}}$.

(2) If $\bar{\sigma}_{i}\left(t_{i}\right)=a \in \mathfrak{m}_{A}$, then replacing $t_{i}$ by $t_{i}-a$ trivializes the section $\bar{\sigma}_{i}$. In the same way, each section $\sigma^{\prime}$ can be trivialized by choosing appropriate generators $u, v$ for $I_{\sigma^{\prime}} \subset P^{\prime}$ (which is possible due to Nakayama's lemma). This choice corresponds to the choice of an isomorphism, $P_{A}^{\prime} \cong A[[u, v]]$ (up to reordering the indeterminates).

(3) Let $P^{\prime \prime}$ be consecutive to $P^{\prime}$. Then the formal blow-up $P_{A}^{\prime} \rightarrow P_{A}^{\prime \prime}$ of the section $\sigma^{\prime}: P_{A}^{\prime} \rightarrow$ $A$ can be expressed in the same way as the formal blow-up of the maximal ideal of a point in Definition 2.3.

According to part (2), we may assume that $P_{A}^{\prime}=A[[u, v]]$ and $P_{A}^{\prime \prime}=A[[w, z]]$ and that the sections $\sigma^{\prime}, \sigma^{\prime \prime}$ are trivial. In addition, we assume that $m(u) \leqslant m(v)$, where $m$ denotes the $\langle w, z\rangle$ order of the residue $\bmod \mathfrak{m}_{A}$ of elements in $A[[z, w]]$. Since $P_{A}^{\prime} \rightarrow P_{A}^{\prime \prime}$ is a formal blow-up, one has $\langle u, v\rangle A[[w, z]]=\langle h\rangle A[[w, z]]$ for some $h \in A[[w, z]]$. Therefore, $u=h p, v=h q$, and $h=r u+s v$ for some $p, q, r, s \in A[[w, z]]$. Hence, $m(u)=m(h)+m(p) \geqslant m(u)+\nu(p)$, which implies $m(p)=0$, that is, $p$ is a unit in $A[[w, z]]$. Replacing $h$ by $h p=u$, we get $v=u\left(q p^{-1}\right)=u\left(v^{\prime}+\beta\right)$ for a unique $v^{\prime} \in\langle w, z\rangle_{A}$ and $\beta \in A$, and thus $\langle u, v\rangle A\left[\left[u, v^{\prime}\right]\right]=\langle u\rangle A\left[\left[u, v^{\prime}\right]\right]$. The minimality condition for the formal blow-up implies now that $A\left[\left[u, v^{\prime}\right]\right]=A[[w, z]]$ and, therefore, $\left\langle u, v^{\prime}\right\rangle=\langle w, z\rangle=I_{\sigma^{\prime \prime}}$. Thus, we may choose $w=u$ and $z=v / u-\beta$, that is, the formal blow-up of $\sigma^{\prime}$ is given by $A[[u, v]] \rightarrow A[[w, z]]$, $u \mapsto w, v \mapsto w(z+\beta)$ for a unique $\beta \in A$.

(4) Although the set of infinitely near points $P^{\prime}$ on $R$ is infinite we need to consider in Definition 2.6 only the subset of $\operatorname{Ess}(R)$ consisting of those $P^{\prime}$ for which the strict transform $R^{\prime}$ of $R$ is singular (which is finite since $\operatorname{Ess}(R)$ is finite).

Before giving a proof for the existence of a semiuniversal deformation for equisingular deformations of the parametrization, we consider (versal) equimultiple deformations.

Recall the notion of versality. A deformation $\xi$ over some base $B$ is versal if the following holds: given two deformations $\widetilde{\eta}$ and $\eta$ over $\widetilde{C}$ and $C$, respectively, such that $\eta$ is induced from $\widetilde{\eta}$ by a surjective morphism $\chi: \widetilde{C} \rightarrow C$ and from $\xi$ by a morphism $\psi: B \rightarrow C$. Then $\widetilde{\eta}$ can be induced from $\xi$ by a morphism $\widetilde{\psi}: B \rightarrow \widetilde{C}$ satisfying $\chi \circ \widetilde{\psi}=\psi$. $\xi$ is called semiuniversal if, moreover, the tangent map of $\widetilde{\psi}$ is uniquely determined.

We shall identify explicitly a semiuniversal deformation for $\underline{\mathcal{D} e f} \frac{\mathrm{es}}{\bar{R} \leftarrow P}$ as a subfamily of a semiuniversal deformation for $\underline{\mathcal{D} e f} \frac{\mathrm{sec}}{\bar{R} \leftarrow P}$.

We begin by describing a semiuniversal deformation for $\underline{\mathcal{D} e f} \overline{\mathrm{sec}} \leftarrow P$. We do this in a slightly more general context: given an integer vector $\mathbf{m}=\left(m_{1}, \ldots, m_{r}\right)$ such that either $\mathbf{m}=\mathbf{0}=(0, \ldots, 0)$, or $1 \leqslant m_{i} \leqslant \operatorname{ord} \varphi_{i}$, we call a deformation of the parametrization over $A,\left(P_{A} \stackrel{\varphi_{A}}{\longrightarrow} \bar{R}_{A}, \bar{\sigma}, \sigma\right)$, an $\mathbf{m}$-multiple deformation if

$$
\varphi_{A}\left(I_{\sigma}\right) \subset I_{\bar{\sigma}}^{\mathbf{m}}:=\bigoplus_{i=1}^{r} I_{\bar{\sigma}_{i}}^{m_{i}} .
$$

Here, $I_{\bar{\sigma}_{i}}:=\operatorname{ker} \bar{\sigma}_{i} \subset \bar{R}_{A, i}$ and $I_{\sigma}=\operatorname{ker} \sigma \subset P_{A}$. The corresponding category is denoted by $\operatorname{Def} \frac{\mathbf{m}}{\bar{R} \leftarrow P}(A)$, and the deformation functor by $\underline{\mathcal{D} e f} \frac{\mathbf{m}}{\bar{R} \leftarrow P}$.

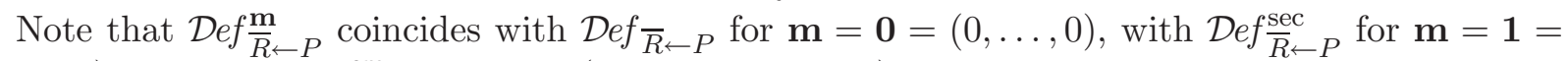
$(1, \ldots, 1)$, and with $\mathcal{D} e f \frac{\mathrm{em}}{R \leftarrow P}$ for $\mathbf{m}=\left(\operatorname{ord} \varphi_{1}, \ldots\right.$, ord $\left.\varphi_{r}\right)$. 


\section{EQUISINGULAR DEFORMATIONS IN ARBITRARY CHARACTERISTIC}

The following proposition gives an explicit description of the tangent space $T_{\bar{R} \leftarrow P}^{1, \mathbf{m}}:=\underline{\operatorname{Def}} \overline{\mathbf{m}} \leftarrow P$ $(K[\varepsilon])$ to the functor of $\mathbf{m}$-multiple deformations of the parametrization. We start by introducing the notation

$$
\dot{\mathbf{x}}:=\left(\begin{array}{c}
\dot{x}_{1} \\
\vdots \\
\dot{x}_{r}
\end{array}\right), \quad \dot{\mathbf{y}}:=\left(\begin{array}{c}
\dot{y}_{1} \\
\vdots \\
\dot{y}_{r}
\end{array}\right),
$$

where $\dot{x}_{i}, \dot{y}_{i}$ denote the derivatives of $x_{i}, y_{i}$ with respect to $t_{i}$. Moreover, we write $\mathfrak{m}$ for the maximal ideal of $R, \overline{\mathfrak{m}}$ for the Jacobson radical of $\bar{R}$, and

$$
\overline{\mathfrak{m}}^{\mathbf{m}}:=\bigoplus_{i=1}^{r} t_{i}^{m_{i}} K\left[\left[t_{i}\right]\right] \subset \bigoplus_{i=1}^{r} K\left[\left[t_{i}\right]\right]=\bar{R} .
$$

We define the following $R$-modules:

$$
\begin{aligned}
& M_{\bar{R} \leftarrow P}^{0}:=(\bar{R} \oplus \bar{R}) /(\bar{R} \cdot(\dot{\mathbf{x}}, \dot{\mathbf{y}})+(R \oplus R)), \\
& M_{\bar{R} \leftarrow P}^{\mathbf{m}}:=\left(\overline{\mathfrak{m}}^{\mathbf{m}} \oplus \overline{\mathfrak{m}}^{\mathbf{m}}\right) /(\overline{\mathfrak{m}} \cdot(\dot{\mathbf{x}}, \dot{\mathbf{y}})+(\mathfrak{m} \oplus \mathfrak{m})), \quad 1 \leqslant m_{i} \leqslant \operatorname{ord} \varphi_{i} .
\end{aligned}
$$

Proposition 2.8. With the above notation, we have the following:

(1) There is an isomorphism $T_{\bar{R} \leftarrow P}^{1, \mathbf{m}} \cong M_{\bar{R} \leftarrow P}^{\mathbf{m}}$.

(2) Let $\varphi_{A}: A[[x, y]] \rightarrow \bigoplus_{i=1}^{r} A\left[\left[t_{i}\right]\right]$ define an $\mathbf{m}$-multiple deformation of the parametrization (with trivial sections) over $A=K[[\mathbf{T}]]=K\left[\left[T_{1}, \ldots, T_{k}\right]\right]$, given by power series $X_{i}\left(\mathbf{T}, t_{i}\right)$, $Y_{i}\left(\mathbf{T}, t_{i}\right), i=1, \ldots, r$. Then this deformation is a versal (respectively, semiuniversal) $\mathbf{m}$-multiple deformation if and only if the column vectors

$$
\left(\frac{\partial X_{i}}{\partial T_{j}}\left(\mathbf{0}, t_{i}\right), \frac{\partial Y_{i}}{\partial T_{j}}\left(\mathbf{0}, t_{i}\right)\right)_{i=1, \ldots, r} \in \overline{\mathfrak{m}}^{\mathbf{m}} \oplus \overline{\mathfrak{m}}^{\mathbf{m}}, \quad j=1, \ldots, k,
$$

represent a system of generators (respectively, a basis) for the vector space $M_{\bar{R}} \leftarrow P$.

(3) Let $\mathbf{a}^{(j)}, \mathbf{b}^{(j)} \in \overline{\mathfrak{m}}^{\mathbf{m}}=\bigoplus_{i=1}^{r} t_{i}^{m_{i}} K\left[\left[t_{i}\right]\right]$ be such that

$$
\left(\mathbf{a}^{(j)}, \mathbf{b}^{(j)}\right)=\left(\left(\begin{array}{c}
a_{1}^{(j)} \\
\vdots \\
a_{r}^{(j)}
\end{array}\right),\left(\begin{array}{c}
b_{1}^{(j)} \\
\vdots \\
b_{r}^{(j)}
\end{array}\right)\right), \quad j=1, \ldots, k,
$$

represent a basis (respectively, a system of generators) for $M_{\bar{R}} \mathbf{m}_{P}$. Then the deformation (with trivial sections) of the parametrization over $K[[\mathbf{T}]]=K\left[\left[T_{1}, \ldots, T_{k}\right]\right]$ defined by

$$
\begin{aligned}
& X_{i}\left(\mathbf{T}, t_{i}\right)=x_{i}\left(t_{i}\right)+\sum_{j=1}^{k} a_{i}^{(j)}\left(t_{i}\right) \cdot T_{j}, \\
& Y_{i}\left(\mathbf{T}, t_{i}\right)=y_{i}\left(t_{i}\right)+\sum_{j=1}^{k} b_{i}^{(j)}\left(t_{i}\right) \cdot T_{j},
\end{aligned}
$$

$i=1, \ldots, r$, is a semiuniversal (respectively, versal) $\mathbf{m}$-multiple deformation of the parametrization.

In particular, m-multiple deformations of the parametrization are unobstructed, and they have a smooth semiuniversal base space of dimension $\operatorname{dim}_{K}\left(M_{\bar{R} \leftarrow P}^{\mathrm{m}}\right)$.

We omit the proof, because it is similar to (but simpler than) the proof of the analogous statement for equisingular deformations (Theorem 3.1).

At the end of this section, we consider the problem of the uniqueness of the sections. This depends on the characteristic of $K$. 


\section{A. Campillo, G.-M. Greuel and C. Lossen}

LEMma 2.9. If the characteristic of $K$ is good, then the forgetful natural transformation $\mathcal{D} e f \frac{\mathrm{em}}{\bar{R}} P \rightarrow$

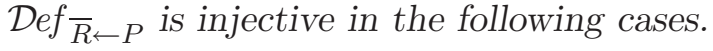

Case 1. All branches of $R$ are singular.

Case 2. The branches of $R$ have pairwise different tangent directions.

In other words, in these cases the equimultiple sections $\bar{\sigma}$ and $\sigma$ are uniquely determined (if they exist).

Proof. By [Wah74b, Proposition 1.1.4], it suffices to show the injectivity on the tangent level, that is, for $A=K[\varepsilon]$. Assume first that $R$ is irreducible, mult $(R)=m \geqslant 2$. Assume further that $X=$ $x(t)+\varepsilon x_{\varepsilon}(t), Y=y(t)+\varepsilon y_{\varepsilon}(t), x_{\varepsilon}, y_{\varepsilon} \in K[[t]]$, defines an em-deformation along the trivial sections, that is, $\min \left\{\operatorname{ord}_{t}\left(x_{\varepsilon}\right), \operatorname{ord}_{t}\left(x_{\varepsilon}\right)\right\} \geqslant m$. If $X, Y$ defines also an em-deformation with the sections $\bar{\sigma}, \sigma$ given by $I_{\bar{\sigma}}=\left\langle t+\varepsilon \gamma_{0}\right\rangle, I_{\sigma}=\left\langle x+\varepsilon \alpha_{0}, y+\varepsilon \beta_{0}\right\rangle, \alpha_{0}, \beta_{0}, \gamma_{0} \in K$, then the equimultiplicity condition $\varphi_{A}\left(I_{\sigma}\right) \subset I_{\bar{\sigma}}^{m}$ is equivalent to

$$
\begin{aligned}
x(t)+\varepsilon x_{\varepsilon}(t)+\varepsilon \alpha_{0} & =\left(h(t)+\varepsilon h_{\varepsilon}(t)\right) \cdot\left(t^{m}+m \varepsilon \gamma_{0} t^{m-1}\right) \\
y(t)+\varepsilon y_{\varepsilon}(t)+\varepsilon \beta_{0} & =\left(k(t)+\varepsilon k_{\varepsilon}(t)\right) \cdot\left(t^{m}+m \varepsilon \gamma_{0} t^{m-1}\right),
\end{aligned}
$$

for some $h, h_{\varepsilon}, k, k_{\varepsilon} \in K[[t]]$. Comparing coefficients, this implies $\alpha_{0}=\beta_{0}=0$ and, if the characteristic is either zero or if it does not divide $m$, then $\gamma_{0}=0$, too.

The injectivity in Case 1 follows immediately from these considerations, since an em-deformation of the parametrization of $R$ induces by definition em-deformations of the parametrizations of the branches $R_{i}$.

In Case 2 we may assume that at least one of the branches, say $R_{1}$ is non-singular (otherwise, Case 1 applies). Then, for each fixed $j \in\{2, \ldots, r\}$, we may choose coordinates $x, y$ such that $R_{1}$ is parametrized by $\left(x_{1}, y_{1}\right)=\left(t_{1}, 0\right)$ and $R_{j}$ has the tangent direction $x=0$. Let $X, Y$ define an em-deformation with trivial sections, and also an em-deformation with the sections $\bar{\sigma}, \sigma$ given by $I_{\bar{\sigma}_{i}}=\left\langle t+\varepsilon \gamma_{i}\right\rangle, I_{\sigma}=\left\langle x+\varepsilon \alpha_{0}, y+\varepsilon \beta_{0}\right\rangle, \alpha_{0}, \beta_{0}, \gamma_{i} \in K$. Then, similar to the above,

$$
\begin{aligned}
t+\varepsilon t a_{1, \varepsilon}(t)+\varepsilon \alpha_{0} & =\left(h_{1}(t)+\varepsilon h_{1, \varepsilon}(t)\right) \cdot\left(t+\varepsilon \gamma_{1}\right), \\
0+\varepsilon t b_{1, \varepsilon}(t)+\varepsilon \beta_{0} & =\left(k_{1}(t)+\varepsilon k_{1, \varepsilon}(t)\right) \cdot\left(t+\varepsilon \gamma_{0}\right),
\end{aligned}
$$

which implies $\alpha_{0}=\gamma_{1}$ and $\beta_{0}=0$. If the branch $R_{j}$ is singular, Case 1 shows that $\gamma_{j}=\alpha_{0}=0$, hence the uniqueness. Thus, we can assume that $R_{j}$ is smooth and parametrized by $\left(x_{j}, y_{j}\right)=\left(0, t_{j}\right)$. Then the same reasoning as above gives $\beta_{0}=\gamma_{j}$ and $\alpha_{0}=0$, thus the uniqueness of the sections.

However, there are examples of em-deformations such that both sections $\bar{\sigma}$ and $\sigma$ are not unique.

Example 2.10. (1) Let $\operatorname{char}(K)=p>0$, and consider the irreducible singularity $R=K[[x, y]] /$ $\left\langle y^{p}-x^{2 p+1}\right\rangle$. Then $(X, Y)=\left(t^{p}, t^{p}(t-\alpha)^{p+1}\right), \alpha \in \mathfrak{m}_{A}$, defines an em-deformation of the parametrization with trivial sections over $A$. However, it also defines an em-deformation with the sections $\bar{\sigma}, \sigma$ given by $I_{\bar{\sigma}}=\langle t-\alpha\rangle, I_{\sigma}=\left\langle x-\alpha^{p}, y\right\rangle$.

(2) Let $R=K[[x, y]] /\left\langle x^{4}-y^{2}\right\rangle$, which decomposes into two smooth branches with the same tangent direction. Then, for each $\alpha \in \mathfrak{m}_{A},\left(X_{1}, Y_{1}\right)=\left(t_{1},-t_{1}^{2}+\alpha t_{1}\right),\left(X_{2}, Y_{2}\right)=\left(t_{2}, t_{2}^{2}-\alpha t_{2}\right)$, defines an em-deformation with trivial sections. In addition, it defines an em-deformation with sections $\bar{\sigma}, \sigma$ given by $I_{\bar{\sigma}_{1}}=\left\langle t_{1}-\alpha\right\rangle, I_{\bar{\sigma}_{2}}=\left\langle t_{2}-\alpha\right\rangle, I_{\sigma}=\langle x-\alpha, y\rangle$.

Note that none of these examples defines an equisingular deformation. Indeed, after formally blowing up the sections, we do not get an equimultiple deformation of the strict transform. In the second example, this is caused by the fact that the sections do not satisfy the compatibility condition of Definition 2.6(iii). 
Proposition 2.11. If the characteristic of $K$ is good, and if $R$ is singular, then the forgetful functor $\operatorname{Def} \overline{\mathrm{es}}_{\leftarrow P} \rightarrow \mathcal{D} e f_{\bar{R} \leftarrow P}$ is injective. That is, the equisingular sections $\bar{\sigma}$ and $\sigma$ are unique.

Proof. If either all branches of $R$ are singular, or if they have pairwise different tangent directions, the uniqueness of the sections is implied by Lemma 2.9. Moreover, the proof of Lemma 2.9 shows that the section $\sigma$ is uniquely determined if $R$ either has a singular branch $R_{j}$ or two smooth branches $R_{i}, R_{j}, i \neq j$, intersecting transversally. Moreover, the proof shows that if the branch $R_{j}$ is either singular, or smooth and transversal to some other branch, the section $\bar{\sigma}_{j}$ is uniquely determined. It remains to consider smooth branches that are tangential to all of the other branches. After finitely many formal blow-ups the strict transform of such a branch $R_{i}$ becomes transversal to one of the other branches. Thus, the section $\bar{\sigma}_{i}$ is uniquely defined, and hence also $\sigma$ (see the proof of Lemma 2.9).

There are examples of equisingular deformations in bad characteristic $p>0$ where $\bar{\sigma}$ is not unique. For instance, the trivial deformation over $K[\varepsilon]$ of the parametrization $\left(t^{p+1}, t^{p}\right)$ of $R=$ $K[[x, y]] /\left\langle x^{p}-y^{p+1}\right\rangle$ is equisingular along the trivial sections and along the section defined by $I_{\bar{\sigma}}=\langle t+\varepsilon\rangle$. However, the section $\sigma$ is always unique as demonstrated by the following.

Proposition 2.12. Let $A \in \mathscr{A}_{K}$, and let $\varphi_{A}: A[[x, y]] \rightarrow \bigoplus_{i=1}^{r} A\left[\left[t_{i}\right]\right]$ define a deformation of $P \rightarrow \bar{R}$. If $R$ is singular, then for at most one $\sigma: A[[x, y]] \rightarrow A$ there is a lifting $\bar{\sigma}$ such that $\left(\varphi_{A}, \bar{\sigma}, \sigma\right)$ is equisingular.

Proof. From the considerations in the proofs of Lemma 2.9 and Proposition 2.11, it is clear that it suffices to consider the case of an irreducible singularity $R$ such that the multiplicity of $R$ and of all of its singular strict transforms are divisible by $p=\operatorname{char}(K)>0$. Indeed, it suffices to consider the last singular strict transform of $R$. It has a parametrization $(x, y)=\left(t^{k p}, t^{k p+1}\right)+$ higher terms, $k \geqslant 1$.

Let $X, Y$ define an em-deformation of $(x, y)$ with trivial sections over $A \in \mathscr{A}_{K}$. Then, up to terms of $t$-order $k p+1$, respectively $k p+2$, we have $X(t)=(1+a) t^{k p}, Y(t)=b_{1} t^{k p}+\left(1+b_{2}\right) t^{k p+1}$ for some $a, b_{1}, b_{2} \in \mathfrak{m}_{A}$. If $X, Y$ is also equimultiple along the sections $\bar{\sigma}, \sigma$ defined by $I_{\bar{\sigma}}=\langle t+\gamma\rangle$, $I_{\sigma}=\langle x+\alpha, y+\beta\rangle, \alpha, \beta, \gamma \in \mathfrak{m}_{A}$, then we get (again up to terms of $t$-order $k p+1$, respectively $k p+2)$

$$
\begin{aligned}
(1+a) t^{k p}+\alpha & =\left(c_{0}+c_{1} t+\cdots+c_{k p} t^{k p}\right) \cdot\left(t^{p}+\gamma^{p}\right)^{k}, \\
b_{1} t^{k p}+\left(1+b_{2}\right) t^{k p+1}+\beta & =\left(d_{0}+d_{1} t+\cdots+d_{k p+1} t^{k p+1}\right) \cdot\left(t^{p}+\gamma^{p}\right)^{k},
\end{aligned}
$$

for some $c_{j}, d_{j} \in A$. Comparing coefficients, we get $\alpha=c_{0} \gamma^{k p}, \beta=d_{0} \gamma^{k p}$, and the conditions $0=d_{1} \gamma^{k p}, 1+b_{2}=d_{1}+e \gamma^{p}$ for some $e \in A$.

Thus, $0=\left(1+b_{2}-e \gamma^{p}\right) \cdot \gamma^{k p}$, which implies $\gamma^{k p}=0$. Together with the above equalities, this yields $\alpha=\beta=0$ as claimed.

\section{Versal equisingular deformations of the parametrization}

In this section, we give a proof for the existence of a semiuniversal equisingular deformation of the parametrization and show that it has an algebraic representative with a smooth base. Moreover, we show that equisingular versality is an open property.

Let $T_{\bar{R} \leftarrow P}^{1, \text { es }}=\underline{\mathcal{D} e f} \frac{\text { es }}{\bar{R} \leftarrow R}(K[\varepsilon])$ denote the tangent space to the functor $\underline{\mathcal{D} e f} \frac{\mathrm{es}}{\bar{R} \leftarrow R}$. It is a subspace of $T_{\bar{R} \leftarrow P}^{1, \mathrm{sec}}=(\overline{\mathfrak{m}} \oplus \overline{\mathfrak{m}}) /(\overline{\mathfrak{m}}(\dot{\mathbf{x}}, \dot{\mathbf{y}})+(\mathfrak{m} \oplus \mathfrak{m}))$ (indeed, it is a subspace of each $T_{\bar{R} \leftarrow P}^{1, \bar{m}}$ where $\left.1 \leqslant m_{i} \leqslant \operatorname{ord} \varphi_{i}\right)$. Hence,

$$
T_{\bar{R} \leftarrow P}^{1, \mathrm{es}}=\frac{I_{\bar{R} \leftarrow P}^{\mathrm{es}}}{\overline{\mathfrak{m}} \cdot(\dot{\mathbf{x}}, \dot{\mathbf{y}})+(\mathfrak{m} \oplus \mathfrak{m})}
$$




\section{A. Campillo, G.-M. Greuel and C. Lossen}

with

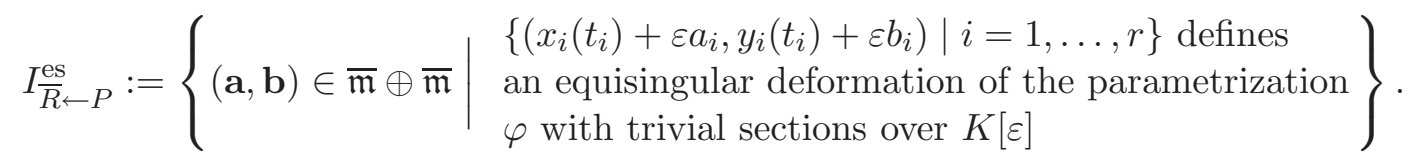

We call $I_{\bar{R} \leftarrow P}^{\mathrm{es}}$ the equisingularity module of $P \rightarrow \bar{R}$. Below, we show that it is an $R$-module (Corollary 3.5$)$.

The main theorem of this section states now that $\mathcal{D} e f \frac{\text { es }}{R \leftarrow P}$ is a 'linear' subfunctor of $\mathcal{D} e f \frac{\text { sec }}{R} \leftarrow P$. As such it is already completely determined by its tangent space.

Theorem 3.1. We have the following.

(1) Let $K[[\mathbf{T}]]=K\left[\left[T_{1}, \ldots, T_{k}\right]\right]$. Then an $r$-tuple of power series $X_{i}\left(\mathbf{T}, t_{i}\right), Y_{i}\left(\mathbf{T}, t_{i}\right) \in K\left[\left[\mathbf{T}, t_{i}\right]\right]$, $i=1, \ldots, r$, satisfying

$$
\begin{aligned}
& X_{i}\left(\mathbf{T}, t_{i}\right) \equiv x_{i}\left(t_{i}\right)+\sum_{j=1}^{k} a_{i}^{(j)}\left(t_{i}\right) \cdot T_{j} \quad \bmod \langle\mathbf{T}\rangle^{2}, \quad X_{i}(\mathbf{T}, 0)=0, \\
& Y_{i}\left(\mathbf{T}, t_{i}\right) \equiv y_{i}\left(t_{i}\right)+\sum_{j=1}^{k} b_{i}^{(j)}\left(t_{i}\right) \cdot T_{j} \quad \bmod \langle\mathbf{T}\rangle^{2}, \quad Y_{i}(\mathbf{T}, 0)=0,
\end{aligned}
$$

defines an equisingular deformation with trivial sections of the parametrization over $K[[\mathbf{T}]]$ if

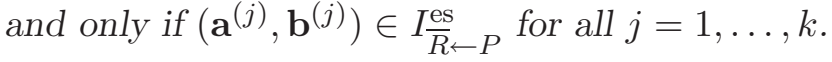

(2) Let $X_{i}\left(\mathbf{T}, t_{i}\right), Y_{i}\left(\mathbf{T}, t_{i}\right), i=1, \ldots, r$, define an equisingular deformation with trivial sections of the parametrization over $K[[\mathbf{T}]]$. Then this deformation is a versal (respectively, semiuniversal) object of $\underline{\mathcal{D} e f} \frac{\mathrm{es}}{\bar{R} \leftarrow P}$ if and only if

$$
\left(\left(\begin{array}{c}
\frac{\partial X_{1}}{\partial T_{j}}\left(\mathbf{0}, t_{1}\right) \\
\vdots \\
\frac{\partial X_{r}}{\partial T_{j}}\left(\mathbf{0}, t_{r}\right)
\end{array}\right),\left(\begin{array}{c}
\frac{\partial Y_{1}}{\partial T_{j}}\left(\mathbf{0}, t_{1}\right) \\
\vdots \\
\frac{\partial Y_{r}}{\partial T_{j}}\left(\mathbf{0}, t_{r}\right)
\end{array}\right)\right), \quad j=1, \ldots, k
$$

represent a system of $K$-generators (respectively a $K$-basis) of $T_{\bar{R} \leftarrow P}^{1, \text { es }}$.

(3) Let $\left(\mathbf{a}^{(j)}, \mathbf{b}^{(j)}\right) \in I_{\bar{R} \leftarrow P}^{\mathrm{es}}, j=1, \ldots, k$, represent a basis (respectively, a system of generators) of $T_{\bar{R} \leftarrow P}^{1, \text { es }}$. Then

$$
\begin{aligned}
& X_{i}\left(\mathbf{T}, t_{i}\right)=x_{i}\left(t_{i}\right)+\sum_{j=1}^{k} a_{i}^{(j)}\left(t_{i}\right) \cdot T_{j}, \\
& Y_{i}\left(\mathbf{T}, t_{i}\right)=y_{i}\left(t_{i}\right)+\sum_{j=1}^{k} b_{i}^{(j)}\left(t_{i}\right) \cdot T_{j},
\end{aligned}
$$

$i=1, \ldots, r$, define a semiuniversal (respectively, versal) equisingular deformation with trivial sections of the parametrization over $K[[\mathbf{T}]]$. In particular, equisingular deformations of the parametrization are unobstructed, and the semiuniversal deformation has a smooth base of dimension $\operatorname{dim}_{K} T_{\bar{R} \leftarrow P}^{1, \text { es }}$.

The proof of this theorem needs some preparation. It is based on considering small extensions in $\mathscr{A}_{K}$, that is, surjective morphisms $\widetilde{A} \rightarrow A$ of Noetherian complete local $K$-algebras with a onedimensional kernel. The generator of this kernel will be usually denoted by $\varepsilon$. Then, as $K$-vector spaces, $\widetilde{A}=A \oplus \varepsilon K$ and $\varepsilon \mathfrak{m}_{A}=0$. 


\section{EQUISINGULAR DEFORMATIONS IN ARBITRARY CHARACTERISTIC}

In Proposition 3.2 below, we show that an em-deformation of the parametrization $P \rightarrow \bar{R}$ together with a factorization through the deformation of an infinitely near point $P^{\prime}$ can be lifted to a small extension $\widetilde{A}$ of $A$. Moreover, after trivialization of the section, the lifting to the infinitely near point is determined by the lifting of the deformation of $P \rightarrow \bar{R}$ to $\widetilde{A}$.

We fix some notation: let $\widetilde{A} \rightarrow A$ be a small extension in $\mathscr{A}_{K}$, and let $\varepsilon$ denote a generator for its kernel. Let

$$
\varphi_{A}=\left(\varphi_{A, 1}, \ldots, \varphi_{A, r}\right): P_{A}=A[[x, y]] \rightarrow \bigoplus_{i=1}^{r} A\left[\left[t_{i}\right]\right]=\bar{R}_{A},
$$

$\varphi_{A, i}(x)=X_{i}\left(t_{i}\right), \varphi_{A, i}(y)=Y_{i}\left(t_{i}\right)$, define a deformation with trivial sections of the parametrization

$$
\varphi=\left(\varphi_{1}, \ldots, \varphi_{r}\right): P=K[[x, y]] \rightarrow \bigoplus_{i=1}^{r} K\left[\left[t_{i}\right]\right]=\bar{R},
$$

$\varphi_{i}(x)=x_{i}, \varphi_{i}(x)=y_{i} \in K\left[\left[t_{i}\right]\right]$. Moreover, let $P^{\prime}=K[[u, v]]$ be an infinitely near point on $R$ in the first infinitesimal neighbourhood of $P$ such that $\varphi_{i}, i \in \Lambda_{P^{\prime}}$, factors as $\varphi_{i}=\varphi_{i}^{\prime} \circ \pi^{\prime}$, where $\pi^{\prime}: K[[x, y]] \rightarrow K[[u, v]]$ is a formal blow-up of the maximal ideal in $P$. Finally, let $\pi_{A}^{\prime}: A[[x, y]] \rightarrow$ $A[[u, v]]$ be a formal blow-up of the trivial section in $P_{A}$ extending $\pi^{\prime}$. After a linear change of variables, we may assume that $\operatorname{ord}_{t_{i}} x_{i} \leqslant \operatorname{ord}_{t_{i}} y_{i}$ for all $i$, and $\pi_{A}^{\prime}(x)=u, \pi_{A}^{\prime}(y)=u(v+\beta), \beta \in A$ (see Remark 2.7(5)).

Proposition 3.2. Assume that $\left(\varphi_{A}, \bar{\sigma}, \sigma\right)$ is equimultiple and that the components $\varphi_{A, i}, i \in \Lambda_{P^{\prime}}$, of $\varphi_{A}$ factor as $\varphi_{A, i}=\varphi_{A, i}^{\prime} \circ \pi_{A}^{\prime}$ such that

$$
\varphi_{A}^{\prime}=\left(\varphi_{A, i}^{\prime}\right)_{i \in \Lambda_{P^{\prime}}}: A[[u, v]] \rightarrow \bigoplus_{i \in \Lambda_{P^{\prime}}} A\left[\left[t_{i}\right]\right]
$$

defines a deformation of $P^{\prime}=K[[u, v]] \rightarrow \bigoplus_{i \in \Lambda_{P^{\prime}}} K\left[\left[t_{i}\right]\right]=: \bar{R}^{\prime}$ with trivial sections over $A$. Then there exists an extension of $\varphi_{A}^{\prime}$,

$$
\varphi_{\widetilde{A}}^{\prime}=\left(\varphi_{\widetilde{A}, i}^{\prime}\right)_{i \in \Lambda_{P^{\prime}}}: \widetilde{A}[[u, v]] \rightarrow \bigoplus_{i \in \Lambda_{P^{\prime}}} \widetilde{A}\left[\left[t_{i}\right]\right],
$$

and a formal blow-up $\pi_{\widetilde{A}}^{\prime}: \widetilde{A}[[x, y]] \rightarrow \widetilde{A}[[u, v]]$ of the trivial section $\sigma^{\prime}$ in $\widetilde{A}[[x, y]]$ extending $\pi_{A}^{\prime}$, such that the following hold:

(i) $\varphi_{\widetilde{A}}^{\prime}$ defines a deformation of $P^{\prime} \rightarrow \bar{R}^{\prime}$ with trivial sections over $\widetilde{A}$;

(ii) $\varphi_{\widetilde{A}}^{\prime} \circ \pi_{\widetilde{A}}^{\prime}$ defines an em-deformation of $P \rightarrow \bar{R}^{\prime}$ with trivial sections.

Moreover, $\varphi_{\widetilde{A}}^{\prime}$ and $\pi_{\widetilde{A}}^{\prime}$ are uniquely determined by $\varphi_{A}^{\prime}$, $\pi_{A}^{\prime}$, and $\varphi_{\widetilde{A}}^{\prime} \circ \pi_{\widetilde{A}}^{\prime}$.

In other words, the proposition states that the following diagram of solid arrows (and trivial sections) can be completed by the dotted arrows

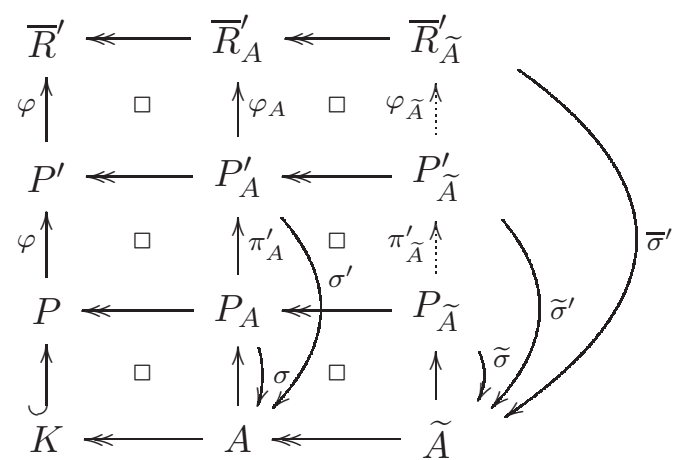

and that the dotted arrows are uniquely determined by their composition. 


\section{A. Campillo, G.-M. Greuel and C. Lossen}

Proof. Step 1: uniqueness. Assume that we have extensions $\varphi_{\widetilde{A}}^{\prime}, \pi_{\widetilde{A}}^{\prime}$ of $\varphi_{A}^{\prime}, \pi_{A}^{\prime}$ as in the proposition. Setting

$$
U_{i}:=\varphi_{A, i}^{\prime}(u), \quad V_{i}:=\varphi_{A, i}^{\prime}(v), \quad \widetilde{U}_{i}:=\varphi_{\widetilde{A}, i}^{\prime}(u), \quad \widetilde{V}_{i}:=\varphi_{\widetilde{A}, i}^{\prime}(v),
$$

we have $\widetilde{U}_{i}=U_{i}+\varepsilon a_{i}^{\prime}, \widetilde{V}_{i}=V_{i}+\varepsilon b_{i}^{\prime}$ for $a_{i}^{\prime}, b_{i}^{\prime} \in t_{i} K\left[\left[t_{i}\right]\right]$, and $\pi_{\widetilde{A}}^{\prime}(x)=u+\varepsilon \alpha_{\varepsilon}, \pi_{\widetilde{A}}^{\prime}(y)=\left(u+\varepsilon \alpha_{\varepsilon}\right)(v+$ $\left.\beta+\varepsilon \beta_{\varepsilon}\right)$ where $\alpha_{\varepsilon}, \beta_{\varepsilon} \in K$, with $\alpha_{\varepsilon}=\beta_{\varepsilon}=0$ if $\widetilde{\sigma}$ is trivial. Here $\pi_{A}^{\prime}(x)=u, \pi_{A}^{\prime}(y)=u(v+\beta), \beta \in A$ by Remark 2.7(2). By assumption, $\varphi_{A, i}$ factors as $\varphi_{A, i}=\varphi_{A, i}^{\prime} \circ \pi_{A}^{\prime}$. Hence, $X_{i}=\varphi_{A, i}(x)=U_{i}$, $Y_{i}=\varphi_{A, i}(y)=U_{i} \cdot\left(V_{i}+\beta\right)$.

Setting $\widetilde{X}_{i}:=\varphi_{\widetilde{A}, i}^{\prime} \circ \pi_{\widetilde{A}}^{\prime}(x)$, and $\widetilde{Y}_{i}:=\varphi_{\widetilde{A}, i}^{\prime} \circ \pi_{\widetilde{A}}^{\prime}(y)$, we get from condition (ii) that

$$
\widetilde{X}_{i}=X_{i}+\varepsilon a_{i}, \quad \tilde{Y}_{i}=Y_{i}+\varepsilon b_{i}
$$

for some $a_{i}, b_{i} \in t_{i} K\left[\left[t_{i}\right]\right]$ and

$$
\widetilde{X}_{i}=U_{i}+\varepsilon a_{i}^{\prime}+\varepsilon \alpha_{\varepsilon}, \quad \widetilde{Y}_{i}=\left(U_{i}+\varepsilon a_{i}^{\prime}+\varepsilon \alpha_{\varepsilon}\right) \cdot\left(V_{i}+\varepsilon b_{i}^{\prime}+\beta+\varepsilon \beta_{\varepsilon}\right) .
$$

Comparing coefficients, this implies

$$
a_{i}=a_{i}^{\prime}+\alpha_{\varepsilon}, \quad b_{i}=\left(a_{i}^{\prime}+\alpha_{\varepsilon}\right)\left(v_{i}+\beta_{0}\right)+\left(b_{i}^{\prime}+\beta_{\varepsilon}\right) u_{i},
$$

where $u_{i}=x_{i}, v_{i} \in t_{i} K\left[\left[t_{i}\right]\right]$ (respectively, $\beta_{0} \in K$ ) are the residues of $U_{i}, V_{i}$ (respectively, $\beta \bmod$ $\left.\mathfrak{m}_{A}\right)$. Since $a_{i}(0)=a_{i}^{\prime}(0)=0$ this implies $\alpha_{\varepsilon}=0$, hence

$$
a_{i}^{\prime}=a_{i}, \quad b_{i}^{\prime}=\frac{b_{i}-a_{i}\left(v_{i}+\beta_{0}\right)}{x_{i}}-\beta_{\varepsilon} .
$$

Since $b_{i}^{\prime}(0)=0$, this implies that

$$
\beta_{\varepsilon}=\left(\frac{b_{i}-a_{i}\left(v_{i}+\beta_{0}\right)}{x_{i}}\right)(0)=\frac{b_{i}}{x_{i}}(0)+\frac{a_{i} \beta_{0}}{x_{i}}(0) .
$$

In particular, the expression on the right-hand side does not depend on the choice of $i \in \Lambda_{P^{\prime}}$. Moreover, (3.1) and (3.2) show that $\widetilde{U}_{i}, \widetilde{V}_{i}, \widetilde{X}_{i}, \widetilde{Y}_{i}$ determine $a_{i}^{\prime}, b_{i}^{\prime}, \alpha_{\varepsilon}, \beta_{\varepsilon}$.

Step 2: existence. Using the above notation, we choose for each $i \in \Lambda_{P^{\prime}}$ power series $a_{i}, b_{i} \in K\left[\left[t_{i}\right]\right]$ satisfying $\operatorname{ord}_{t_{i}} a_{i}, \operatorname{ord}_{t_{i}} b_{i} \geqslant \operatorname{ord} \varphi_{i}=\operatorname{ord}_{t_{i}} x_{i}$ and the compatibility condition

$$
\left(\frac{b_{i}-a_{i} \beta_{0}}{x_{i}}\right)(0)=\left(\frac{b_{j}-a_{j} \beta_{0}}{x_{j}}\right)(0) \quad \text { for all } i, j \in \Lambda_{P^{\prime}} .
$$

Since $v_{i}(0)=0$, this allows us to define the needed extensions according to (3.2) and (3.1).

Remark 3.3. The uniqueness statement of Proposition 3.2 can be reformulated as follows: let $\widetilde{A} \rightarrow A$ be a small extension in $\mathscr{A}_{K}$, and let $\widetilde{\xi}=\left(\varphi_{\widetilde{A}}, \bar{\sigma}, \sigma\right) \in \mathcal{D} e \mathrm{f}_{\bar{R} \leftarrow P}(\widetilde{A})$ be a lifting of $\xi \in \mathcal{D} e f_{\bar{R} \leftarrow P}^{\mathrm{em}}(A)$. Further, let $\pi_{\widetilde{A}}^{\prime}: P_{\widetilde{A}} \rightarrow P_{\widetilde{A}}^{\prime}$ be a formal blow-up of the section $\sigma$. Then there is a unique morphism $\varphi_{\widetilde{A}}^{\prime}$ as in the above diagram and at most one section $\sigma^{\prime}: P_{\widetilde{A}}^{\prime} \rightarrow A$ such that $\left(\varphi_{\widetilde{A}}^{\prime}, \bar{\sigma}^{\prime}, \sigma^{\prime}\right) \in \mathcal{D}$ ef $\bar{R}_{\bar{R}^{\prime}}^{\mathrm{sec}}(\widetilde{A})$. Indeed, the diagram shows that $\sigma^{\prime}$ exists if and only if the composition $\bar{\sigma}_{i}^{\prime} \circ \varphi_{\widetilde{A}}^{\prime}$ is independent of the choice of $i \in \Lambda_{P^{\prime}}$.

As a corollary of the proof of Proposition 3.2 (applied to $A=K, \widetilde{A}=K[\varepsilon]$ ), we obtain the following lemma which allows us to argue by induction.

LEMMA 3.4.

(1) Given $a_{i}^{\prime}, b_{i}^{\prime} \in t_{i} K\left[\left[t_{i}\right]\right], i \in \Lambda_{P^{\prime}}$, let

$$
a_{i}=a_{i}^{\prime}, \quad b_{i}=a_{i}^{\prime}\left(\varphi_{i}^{\prime}(y)+\beta_{0}\right)+\left(b_{i}^{\prime}+\beta_{\varepsilon}\right) x_{i},
$$

where $\beta_{0}=\left(\beta \bmod \mathfrak{m}_{A}\right), \beta_{\varepsilon} \in K$. Then the $a_{i}, b_{i}, i \in \Lambda_{P^{\prime}}$, define an element of $I_{\bar{R}^{\prime} \leftarrow P}^{\text {es }}$ if and

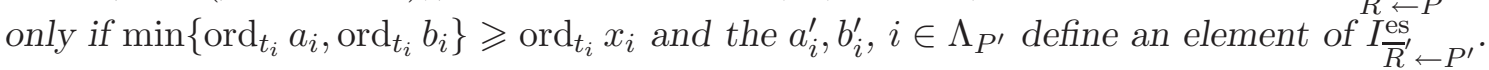


(2) Let $a_{i}, b_{i} \in t_{i} K\left[\left[t_{i}\right]\right], i \in \Lambda_{P^{\prime}}$ define an element of $I_{\bar{R}^{\prime} \leftarrow P}^{\mathrm{es}}$. Then there exists a unique $\beta_{\varepsilon} \in K$ such that setting

$$
a_{i}^{\prime}=a_{i}, \quad b_{i}^{\prime}=\frac{b_{i}-a_{i}\left(\varphi_{i}^{\prime}(y)+\beta_{0}\right)}{x_{i}}-\beta_{\varepsilon} .
$$

defines an element of $I_{\bar{R}^{\prime} \leftarrow P}^{\mathrm{es}}$ satisfying (3.3).

Note that $\bar{R}=\bigoplus_{P^{\prime}} \bar{R}^{\prime}$, hence $I_{\bar{R} \leftarrow P}^{\mathrm{es}}=\bigoplus_{P^{\prime}} I_{\bar{R}^{\prime} \leftarrow P}^{\mathrm{es}}$ where the sum on the right-hand side is taken over all points $P^{\prime}$ on $R$ in the first neighbourhood of $P$.

COROLlary 3.5. The equisingularity module $I_{\bar{R} \leftarrow P}^{\mathrm{es}}$ is an $R$-submodule of $\overline{\mathfrak{m}} \oplus \overline{\mathfrak{m}}$.

Proof. We argue by induction on the number of blow-ups needed to resolve the singularity $R$. If $R$ is regular, $I_{\bar{R} \leftarrow P}^{\mathrm{es}}=\overline{\mathfrak{m}} \oplus \overline{\mathfrak{m}}$. If $R$ is singular, we may blow up the maximal ideal in $P$ and consider the strict transform $R_{i}^{\prime}$ at each infinitely near point $P^{\prime}$ in the first neighbourhood of $P$ on $R$. By the induction hypothesis, for each such $P^{\prime}, I_{\bar{R}^{\prime} \leftarrow P^{\prime}}^{\text {es }}$ is an $R$-submodule of $\overline{\mathfrak{m}}^{\prime} \oplus \overline{\mathfrak{m}}^{\prime}$, where $\overline{\mathfrak{m}}^{\prime}=\bigoplus_{i \in \Lambda_{P^{\prime}}} \mathfrak{m}_{\bar{R}_{i}}$. Then Lemma 3.4 shows that the same holds for $I_{\bar{R}^{\prime} \leftarrow P}^{\text {es }}$. Indeed, the $K$-vector space structure is obvious. Let $h \in R$ and $(\mathbf{a}, \mathbf{b}) \in I_{\bar{R}^{\prime} \leftarrow P}^{\mathrm{es}}$. Then, according to (3.1) and (3.2), we have $\left(h a_{i}\right)^{\prime}=h a_{i}$ and $\left(h b_{i}\right)^{\prime}-h b_{i}^{\prime}=\left(h\left(\varphi_{i}^{\prime}(x), \varphi_{i}^{\prime}(y)\right)-h(0)\right) \cdot \beta_{\varepsilon} \in \mathfrak{m}_{R_{i}^{\prime}}$. Since $\mathfrak{m}_{R^{\prime}} \oplus \mathfrak{m}_{R^{\prime}} \subset I_{\bar{R}^{\prime} \leftarrow P^{\mathrm{es}}}, \mathfrak{m}_{R^{\prime}}=\bigoplus_{i \in \Lambda_{P^{\prime}}} \mathfrak{m}_{R_{i}^{\prime}}$, and since $h\left(b_{i}^{\prime}\right)_{i \in \Lambda_{P^{\prime}}} \in I_{\bar{R}^{\prime} \leftarrow P}^{\mathrm{es}}$ by the induction hypothesis, we have $\left(\left(h b_{i}\right)^{\prime}\right)_{i \in \Lambda_{P^{\prime}}} \in I_{\bar{R}^{\prime} \leftarrow P^{\text {es }}}^{\mathrm{es}}$. Then Lemma 3.4 implies that $\stackrel{R}{h \mathbf{b} \in P} \in I_{\bar{R}} \leftarrow P$, which proves the claim.

Lemma 3.6. Let the deformation with trivial sections over $A$ defined by $\varphi_{A}$ be equisingular, and let $(\mathbf{a}, \mathbf{b}) \in \overline{\mathfrak{m}} \oplus \overline{\mathfrak{m}}$. Then the deformation with trivial sections $\left(\varphi_{\widetilde{A}}, \bar{\sigma}, \sigma\right)$ over $\widetilde{A}$ defined by $\widetilde{X}_{i}:=X_{i}+\varepsilon a_{i}$, $\widetilde{Y}_{i}:=X_{i}+\varepsilon b_{i}, i=1, \ldots, r$, is equisingular if and only if $(\mathbf{a}, \mathbf{b}) \in I_{\bar{R} \leftarrow P}^{\mathrm{es}}$.

Proof. We argue again by induction on the number of blow-ups needed to resolve the singularity $R$. If $R$ is regular, the statement is obvious. Now, assume that $R$ is singular.

If the deformation $\left(\varphi_{\widetilde{A}}, \bar{\sigma}, \sigma\right)$ is equisingular, we find a formal blow-up $\pi_{\widetilde{A}}^{\prime}$ of the trivial section $\sigma$ in $P_{A}=A[[x, y]]$ such that, for each point $P^{\prime}=K[[u, v]]$ in the first neighbourhood of $P$ on $R$, and for each $i \in \Lambda_{P^{\prime}}$, the morphism $\varphi_{\widetilde{A}, i}$ factors as $\varphi_{\widetilde{A}, i}=\varphi_{\widetilde{A}, i}^{\prime} \circ \pi_{\widetilde{A}}^{\prime}$. Moreover, we can assume that the $\varphi_{\widetilde{A}, i}^{\prime}: A[[u, v]] \rightarrow K\left[\left[t_{i}\right]\right], i \in \Lambda_{P^{\prime}}$ define an equisingular deformation with trivial sections of the parametrization $P^{\prime} \rightarrow \bar{R}^{\prime}$. Setting, as before, $\varphi_{\widetilde{A}, i}(u)=: U_{i}+\varepsilon a_{i}^{\prime}, \varphi_{\widetilde{A}, i}(v)=: V_{i}+\varepsilon b_{i}^{\prime}$, the induction hypothesis gives that the $a_{i}^{\prime}, b_{i}^{\prime}, i \in \Lambda_{P^{\prime}}$ define an element of $I_{\bar{R}^{\prime} \leftarrow P^{\prime}}^{\mathrm{es}}$. Since they necessarily satisfy the equality (3.3), Lemma 3.4(a) implies that $(\mathbf{a}, \mathbf{b}) \in I_{\bar{R} \leftarrow P}^{\mathrm{es}}$. Note that the condition on the order of $a_{i}, b_{i}$ is satisfied, since $\widetilde{X}_{i}, \widetilde{Y}_{i}$ defines an equisingular deformation along the trivial section.

Conversely, let us assume that $(\mathbf{a}, \mathbf{b}) \in I_{\bar{R}} \leftarrow P$. Then $\left(\varphi_{\widetilde{A}}, \bar{\sigma}, \sigma\right)$ is obviously equimultiple. Moreover, choosing $a_{i}^{\prime}, b_{i}^{\prime} \in K\left[\left[t_{i}\right]\right]$ according to $(3.4), U_{i}+\varepsilon a_{i}^{\prime}, V_{i}+\varepsilon b_{i}^{\prime}$ defines a deformation with trivial sections of the parametrization $P^{\prime} \rightarrow \bar{R}^{\prime}$ which is obtained from $\varphi_{\widetilde{A}}$ via a formal blow-up (see the proof of Proposition 3.2). Lemma 3.4(b) together with the induction hypothesis give that this deformation is, indeed, equisingular.

Lemma 3.7. We have $\left(\varphi_{A}, \bar{\sigma}, \sigma\right) \in \operatorname{Def} \frac{\mathrm{sec}}{\bar{R} \leftarrow P}(A)$ is equisingular if and only if it is formally equisingular, that is, if and only if, for each $N \geqslant 1$, the induced deformation with sections over $A / \mathfrak{m}_{A}^{N}$ is equisingular.

Proof. The proof is straightforward, by induction on the number of formal blow-ups.

Proof of Theorem 3.1. Let $A=K[[\mathbf{T}]]$, and let $\left(\varphi_{A}, \bar{\sigma}, \sigma\right)$ denote the deformation with trivial sections defined by $X_{i}, Y_{i} \in t_{i} A\left[\left[t_{i}\right]\right]$. 


\section{A. Campillo, G.-M. Greuel and C. Lossen}

(1) If $\left(\varphi_{A}, \bar{\sigma}, \sigma\right)$ is equisingular, then, for each $j=1, \ldots, k$, the deformation $\left(\psi \varphi_{A}, \psi \bar{\sigma}, \psi \sigma\right)$ induced by the projection $\psi: K[[\mathbf{T}]] \rightarrow K[[\mathbf{T}]] /\left(\left\langle T_{j}^{2}\right\rangle+\left\langle T_{\ell} \mid \ell \neq j\right\rangle\right)$ is equisingular, too. Thus, Lemma 3.6 gives $\left(\mathbf{a}^{(j)}, \mathbf{b}^{(j)}\right) \in I_{\bar{R} \leftarrow P^{\text {es }}}$.

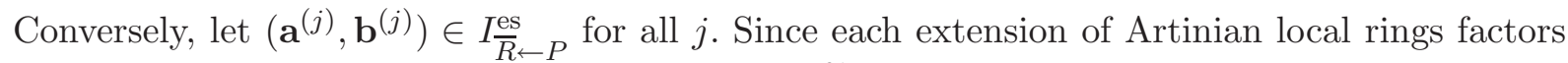
through small extensions, Lemma 3.6 (applied to $\widetilde{A}$ a small extension of $A / \mathfrak{m}^{N}, N>1$, and $\left.a_{i}=b_{i}=0\right)$ implies that $\left(\varphi_{A}, \bar{\sigma}, \sigma\right)$ is formally equisingular, hence equisingular due to Lemma 3.7.

Since part (3) is an immediate consequence of part (2), it remains to prove part $(2)$. Let $\left(\varphi_{A}, \bar{\sigma}, \sigma\right)$ be a versal (respectively semiuniversal) object of $\mathcal{D} e f \frac{\mathrm{es}}{\bar{R} \leftarrow P}$. Then, for any $(\mathbf{a}, \mathbf{b}) \in I_{\bar{R} \leftarrow P}^{\mathrm{es}}$, the equisingular deformation with trivial sections defined by $x_{i}+\varepsilon a_{i}, y_{i}+\varepsilon b_{i}, i=1, \ldots, r$, can be induced (respectively, uniquely induced) from $\left(\varphi_{A}, \bar{\sigma}, \sigma\right)$ via a morphism in $\mathscr{A}_{K}, K[[\mathbf{T}]] \rightarrow K[\varepsilon], T_{j} \mapsto \beta_{j} \varepsilon$. However, this means that $x_{i}+\varepsilon a_{i}=X_{i}\left(\beta \varepsilon, t_{i}\right), y_{i}+\varepsilon b_{i}=Y_{i}\left(\beta \varepsilon, t_{i}\right)$ for some (respectively, for a unique) $\beta=\left(\beta_{1}, \ldots, \beta_{k}\right)$. Expanding and comparing coefficients, we get

$$
\left(a_{i}, b_{i}\right)=\sum_{j=1}^{k} \beta_{j}\left(\frac{\partial X_{i}}{\partial T_{j}}\left(\mathbf{0}, t_{i}\right), \frac{\partial Y_{i}}{\partial T_{j}}\left(\mathbf{0}, t_{i}\right)\right)
$$

thus the necessity of the condition.

To show that the given deformation is versal (semiuniversal) along the trivial sections, it suffices to show that it is formally versal (semiuniversal), according to [Fle8l, (5.2) Satz]. Thus, it is sufficient to consider a small extension $\chi: \widetilde{C} \rightarrow C$ in $\mathscr{A}_{K}$ with kernel $\varepsilon K$, and equisingular deformations $\eta, \widetilde{\eta}$ over $C, \widetilde{C}$, respectively. We assume that $\eta$ is induced from $\widetilde{\eta}$ by $\underset{\sim}{\chi}$ and from $\xi$ by a morphism $\psi: A \rightarrow C$ in $\mathscr{A}_{K}$, and have to show that there exists a morphism $\widetilde{\psi}: A \rightarrow \widetilde{C}$ such that $\chi \circ \widetilde{\psi}=\psi$ and that $\widetilde{\eta}$ can be induced from $\xi$ via $\widetilde{\psi}$. To show the semiuniversality, we have to show additionally that the tangent map of $\widetilde{\psi}$ is uniquely determined.

We introduce the following notation:

- $\eta$ is given by $W_{i}\left(t_{i}\right), Z_{i}\left(t_{i}\right) \in C\left[\left[t_{i}\right]\right]$;

- $\widetilde{\eta}$ is given by $\widetilde{W}_{i}\left(t_{i}\right)=W_{i}\left(t_{i}\right)+\varepsilon w_{i}^{\varepsilon}, \widetilde{Z}_{i}\left(t_{i}\right)+\varepsilon z_{i}^{\varepsilon}$, where $w_{i}^{\varepsilon}, z_{i}^{\varepsilon} \in K\left[\left[t_{i}\right]\right]$;

- $\psi\left(T_{j}\right)=\widetilde{\psi}\left(T_{j}\right)+\varepsilon \beta_{j}^{\varepsilon}$, where $\beta_{j}^{\varepsilon} \in K$.

Then the assumption is that there are a $C$-automorphism of $C[[x, y]]$, mapping $x \mapsto H_{1}(x, y)$, $y \mapsto H_{2}(x, y), H_{1}, H_{2} \in\langle x, y\rangle C[[x, y]]$, and $C$-automorphisms of $C\left[\left[t_{i}\right]\right]$ mapping $t_{i} \mapsto s_{i} \in t_{i} C\left[\left[t_{i}\right]\right]$, $i=1, \ldots, r$, such that

$$
H_{1} \equiv x \quad \bmod \mathfrak{m}_{C}, \quad H_{2} \equiv y \quad \bmod \mathfrak{m}_{C}, \quad s_{i} \equiv t_{i} \quad \bmod \mathfrak{m}_{C}
$$

and $X_{i}\left(\psi(\mathbf{T}), t_{i}\right)=H_{1}\left(W_{i}\left(s_{i}\right), Z_{i}\left(s_{i}\right)\right), Y_{i}\left(\psi(\mathbf{T}), t_{i}\right)=H_{2}\left(W_{i}\left(s_{i}\right), Z_{i}\left(s_{i}\right)\right)$.

We show that these automorphisms can be extended to $\widetilde{C}$-automorphisms:

- $x \mapsto H_{1}+\varepsilon h_{1}^{\varepsilon}, y \mapsto H_{2}+\varepsilon h_{2}^{\varepsilon}, h_{1}^{\varepsilon}, h_{2}^{\varepsilon} \in\langle x, y\rangle K[[x, y]]$;

- $t_{i} \mapsto s_{i}+\varepsilon s_{i}^{\varepsilon}, s_{i}^{\varepsilon} \in\left\langle t_{i}\right\rangle K\left[\left[t_{i}\right]\right], i=1, \ldots, r ;$

such that

$$
\begin{aligned}
X_{i}\left(\psi(\mathbf{T})+\varepsilon \beta^{\varepsilon}, t_{i}\right) & =\left(H_{1}+\varepsilon h_{1}^{\varepsilon}\right)\left(\widetilde{W}_{i}\left(s_{i}+\varepsilon s_{i}^{\varepsilon}\right), \widetilde{Z}_{i}\left(s_{i}+\varepsilon s_{i}^{\varepsilon}\right)\right), \\
Y_{i}\left(\psi(\mathbf{T})+\varepsilon \beta^{\varepsilon}, t_{i}\right) & =\left(H_{2}+\varepsilon h_{2}^{\varepsilon}\right)\left(\widetilde{W}_{i}\left(s_{i}+\varepsilon s_{i}^{\varepsilon}\right), \widetilde{Z}_{i}\left(s_{i}+\varepsilon s_{i}^{\varepsilon}\right)\right) .
\end{aligned}
$$

Expanding the left-hand sides as power series in $\varepsilon$ (and using $\varepsilon \mathfrak{m}_{\widetilde{C}}=0$ ), we get

$$
X_{i}\left(\psi(\mathbf{T})+\varepsilon \beta^{\varepsilon}, t_{i}\right)=X_{i}\left(\psi(\mathbf{T}), t_{i}\right)+\varepsilon \sum_{j=1}^{k} \frac{\partial X_{i}}{\partial T_{j}}\left(\mathbf{0}, t_{i}\right) \cdot \beta_{j}^{\varepsilon}
$$




\section{EQUISINGULAR DEFORMATIONS IN ARBITRARY CHARACTERISTIC}

and similarly for $Y_{i}$. Note that $\widetilde{W}_{i}\left(s_{i}+\varepsilon s_{i}^{\varepsilon}\right)=W_{i}\left(s_{i}\right)+\varepsilon\left(\dot{x}_{i}\left(t_{i}\right) \cdot s_{i}^{\varepsilon}+w_{i}^{\varepsilon}\right)$, and similarly for $Z_{i}$. Expanding the right-hand side of (3.6), we get (taking into account (3.5), which implies that $\partial H_{1} / \partial x \equiv 1$ $\bmod \mathfrak{m}_{C}$ and $\left.\partial H_{1} / \partial y \equiv 0 \bmod \mathfrak{m}_{C}\right)$

$$
\begin{gathered}
\left(H_{1}+\varepsilon h_{1}^{\varepsilon}\right)\left(W_{i}\left(s_{i}\right)+\varepsilon\left(\dot{x}_{i} \cdot s_{i}^{\varepsilon}+w_{i}^{\varepsilon}\right), Z_{i}\left(s_{i}\right)+\varepsilon\left(\dot{y}_{i}\left(t_{i}\right) \cdot s_{i}^{\varepsilon}+z_{i}^{\varepsilon}\right)\right) \\
=H_{1}\left(W_{i}\left(s_{i}\right), Z_{i}\left(s_{i}\right)\right)+\varepsilon\left(h_{1}^{\varepsilon}\left(x_{i}, y_{i}\right)+\dot{x}_{i} \cdot s_{i}^{\varepsilon}+w_{i}^{\varepsilon}\right) .
\end{gathered}
$$

Comparing this with the expansion of the left-hand side of (3.6) shows that it suffices to find $\beta^{\varepsilon}, h_{1}^{\varepsilon}, h_{2}^{\varepsilon}, \mathbf{s}^{\varepsilon}$ satisfying the equality

$$
\left(w_{i}^{\varepsilon}, z_{i}^{\varepsilon}\right)=\sum_{j=1}^{k} \beta_{j}^{\varepsilon} \cdot\left(\frac{\partial X_{i}}{\partial T_{j}}\left(\mathbf{0}, t_{i}\right), \frac{\partial X_{i}}{\partial T_{j}}\left(\mathbf{0}, t_{i}\right)\right)-s_{i}^{\varepsilon} \cdot\left(\dot{x}_{i}, \dot{y}_{i}\right)-\left(h_{1}^{\varepsilon}\left(x_{i}, y_{i}\right), h_{2}^{\varepsilon}\left(x_{i}, y_{i}\right)\right) .
$$

From Lemma 3.6, we know that $\mathbf{w}^{\varepsilon}, \mathbf{z}^{\varepsilon} \in I_{\bar{R} \leftarrow P}^{\mathrm{es}}$. Hence, the assumptions imply that we find a solution $\beta^{\varepsilon}, h_{1}^{\varepsilon}, h_{2}^{\varepsilon}, \mathbf{s}^{\varepsilon}$ for the above equation (respectively, a solution with uniquely determined $\beta^{\varepsilon}$ ).

As a corollary of Theorem 3.1 and Corollary 3.5, we get the 'openness of versality' for equisingular deformations with sections of the parametrization: let $\left(S, \mathcal{O}_{S}\right)$ be an algebraic $K$-scheme, then we call a family $\mathcal{O}_{S} \rightarrow \mathcal{O}_{S}[[x, y]] \rightarrow \bigoplus_{i=1}^{r} \mathcal{O}_{S}\left[\left[t_{i}\right]\right]$ of parametrizations of reduced plane curve singularities over $S$ equisingular, if for any (closed) point $s \in S$, the induced family $\widehat{\mathcal{O}}_{S, s} \rightarrow \widehat{\mathcal{O}}_{S, s}[[x, y]] \rightarrow$ $\bigoplus_{i=1}^{r} \widehat{\mathcal{O}}_{S, s}\left[\left[t_{i}\right]\right]$ defines an equisingular deformation of the parametrization with trivial sections. We say that the family is equisingular-versal at $s$, if the induced family over the complete local ring $\widehat{\mathcal{O}}_{S, s}$ is a versal equisingular deformation of the special fibre.

Corollary 3.8. Let $\mathcal{O}_{S} \rightarrow \mathcal{O}_{S}[[x, y]] \rightarrow \bigoplus_{i} \mathcal{O}_{S}\left[\left[t_{i}\right]\right]$ be an equisingular family of parametrizations of reduced plane curve singularities. Then the set of (closed) points $s \in S$ such that the family is equisingular-versal at $s$ is open in $S$.

Proof. Let $\mathcal{J}^{\text {es }}=\mathcal{J}_{\oplus_{i}}^{\text {es }} \mathcal{O}_{S}\left[\left[t_{i}\right]\right] \leftarrow \mathcal{O}_{S}[[x, y]]$ be the subsheaf of $\bigoplus_{i}\left(t_{i} \mathcal{O}_{S}\left[\left[t_{i}\right]\right] \oplus t_{i} \mathcal{O}_{S}\left[\left[t_{i}\right]\right]\right)$ of elements $(\mathbf{a}, \mathbf{b})$ such that $X_{i}\left(t_{i}\right)+a_{i}\left(t_{i}\right), Y_{i}\left(t_{i}\right)+b_{i}\left(t_{i}\right)$ defines an equisingular family over $S$. Here, as usual, $X_{i}, Y_{i} \in \mathcal{O}_{S}\left[\left[t_{i}\right]\right]$ denote the images of $x$ and $y$ in $\mathcal{O}_{S}\left[\left[t_{i}\right]\right]$. Let $\dot{X}_{i}, \dot{Y}_{i}$ denote the partials of $X_{i}, Y_{i}$ (with respect to $t_{i}$ ). Then the quotient sheaf

$$
\mathcal{T}^{1, \mathrm{es}}=\mathcal{T}_{\bigoplus_{i} \mathcal{O}_{S}\left[\left[t_{i}\right]\right] \leftarrow \mathcal{O}_{S}[[x, y]]}^{1, \mathrm{es}}=\mathcal{J}^{\mathrm{es}} /\left(\bigoplus_{i}\left(\dot{X}_{i}, \dot{Y}_{i}\right) t_{i} \mathcal{O}_{S}\left[\left[t_{i}\right]\right]+\langle x, y\rangle \mathcal{O}_{S}[[x, y]]\right),
$$

is a coherent $\mathcal{O}_{S^{-}}$-sheaf. Moreover, we have the Kodaira-Spencer map $\Theta_{S} \rightarrow \mathcal{T}^{1 \text {,es }}$ which maps $\delta$ to the class of $\left(\delta\left(X_{i}\right), \delta\left(Y_{i}\right)\right)_{i=1}^{r}$. By Theorem 3.1, the equisingular-versal locus is the complement of the support of the cokernel of this map. Thus, the equisingular-versal locus is open in $S$.

\section{Equisingular deformations of the equation}

We now turn to deformations of the singularity itself, that is, to deformations of $R=P /\langle f\rangle$ with or without section. Analogous to Definition 1.1, they are defined by flat morphisms $A \rightarrow R_{A}$ in $\mathscr{A}_{K}$ (with section $\sigma$ ), together with a surjection $R_{A} \rightarrow R$ over $A \rightarrow K$ such that the corresponding diagram is Cartesian:

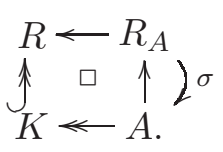

Morphisms are morphisms of diagrams. We denote the corresponding category by $\mathcal{D} f_{R}^{\mathrm{sec}}$ (respectively, $\mathcal{D} e f_{R}$ ) and the deformation functors by $\underline{\mathcal{D e f}}_{R}^{\mathrm{sec}}$ (respectively, $\underline{\operatorname{Def}}_{R}$ ). In order to distinguish 


\section{A. Campillo, G.-M. Greuel and C. Lossen}

them from deformations of the parametrization, we also refer to the objects of $\mathcal{D} e f_{R}$ (respectively, $\mathcal{D} e f_{R}^{\mathrm{sec}}$ ) as deformations of the equation (respectively, with section) since, basically, we deform $f$. From Proposition 1.3, we deduce the following commutative diagram

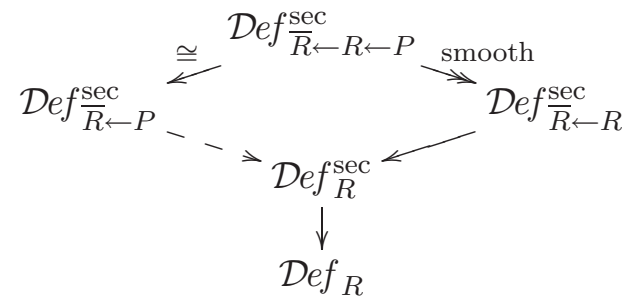

where the solid arrows are the natural forgetful functors and the dashed arrow is defined by making the diagram commutative. For the deformation functors of isomorphism classes we have

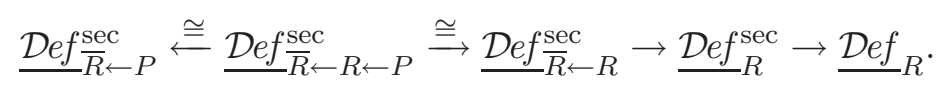

We turn now to equisingular deformations.

Definition 4.1. A deformation of $R$ is called (strongly) equisingular (or an es-deformation) if it is induced by an equisingular deformation of the parametrization of $R$. That is, we define the category $\mathcal{D} e f_{R}^{\text {es }}$ to be the full subcategory of $\mathcal{D} e f_{R}$,

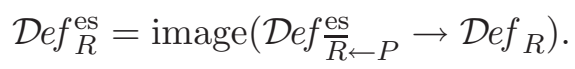

Here $\underline{\mathcal{D} e f}_{R}^{\text {es }}$ denotes the corresponding subfunctor of $\underline{\mathcal{D e f}}_{R}$. In particular, we introduce

$$
T_{R}^{1, \mathrm{es}}=\underline{\mathcal{D} e f}_{R}^{\mathrm{es}}(K[\varepsilon]) .
$$

Similarly, we define

$$
\text { Def } f_{R}^{\text {es,sec }}=\text { image }\left(\mathcal{D} e f_{\bar{R} \leftarrow P}^{\text {es }} \rightarrow \mathcal{D} e f_{R}^{\text {sec }}\right)
$$

as full subcategory of $\mathcal{D} e f_{R}^{\mathrm{sec}}$ and call objects of $\mathcal{D} e f_{R}^{\mathrm{es}, \mathrm{sec}}$ equisingular deformations of $R$ with section. The corresponding functor $\underline{D}_{R}^{\mathrm{es}, \mathrm{sec}}$ of isomorphism classes is called the equisingular deformation functor with section.

Since, by Proposition 2.12, every equisingular deformation of $P \rightarrow \bar{R}$ has a unique section $\sigma$, the forgetful functors from the image of $\mathcal{D} e \frac{\mathrm{es}}{\bar{R} \leftarrow P}$ in $\mathcal{D e f}_{R}^{\mathrm{sec}}$ to $\operatorname{Def} f_{R}$ is injective on objects, if $R$ is singular. Moreover, since the section is singular, any isomorphism in $\mathcal{D} e f_{R}$ must respect the section and, hence, lifts to an isomorphism in $\mathcal{D} e f_{R}^{\mathrm{sec}}$. That is, $\mathcal{D} e f_{R}^{\mathrm{es}}$ and $\mathcal{D} e f_{R}^{\mathrm{es}, \mathrm{sec}}$ are equivalent categories and the functors $\frac{\mathcal{D} e f}{R}{ }_{R}^{\text {es }}$ and $\underline{\mathcal{D}}_{R}^{\text {es,sec }}$ are isomorphic. In particular, the vector spaces $T_{R}^{1, \text { es }}$ and $T_{R}^{1, \text { es,sec }}$ are isomorphic (but not equal: the first is a subspace of $R / J$, the second of $\mathfrak{m} / \mathfrak{m} J$; cf. $\S 5)$.

Theorem 3.1 yields immediately the first main result of this paper.

\section{THEOREM 4.2 .}

(1) The natural transformation $\underline{\mathcal{D} e f} \overline{\mathrm{es}}_{\bar{R} \leftarrow P} \rightarrow \underline{\mathcal{D} e f}_{R}^{\mathrm{es}, \mathrm{sec}} \cong \underline{\mathcal{D} e f}_{R}^{\mathrm{es}}$ is smooth. In particular, equisingular deformations of $R$ are unobstructed and have a semiuniversal deformation with smooth base of dimension $\operatorname{dim}_{K} T_{R}^{1, \text { es }}$.

(2) $\underline{\mathcal{D} e f} \overline{\mathrm{es}}_{\bar{R} \leftarrow P} \rightarrow \underline{\mathcal{D} e f_{R}^{\text {es }}}$ is an isomorphism of functors if and only if $\operatorname{dim}_{K} T_{\bar{R} \leftarrow P}^{1, \text { es }}=\operatorname{dim}_{K} T_{R}^{1, \text { es }}$. If this holds, a semiuniversal equisingular deformation of $R$ is obtained from the semiuniversal equisingular deformation of the parametrization (as given in Theorem 3.1(3)) by elimination of the parametrizing variables.

(3) If the characteristic of $K$ is good, then $\underline{\operatorname{Def}} \frac{\mathrm{es}}{\bar{R} \leftarrow R} \cong \underline{\mathcal{D} e f}{ }_{R}^{\mathrm{es}}$, and the semiuniversal object of $\underline{D}$ ef $_{R}^{\mathrm{es}}$ has an algebraic representative. 


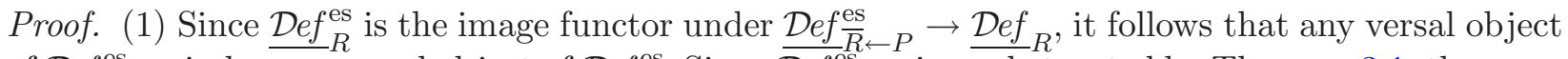

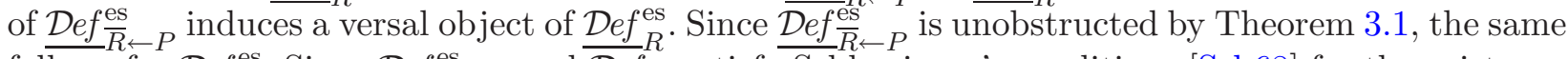
follows for $\underline{\mathcal{D e f}}_{R}^{\text {es }}$. Since $\underline{\mathcal{D} e f} \overline{\mathrm{es}}_{\leftarrow} \leftarrow P$ and $\underline{\mathcal{D e f}}_{R}$ satisfy Schlessinger's conditions [Sch68] for the existence of a formal versal deformation (the first by Theorem 3.1, for the second this is well known), the same holds for $\underline{\mathcal{D}}_{R}^{\mathrm{es}}$. Now, it follows from [Fle8l, (5.2) Satz] that $\underline{\mathcal{D} e f}_{R}^{\text {es }}$ has a semiuniversal deformation with smooth base, and that $\underline{\mathcal{D} e f} \overline{\mathrm{es}}_{R} \leftarrow P \rightarrow \underline{\mathcal{D} e f}{ }_{R}^{\mathrm{es}}$ is smooth.

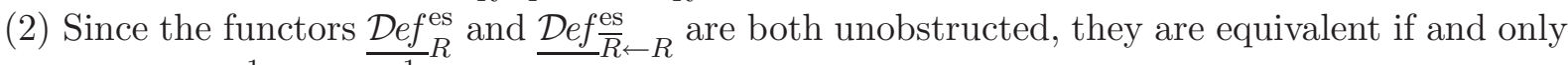
if the surjection $T_{\bar{R} \leftarrow P}^{1, \mathrm{es}} \rightarrow T_{R}^{1, \mathrm{es}}$ is an isomorphism, showing part (2).

(3) In good characteristic, Proposition 5.5 below says that $T_{R}^{1, \text { es }} \cong T_{\bar{R} \leftarrow R}^{1, \text { es }}$.

From Proposition 1.3 and its proof, we deduce that a semiuniversal object of $\frac{\mathcal{D} e f}{R}$ is obtained

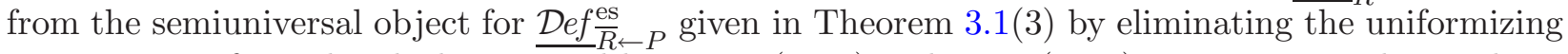
parameters $t_{i}$ from the ideal generated by $x-X_{i}\left(\mathbf{T}, t_{i}\right)$ and $y-Y_{i}\left(\mathbf{T}, t_{i}\right), i=1, \ldots, r$. The resulting power series $F_{i}(\mathbf{T}, x, y), i=1, \ldots, r$ (respectively their product $F=F_{1} \cdots F_{r}$ ), define the ideal of the total space of the semiuniversal deformations of the branches $R_{i}$ (respectively of $R$ ).

Since $R$ has an isolated singularity, it is finitely determined, hence we may assume that $x_{i}$ and $y_{i}$ are polynomials in $t_{i}$, see [Hir63, Thm. B]. Since $T_{\bar{R} \leftarrow P}^{1, \text { es }}$ is a finite-dimensional $K$-vector space, we can also choose the $a_{i}^{(j)}$ and the $b_{i}^{(j)}$ in Theorem 3.1(3) to be polynomials in $t_{i}$. Then $X_{i}, Y_{i} \in K\left[\mathbf{T}, t_{i}\right]$, and we have to eliminate $t_{i}$ from $x-X_{i}, y-Y_{i}$, that is, we have to compute a generator $F_{i}$ for the ideal $\left\langle x-X_{i}, y-Y_{i}\right\rangle \cdot K\left[\left[x, y, \mathbf{T}, t_{i}\right]\right] \cap K[[x, y, \mathbf{T}]]$.

If $p=\operatorname{char}(K)$ is good, we can compute $m_{i}$ th roots of units in $K\left[\left[t_{i}\right]\right]\left(m_{i}=\operatorname{mult}\left(R_{i}\right)\right)$, and hence we may assume that the parametrization is of the form $x_{i}\left(t_{i}\right)=t_{i}^{m_{i}}$, and $\operatorname{ord}_{t_{i}} y_{i}\left(t_{i}\right)>m_{i}$. However, then $\left\{x-X_{i}=y-Y_{i}=0\right\}$ intersects the $t_{i}$-axis only in $t_{i}=0$. Thus, we can eliminate in the polynomial ring, that is, we get $\left\langle F_{i}\right\rangle=\left\langle x-X_{i}, y-Y_{i}\right\rangle \cap K[x, y, \mathbf{T}]$ (cf. [GP02]). The product $F=F_{1} \cdots F_{r}$ defines via $K[\mathbf{T}] \rightarrow K[x, y, \mathbf{T}] /\langle F\rangle$ an algebraic representative of the semiuniversal equisingular deformation of $R$.

Remark 4.3. (1) Let $\xi$ be an object of $\mathcal{D} e f \frac{\mathrm{es}}{R \leftarrow P}(A)$. Then Proposition 1.3 shows that there exists a

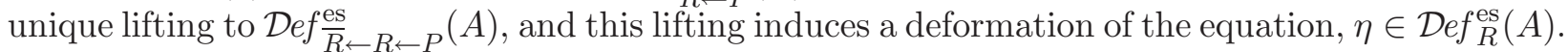
If $\xi$ is versal, then $\eta$ is versal too. If $\xi$ is semiuniversal, then, however, $\eta$ need not be semiuniversal.

More precisely, if $\xi$ is semiuniversal and if $\eta_{s} \in \mathcal{D} e f_{R}^{\text {es }}(B)$ is semiuniversal equisingular, then, by Theorem $4.2, \eta$ can be induced from $\eta_{s}$ by a map $B \rightarrow A$, where $A \cong B\left[\left[z_{1}, \ldots, z_{\ell}\right]\right]$. Here, $\ell=\operatorname{dim}_{K} \operatorname{ker}\left(T_{\bar{R} \leftarrow P}^{1, \text { es }} \rightarrow T_{R}^{1, \text { es }}\right)$, which can be computed by using the exact sequences in $\S 5$.

(2) For $K$ a field of characteristic 0, Wahl [Wah74a] introduced in a different way a functor ES of equisingular deformations of $R$ (over Artinian rings). He considered an embedded resolution (by finitely many successive blow-ups) of the singularity $R$. Then a deformation of $R$ is equisingular in the sense of Wahl, if it is an em-deformation of the equation along some section. Further he requires that, after blowing up the section, there exist sections through the infinitely near points on $R$ in the first neighbourhood of $P$ along which the blown-up family induces an equisingular deformation of the equation of the reduced total transform. Thus, the definition is by induction on the number of blow-ups needed to resolve the singularity. Any em-deformation of a node is equisingular.

Note that equimultiplicity for the parametrization (as in our definition) differs from equimultiplicity for the equation (as in Wahl's definition): an em-deformation of the parametrization induces an em-deformation of the equation, but not conversely. For instance, it can be easily seen that the em-deformation along the trivial section of the cusp $R=K[[x, y]] /\left\langle x^{2}-y^{3}\right\rangle$ given by

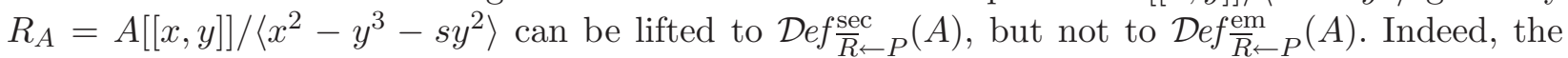
unique lifting is given by the parametrization $X(t)=t^{3}-s^{2} t, Y(t)=t^{2}-s^{2}$ which is not equimultiple along any section. 


\section{A. Campillo, G.-M. Greuel and C. Lossen}

Hence, the relation between Wahl's $\overline{\mathrm{ES}}$, the functor of isomorphism classes of ES, and our $\underline{D e f}_{R}^{\text {es }}$ is not completely obvious. Nevertheless, equisingular deformations in Wahl's sense lift to equisingular deformations of the parametrization and it can be shown that $\underline{\mathcal{D e f}}_{R}^{\text {es }}$ is isomorphic to $\overline{\mathrm{ES}}$ (cf. [GLS07]).

(3) The main result of Wahl's paper [Wah74a] is that $\overline{\mathrm{ES}}$ is unobstructed. Theorem 4.2 provides a new proof. Wahl's proof appears to be more involved than ours, as it uses deformation theory of global objects, namely of divisors supported on the exceptional divisor of the (embedded) resolution of $R$. Moreover, as Wahl shows, there is no easy description of $\overline{\mathrm{ES}}$. For example, it is not, in general, a linear subfunctor of $\underline{\mathcal{D e f}}_{R}$, the functor of deformations of $R$.

On the other hand, our functor $\underline{\mathcal{D} e f} \overline{\mathrm{es}} \leftarrow P$ is very easy to describe as a linear subfunctor of $\underline{\mathcal{D} e f} \overline{\mathrm{sec}} \leftarrow P^{\text {s }}$. Hence, even in characteristic zero, our approach to equisingular deformations of $R$ via deformations of the parametrization provides an easy understanding of the objects in $\mathcal{D} e f_{R}^{\text {es }}$.

By Theorem 4.2(2), a semiuniversal base for $\underline{\mathcal{D e f}}_{R}^{\mathrm{es}}$ is obtained from a semiuniversal base for $\underline{D} f_{\bar{R}}^{\text {es }}$ by elimination. As elimination is highly nonlinear, this 'explains' why we cannot expect $\underline{\mathcal{D} e f}_{R}^{\mathrm{es}}$ to be a linear subfunctor of $\underline{\mathcal{D} e f}{ }_{R}$.

On the other hand, Wahl introduced special equisingular deformations $\mathrm{ES}^{\prime} \subset \mathrm{ES}$ such that $\overline{\mathrm{ES}}^{\prime}$ is a linear subfunctor of $\underline{\mathcal{D} e f} R$. These special deformations lift to equisingular deformations of the parametrization for which the elimination is linear.

(4) Let $K=\mathbb{C}$. All results proved so far are valid (with the same proofs) for convergent instead of formal power series. Then we use the geometric language of deformations of reduced plane curve singularities $(C, 0)=(\{f=0\}, 0) \subset\left(\mathbb{C}^{2}, 0\right)$ over complex space germs $(T, 0)$, where $f \in \mathbb{C}\{x, y\}$. Let $(\bar{C}, \overline{0}) \rightarrow(C, 0)$ denote the normalization of $(C, 0)$, and $(\bar{C}, \overline{0}) \rightarrow(C, 0) \hookrightarrow$ $\left(\mathbb{C}^{2}, 0\right)$ the parametrization. We denote by $\left(S^{\mathrm{es}}, 0\right)$ the base space of the semiuniversal equisingular deformation of $(C, 0)$ (in this case, isomorphic to the base space of the semiuniversal equisingular deformation of the parametrization). Note that Theorem 3.1 gives a convergent semiuniversal equisingular deformation (even an algebraic representative) and not only a formal object. $\left(S^{\text {es }}, 0\right)$ is a closed subgerm of the base space $(S, 0)$ of the semiuniversal deformation $(\mathscr{C}, 0) \rightarrow(S, 0)$ of $(C, 0)$. As is well known (cf. [GLS07]), $\left(S^{\mathrm{es}}, 0\right)$ coincides with the $\mu$-constant stratum of $(C, 0)$,

$$
\left(S^{\mathrm{es}}, 0\right)=\left(S^{\mu}, 0\right)=\left(\left\{s \in S \mid \mu\left(\mathscr{C}_{s}\right)=\mu(C, 0)\right\}, 0\right),
$$

where $\mu(C, 0)=\operatorname{dim}_{\mathbb{C}} \mathbb{C}\{x, y\} /\langle\partial f / \partial x, \partial f / \partial y\rangle$ is the Milnor number of $(C, 0), \phi: \mathscr{C} \rightarrow S$ a sufficiently small representative of $(\mathscr{C}, 0) \rightarrow(S, 0)$, and $\mu\left(\mathscr{C}_{s}\right)=\sum_{p \in \mathscr{C}_{s}} \mu\left(\mathscr{C}_{s}, p\right)$ is the total Milnor number of the fibre of $\phi$ over $s$. If $\mu\left(\mathscr{C}_{s}\right)=\mu(C, 0)>0$, then it is also known that there is exactly one singular point $p \in \mathscr{C}_{s}$ (satisfying $\mu\left(\mathscr{C}_{s}, p\right)=\mu\left(\mathscr{C}_{s}\right)$ ), and the restriction of $\phi$ to $\phi^{-1}\left(S^{\mu}\right)$ admits a unique section, picking up the singular point over $s \in S^{\mu}$ (see [Laz73, Tei78]).

Hence, Theorem 4.2 gives a new proof that the $\mu$-constant stratum $\left(S^{\mu}, 0\right)$ is smooth.

(5) In good characteristic, the $\mu$-constant stratum can be generalized to the (strong) equisingularity stratum $S^{\mathrm{es}}=\operatorname{Spec}\left(B^{\mathrm{es}}\right) \subset S=\operatorname{Spec}(B)$. Here, $B$ is the base ring of the semiuniversal deformation $B \rightarrow R_{B}$ of $R$, and $\mathfrak{p} \subset B$ is a prime ideal defining a smooth subscheme such that the restriction $B^{\mathrm{es}}:=B / \mathfrak{p} \rightarrow R_{B} \widehat{\otimes}_{B} B / \mathfrak{p}$ is equisingular. Here $S^{\mathrm{es}}$ is the unique maximal closed subscheme of $S$ having the following universal property: if a strongly equisingular deformation $A \rightarrow R_{A}$ of $R$ is induced from $B \rightarrow R_{B}$ by some map $\psi: B \rightarrow A$, then $\operatorname{Spec}(\psi): \operatorname{Spec}(A) \rightarrow S$ factors through $S^{\mathrm{es}}$. 


\section{EQUISINGULAR DEFORMATIONS IN ARBITRARY CHARACTERISTIC}

(6) In bad characteristic, a 'strongly equisingular stratum' which generalizes the $\mu$-constant stratum, in general does not exist. The situation is as follows: let $\xi^{\mathrm{es}} \in \mathcal{D}$ ef $f_{R}^{\mathrm{es}}\left(B^{\mathrm{es}}\right)$ be a semiuniversal es-deformation, and let $\eta \in \mathcal{D} e f_{R}^{\mathrm{sec}}(B)$ be a semiuniversal deformation with section. Then, by the semiuniversality of $\eta$, there exists a morphism $\psi: B \rightarrow B^{\text {es }}$ in $\mathscr{A}_{K}$, which is unique on the tangent level, such that $\psi \eta \cong \xi^{\text {es }}$. By definition, $\underline{\mathcal{D e f}}_{R}^{\text {es }}$ is a subfunctor of $\underline{\mathcal{D} e f}_{R}$, and we have a unique subspace $T_{R}^{1, \text { es }} \subset T_{R}^{1}$. Since the tangent map of $\psi$ is injective, the dual map $\mathfrak{m}_{B} / \mathfrak{m}_{B}^{2} \rightarrow \mathfrak{m}_{B^{\text {es }}} / \mathfrak{m}_{B^{\text {es }}}^{2}$ is surjective. Thus, $\psi$ induces an isomorphism $B / \operatorname{ker} \psi \cong B^{\text {es }}$, and the deformation over $B / \operatorname{ker} \psi$ induced by $\eta$ is isomorphic to $\xi^{\mathrm{es}}$, hence strongly equisingular semiuniversal.

Thus, $\operatorname{Spec}(B / \operatorname{ker} \psi) \subset \operatorname{Spec}(B)$ could be considered as a (strongly) equisingular stratum. However, it does not have an intrinsic meaning. Indeed, as Example 4.4 shows, $\psi$ is not unique and $\operatorname{Spec}(B / \operatorname{ker} \psi) \subset \operatorname{Spec}(B)$ may vary for different choices of $\psi$ (only the tangent space is fixed). Moreover, the restriction of $\eta$ over the union for different choices is not strongly equisingular.

However, we can define in arbitrary characteristic an intrinsic weak equisingularity stratum in $\operatorname{Spec}(B)$ (see $\S 6$ ) which coincides with the stratum $S^{\text {es }}$ defined in part (5) if the characteristic is good.

Example 4.4. We give now several examples which show that all possible pathologies in bad characteristic do actually occur. Consider the plane curve singularities (in bad characteristic) given by the equation $f$ in the following cases:

(1) $\operatorname{char}(K)=p>0$ and $f=y^{2 p}+x^{2 p+1}+x^{p} y^{p+1}$;

(2) $\operatorname{char}(K)=2$ and $f=y^{4}+x^{6}+x^{7}$;

(3) $\operatorname{char}(K)=p \geqslant 3, l=(p+1) / 2$ and $f=y^{p}-x^{p+2}+x^{l} y^{l}$;

(4) $\operatorname{char}(K)=p>0$ and $f=y\left(y^{p}-x^{p+1}\right)$.

The Tjurina ideal $\langle f, x(\partial f / \partial x), x(\partial f / \partial y), y(\partial f / \partial x), y(\partial f / \partial y)\rangle$ is given by the respective ideals:

(1) $\left\langle x^{2 p+1}, x^{2 p} y, x^{p+1} y^{p}, x^{p} y^{p+1}, y^{2 p}\right\rangle$;

(2) $\left\langle x^{7}, x^{6} y, y^{4}+x^{6}\right\rangle$;

(3) $\left\langle x^{p+2}, 2 x^{p+1} y-l x^{l-1} y^{l+1}, x^{l+1} y^{l-1}, x^{l} y^{l}, y^{p}\right\rangle$;

(4) $\left\langle x^{p+2}-x y^{p}, x^{p+1} y, x^{p} y^{2}, y^{p+1}\right\rangle$.

Thus, a basis for the $K$-vector space $T_{R}^{1, \text { sec }}$ is given by the classes of the monomials with exponents in the set $D$ given by

(1) $D=D_{0} \cup D_{1} \cup D_{2}$ where

$$
\begin{aligned}
& D_{0}=\{(i, j) \mid 0<i+j \leqslant 2 p, j<2 p\}, \\
& D_{1}=\{(i, j) \mid 1<i<p, p+2<j<2 p, i+j>2 p\}, \\
& D_{2}=\{(i, j) \mid p+2<i<2 p, 1<j<p, i+j>2 p\} ;
\end{aligned}
$$

(2) $D=\{(i, j) \mid 0 \leqslant i \leqslant 5,0 \leqslant j \leqslant 3, i+j>0\} \cup\{(6,0)\}$;

(3) $D=D_{0} \cup D_{1} \cup D_{2} \cup\{(p+1,0)\}$ where

$$
\begin{aligned}
& D_{0}=\{(i, j) \mid 0<i+j \leqslant p\}, \\
& D_{1}=\{(i, j) \mid 1<i<l, l<j<p, i+j>p\}, \\
& D_{2}=\{(i, j) \mid l+1<i<p+1,1<j<l-1, i+j>p\} ;
\end{aligned}
$$

(4) $D=\{(i, j) \mid 0<i+j, i<p, j<p+1\} \cup\{(p, 0),(p, 1),(p+1,0)\}$.

In particular, the dimension of $T_{R}^{1, \text { sec }}$ is given by $3 p^{2}+1$ in case (1), 24 in case $(2), 2 l^{2}+(l-1)^{2}$ in case (3), and $p^{2}+p-2$ in case (4). The semiuniversal deformation with section $\eta$ of $R=K[[x, y]] /\langle f\rangle$ 


\section{A. Campillo, G.-M. Greuel and C. Lossen}

is given by

$$
F:=f+\sum_{(i, j) \in D} u_{i, j} x^{i} y^{j}
$$

over the base $S=\operatorname{Spec}(B)$ where $B=K\left[\left[u_{i, j} \mid(i, j) \in D\right]\right]$.

Case (1): The subscheme $S^{\prime} \subset S$ given by the smooth conditions

$$
\begin{aligned}
& u_{i j}=0, \quad(i, j) \in D \backslash\{(p, p),(2 p, 0)\}, \quad \text { and } 2 u_{2 p, 0}-u_{p, p}^{2}=0, \quad \text { if } p \neq 2 ; \\
& u_{i j}=0, \quad(i, j) \in D \backslash\{(4,0)\}, \quad \text { if } p=2
\end{aligned}
$$

can be viewed as an equisingular locus for $\eta$, and one has $\operatorname{dim} S^{\prime}=1+(p-1)(p-2)$. Now, for every $h \in\left\langle u_{i j} \mid(i, j) \in D\right\rangle$ one has the smooth $(p-1)(p-2)$-dimensional subscheme $S_{h}$ of $S^{\prime}$ given by adding, to the equations defining $S^{\prime}$, the following equation

$$
u_{p, p}-2 h^{2}=0, \quad \text { if } p \neq 2 ; \quad u_{4,0}-h^{4}=0, \quad \text { if } p=2 .
$$

Then, for each $h$ the deformation induced by $\eta$ on $S_{h}$ is a semiuniversal deformation for $\underline{\mathcal{D} e f}{ }_{R}^{\text {es }}$, so that $S_{h}=\operatorname{Spec}\left(B / \operatorname{ker} \psi_{h}\right)$ for some $\psi_{h}$ as in Remark 4.3(6). Note that all of the smooth subschemes $S_{h}$ share the same tangent space, namely the subspace $T_{R}^{1, \text { es }}$ of $T_{R}^{1, \text { sec }}$. Also note that $S^{\prime}$ coincides with the Zariski closure of the union of all $S_{h}$.

Case (2): Take any pair $h, h^{\prime} \in\left\langle u_{i j} \mid(i, j) \in D\right\rangle$ and consider the unique $h^{\prime \prime} \in\left\langle u_{i j} \mid(i, j) \in D\right\rangle$ which satisfies the equality

$$
\left(1+h^{\prime \prime 2}\right) u_{42}=h^{\prime 2}+h^{\prime \prime}+\left(1+h^{\prime \prime 2}\right) u_{33} h^{3}+\left(1+h^{\prime \prime}\right)\left(u_{4,3} h^{3}+u_{5,2} h^{2}\right) .
$$

Here $h^{\prime \prime}$ is the solution to an implicit equation in the power series ring $B$. Consider the fivedimensional subscheme $S_{h, h^{\prime}} \subset S$ given by the smooth conditions

$$
\begin{aligned}
& u_{i j}=0, \quad(i, j) \in D \backslash\{(4,0),(4,2),(5,1),(6,0),(3,3),(4,3),(5,2),(5,3)\} \\
& u_{4,0}+h^{4}=0 \\
& u_{5,1}+h^{2} u_{3,3}=0 \\
& u_{6,0}+h^{\prime \prime 2}+u_{5,1} h+u_{4,2} h^{2}+u_{3,3} h^{3}=0 .
\end{aligned}
$$

Again, the deformation induced by $\eta$ on $S_{h, h^{\prime}}$ is semiuniversal for $\underline{\mathcal{D e f}}_{R}^{\text {es }}$, one has $S_{h, h^{\prime}}=$ $\operatorname{Spec}\left(B / \operatorname{ker} \psi_{h, h^{\prime}}\right)$ for some $\psi_{h, h^{\prime}}$, as in Remark 4.3(6), and all $S_{h, h^{\prime}}$ share as tangent space the subspace $T_{R}^{1, \text { es }}$ of $T_{R}^{1, \text { sec }}$. Finally, notice that the Zariski closure of $\bigcup_{h, h^{\prime}} S_{h, h^{\prime}}$ is the seven-dimensional non-smooth subscheme $S^{\prime} \subset S$ given by

$$
\begin{aligned}
& u_{i j}=0,(i, j) \in D \backslash\{(4,0),(4,2),(5,1),(6,0),(3,3),(4,3),(5,2),(5,3)\} \\
& u_{5,1}^{2}+u_{4,0} u_{3,3}^{2}=0 .
\end{aligned}
$$

Case (3): The subscheme $S^{\prime} \subset S$ given by the smooth conditions

$$
u_{i, j}=0, \quad(i, j) \in D, i+j \leqslant p \text { or } i+j=p+1 \text { and } j \leqslant l-1,
$$

can be viewed as the equisingular locus for $\eta$, and one has $\operatorname{dim} S^{\prime}=(l-2)^{2}$. The deformation induced by $\eta$ on $S^{\prime}$ is a semiuniversal deformation for $\underline{\mathcal{D} e}_{R}^{\mathrm{es}}$, therefore $S^{\prime}$ is the only subscheme of type $\operatorname{Spec}(B / \operatorname{ker} \psi)$ for $\psi$ as in Remark 4.3(6).

Case (4): The subscheme $S^{\prime} \subset S$ given by the smooth conditions

$$
u_{i, j}=0, \quad(i, j) \in D, i+j \leqslant p+1,
$$

again, can be viewed as the equisingular locus for $\eta$, and one has $\operatorname{dim} S^{\prime}=\frac{1}{2}(p-1)(p-2)$. The deformation induced by $\eta$ on $S^{\prime}$ is semiuniversal for $\underline{\mathcal{D} e f}_{R}^{\mathrm{es}}$, and the subscheme $S$ is the only one of type $\operatorname{Spec}(B / \operatorname{ker} \psi)$ with $\psi$ as in Remark $4.3(6)$. 


\section{EQUISINGULAR DEFORMATIONS IN ARBITRARY CHARACTERISTIC}

Conclusion. In all four cases the subscheme $S^{\prime}$ will become the weak equisingularity stratum $(\S 6)$.

In case (1) (respectively, case (2)) $S^{\prime}$ is the Zariski closure of infinitely many substrata $S_{h}$ (respectively, $S_{h, h^{\prime}}$ ) which are smooth with the same tangent space. For each $h$ (respectively, $\left(h, h^{\prime}\right)$ ) the restriction of $\eta$ to $S_{h}$ (respectively, $S_{h, h^{\prime}}$ ) is a semiuniversal strongly equisingular deformation of $R$. However, the restriction of $\eta$ to the union of two different strata of $\left\{S_{h}\right\}$ (respectively, $\left\{S_{h, h^{\prime}}\right\}$ ) is not (strongly) equisingular. Hence, an intrinsic largest strong equisingularity stratum in $S^{\prime}$ does not exist. In case (1), $S^{\prime}$ is smooth while in case (2) $S^{\prime}$ is singular.

In cases (3) and (4) $S^{\prime}$ is a strong equisingularity stratum. It exists (and coincides with the weak version) although the characteristic is bad.

\section{Exact sequences of infinitesimal deformations}

In this section, we consider maps between the tangent spaces of the deformation functors introduced so far. We consider additionally deformations of the normalization (without sections) which fix $R$. They are given by morphisms $R_{A} \rightarrow \bar{R}_{A}$ over $A$ such that $R_{A}=R \widehat{\otimes} A$ (and each morphism between them induces the identity on $R_{A}$ ). The corresponding category (respectively, deformation functor) is denoted by $\mathcal{D} e f_{\bar{R} / R}$ (respectively, $\underline{\mathcal{D} e f} \bar{R} / R$ ).

We denote by $T_{R}^{0}(\bar{R}), T_{R}^{0}, T_{\bar{R}}^{0}, T_{\text {Quot }(R)}^{0}$ the $K$-vector space of derivations of $R$ in $\bar{R}, R$ in $R, \bar{R}$ in $\bar{R}$, and $\operatorname{Quot}(R)$ in $\operatorname{Quot}(R)$, respectively. Because all of the considered derivations can be extended to $\operatorname{Quot}(R)$, we can regard $T_{R}^{0}(\bar{R}), T_{R}^{0}, T_{\bar{R}}^{0}$ as vector subspaces of $T_{\mathrm{Quot}(R)}^{0}$.

From the obvious relations among the deformation functors, we deduce linear maps $T_{\bar{R} / R}^{1} \rightarrow$ $T_{\bar{R} \leftarrow R}^{1} \rightarrow T_{R}^{1}$. The elements of $T_{\bar{R} / R}^{1}=\underline{\mathcal{D} e f} \bar{R} / R(K[\varepsilon])$ can be interpreted as derivation classes in the following sense: each deformation of $R \rightarrow \bar{R}$ which fixes $R$ is represented by a deformation $R \otimes_{K} K[\varepsilon] \hookrightarrow \bar{R} \otimes_{K} K[\varepsilon]$, given by an injective morphism $R+\varepsilon R \rightarrow \bar{R}+\varepsilon \bar{R}$ of $K$-algebras mapping an element $g$ of $R+\varepsilon R$ to $g+\varepsilon \partial g$ for some fixed $\partial \in T_{R}^{0}(\bar{R})$. Since two such morphisms define isomorphic deformations if and only if their derivatives are equal modulo $T_{\bar{R}}^{0} \cap T_{R}^{0}(\bar{R})$, we can identify $T_{\bar{R} / R}^{1}$ with the quotient $T_{R}^{0}(\bar{R}) /\left(T_{\bar{R}}^{0} \cap T_{R}^{0}(\bar{R})\right)$. The kernel of the map $T_{\bar{R} / R}^{1} \rightarrow T_{\bar{R} \leftarrow R}^{1}$ consists of the deformation classes determined by derivations in $T_{R}^{0} \subset T_{R}^{0}(\bar{R})$. Thus, it is identified with the $K$-vector space

$$
M_{R}:=T_{R}^{0} /\left(T_{R}^{0} \cap T_{R}^{0}\right) .
$$

Note that, in characteristic zero, we have $M_{R}=0$ as every derivation in $T_{R}^{0}$ can be extended to one in $T_{\bar{R}}^{0}$ (see [Del73]). However, if $\operatorname{char}(K)=p>0$, this is not true. For instance, if $p \nmid q$, the derivative $\partial / \partial y$ is tangent to the curve $\left\{y^{p}+x^{q}=0\right\}$ and the induced derivation is in $T_{R}^{0}$ but not in $T_{\bar{R}}^{0}$.

The involved $K$-vector spaces can also be described in terms of the parametrization of the curve. In fact, denote by $\mathbf{d}=\left(d_{1}, \ldots, d_{r}\right)$ the differential multi-exponent, that is, $d_{i}=\min \left\{\operatorname{ord}_{t_{i}}\left(\dot{x}_{i}\right)\right.$, $\left.\operatorname{ord}_{t}\left(\dot{y}_{i}\right)\right\}$. Then, deformations of the parametrization which give rise to classes in $T_{\bar{R} / R}^{1}$ are precisely those defined by power series $X_{i}, Y_{i} \in A\left[\left[t_{i}\right]\right]$ of type $X_{i}=x_{i}+\varepsilon h_{i} t_{i}^{-d_{i}} \dot{x}_{i}, Y_{i}=y_{i}+\varepsilon h_{i} t_{i}^{-d_{i}} \dot{y}_{i}$ where $h_{i} \in K\left[\left[t_{i}\right]\right]$. Deformations leading to elements in $T_{\bar{R} \leftarrow R}^{1}$ are precisely those given by $X_{i}=x_{i}+\varepsilon a_{i}$, $Y_{i}\left(t_{i}\right)=y_{i}+\varepsilon b_{i}$, where $a_{i}, b_{i} \in K\left[\left[t_{i}\right]\right]$.

Taking into account those deformations leading to trivial deformations for the respective functors, we get the following lemma. 


\section{A. Campillo, G.-M. Greuel and C. Lossen}

Lemma 5.1. We have the following isomorphisms of $K$-vector spaces:

$$
\begin{aligned}
& M_{R} \cong \frac{\mathbf{t}^{-\mathbf{d}} \bar{R} \cdot(\dot{\mathbf{x}}, \dot{\mathbf{y}}) \cap(R \oplus R)}{\bar{R} \cdot(\dot{\mathbf{x}}, \dot{\mathbf{y}}) \cap(R \oplus R)}, \quad T_{\bar{R} / R}^{1} \cong \frac{\mathbf{t}^{-\mathbf{d} \bar{R} \cdot(\dot{\mathbf{x}}, \dot{\mathbf{y}})}}{\bar{R} \cdot(\dot{\mathbf{x}}, \dot{\mathbf{y}})}, \\
& T_{\bar{R} \leftarrow R}^{1} \cong \frac{\bar{R} \oplus \bar{R}}{\bar{R} \cdot(\dot{\mathbf{x}}, \dot{\mathbf{y}})+(R \oplus R)}, \quad T_{R}^{1} \cong R / J,
\end{aligned}
$$

where $J$ is the Jacobian ideal of the curve, that is, the ideal of $R=K[[x, y]] /\langle f\rangle$ generated by the partials $\partial f / \partial x, \partial f / \partial y$. In particular, the vector spaces are all equipped with a natural $R$-module structure.

Here, $\mathbf{t}^{-\mathbf{d}} \bar{R}$ is a short-hand notation for

$$
\bigoplus_{i=1}^{r} t_{i}^{-d_{i}} K\left[\left[t_{i}\right]\right] \subset \operatorname{Quot}(R)=\bigoplus_{i=1}^{r} \operatorname{Quot}\left(\bar{R}_{i}\right)=\bigoplus_{i=1}^{r} K\left(\left(t_{i}\right)\right) .
$$

Note that, in good characteristic, we have $d_{i}=m_{i}-1$.

Altogether, we get an exact sequence of $R$-modules, which are finite-dimensional $K$-vector spaces (see also [Buc81] and [GLS07]).

$$
0 \longrightarrow M_{R} \longrightarrow T_{\bar{R} / R}^{1} \longrightarrow T_{\bar{R} \leftarrow R}^{1} \longrightarrow T_{R}^{1} \longrightarrow R / \bar{R} J \longrightarrow 0 .
$$

All maps are obvious, except for the map $T_{\bar{R} \leftarrow R}^{1} \rightarrow T_{R}^{1}$, which takes the class of $(\mathbf{a}, \mathbf{b}) \in \bar{R} \oplus \bar{R}$ to the class mod $J$ of the element $\mathbf{a}(\partial f / \partial x)+\mathbf{b}(\partial f / \partial y)$. Note that $\bar{R} J$ is an ideal of $R$ as $J$ is contained in the conductor $\mathcal{C}$ of $R$.

For deformations with sections we have an analogous exact sequence of $R$-modules:

$$
0 \longrightarrow M_{R}^{\mathrm{sec}} \longrightarrow T_{\bar{R} / R}^{1, \mathrm{sec}} \longrightarrow T_{\bar{R} \leftarrow R}^{1, \mathrm{sec}} \longrightarrow T_{R}^{1, \mathrm{sec}} \longrightarrow \mathfrak{m} / \overline{\mathfrak{m}} J \longrightarrow 0 .
$$

Here, $\mathfrak{m}$ denotes the maximal ideal of $R, \overline{\mathfrak{m}}$ the Jacobson radical of $\bar{R}$, and

$$
M_{R}^{\mathrm{sec}}:=T_{R}^{0, \mathrm{sec}} /\left(T_{\bar{R}}^{0, \mathrm{sec}} \cap T_{R}^{0, \mathrm{sec}}\right),
$$

where $T_{R}^{0, \text { sec }}=\left\{\partial \in T_{R}^{0} \mid \partial(\mathfrak{m}) \subset \mathfrak{m}\right\}, T_{\bar{R}}^{0, \text { sec }}=\left\{\partial \in T_{\bar{R}}^{0} \mid \partial(\overline{\mathfrak{m}}) \subset \overline{\mathfrak{m}}\right\}$.

Lemma 5.2. We have the following isomorphisms of $R$-modules:

$$
\begin{aligned}
& M_{R}^{\mathrm{sec}} \cong \frac{\mathbf{t}^{-\mathbf{d}+\mathbf{1}} \bar{R} \cdot(\dot{\mathbf{x}}, \dot{\mathbf{y}}) \cap(\mathfrak{m} \oplus \mathfrak{m})}{\overline{\mathfrak{m}} \cdot(\dot{\mathbf{x}}, \dot{\mathbf{y}}) \cap(\mathfrak{m} \oplus \mathfrak{m})}, \quad T_{\bar{R} / R}^{1, \mathrm{sec}} \cong \frac{\mathbf{t}^{-\mathbf{d}+\mathbf{1}} \bar{R} \cdot(\dot{\mathbf{x}}, \dot{\mathbf{y}})}{\overline{\mathfrak{m}} \cdot(\dot{\mathbf{x}}, \dot{\mathbf{y}})}, \\
& T_{\bar{R} \leftarrow R}^{1, \text { sec }} \cong \frac{(\overline{\mathfrak{m}} \oplus \overline{\mathfrak{m}})}{\overline{\mathfrak{m}} \cdot(\dot{\mathbf{x}}, \dot{\mathbf{y}})+(\mathfrak{m} \oplus \mathfrak{m})}, \quad T_{R}^{1, \mathrm{sec}} \cong \mathfrak{m} / \mathfrak{m} J .
\end{aligned}
$$

Remark 5.3. In the description of $M_{R}, M_{R}^{\text {sec }}$ as well as of $T_{\bar{R} / R}^{1}, T_{\bar{R} / R}^{1, \text { sec }}$ in Lemmas 5.1 and 5.2 , each involved derivation $\partial$ is represented by the tuple $(\partial x, \partial y)$ in $R \oplus R$, respectively, in $\bar{R} \oplus \bar{R}$.

From the above exact sequences, we deduce the following lemma.

Lemma 5.4. Let $\delta=\operatorname{dim}_{K} \bar{R} / R$. Then the following hold:

$$
\begin{aligned}
\operatorname{dim}_{K} T_{R}^{1} & =\operatorname{dim}_{K} T_{R}^{1, \mathrm{sec}}-\operatorname{dim}_{K}(J / \mathfrak{m} J)+1, \\
\operatorname{dim}_{K} T_{R}^{1} & =\operatorname{dim}_{K} T_{\bar{R} \leftarrow R}^{1}+\delta+\operatorname{dim}_{K} M_{R}, \\
\operatorname{dim}_{K} T_{R}^{1, \mathrm{sec}} & =\operatorname{dim}_{K} T_{\bar{R} \leftarrow R}^{1, \sec }+\delta+r-1+\operatorname{dim}_{K} M_{R}^{\text {sec }},
\end{aligned}
$$

where $1 \leqslant \operatorname{dim}_{K}(J / \mathfrak{m} J) \leqslant 2$. If $\operatorname{char}(K)=0$, then $\operatorname{dim}_{K} M_{R}=\operatorname{dim}_{K} M_{R}^{\mathrm{sec}}=0$ and, if $R$ is not regular, $\operatorname{dim}_{K}(J / \mathfrak{m} J)=2$. 


\section{EQUISINGULAR DEFORMATIONS IN ARBITRARY CHARACTERISTIC}

Proof. The first equality follows from the definitions. Moreover, we have

$$
\operatorname{dim}_{K} T \frac{1}{R / R}=\operatorname{dim}_{K} T_{\bar{R} / R}^{1, \text { sec }}=|\mathbf{d}|:=d_{1}+\cdots+d_{r} .
$$

From the equality $J \bar{R}=t^{\mathrm{d}} \mathcal{C}$ and the fact that $\operatorname{dim}_{K} \bar{R} / \mathcal{C}=2 \delta$, we get

$$
\operatorname{dim}_{K} R /(J \bar{R})=\delta+|\mathbf{d}|, \quad \operatorname{dim}_{K} \mathfrak{m} /(\overline{\mathfrak{m}} \cdot \bar{R} J)=\delta+r-1+|\mathbf{d}| .
$$

The fact that $\operatorname{dim}_{K}(J / \mathfrak{m} J)=2$ in characteristic zero follows, for example, from [Cas00].

Proposition 5.5. The exact sequence (5.1) induces an exact sequence of $R$-modules

$$
0 \longrightarrow M_{R}^{\mathrm{sec}} \longrightarrow T_{\bar{R} / R}^{1, \mathrm{es}} \longrightarrow T_{\bar{R} \leftarrow R}^{1, \mathrm{es}} \longrightarrow T_{R}^{1, \mathrm{es}} \longrightarrow 0
$$

where $M_{R}^{\mathrm{sec}}=T_{\bar{R} / R}^{1, \mathrm{es}}=0$ if the characteristic of $K$ is good.

Proof. To check the exactness, first note that the map $T_{\bar{R} \leftarrow R}^{1, \text { es }} \rightarrow T_{R}^{1 \text {,es }}$ is surjective by definition of $\underline{D} e f_{R}^{\text {es }}$, and that elements in its kernel are nothing but classes of equisingular deformations keeping $R$ fixed. Second, the image of $M_{R}^{\mathrm{sec}}$ in $T_{\bar{R} / R}^{1, \mathrm{sec}}$ consists of the classes of trivial deformations of the parametrization keeping $R$ fixed. So, $M_{R}^{\text {sec }}$ is contained in $T_{\bar{R} / R}^{1, \mathrm{es}}$.

In good characteristic, a deformation of $\bar{R} \leftarrow P$ given by $X_{i}=x_{i}+\varepsilon h_{i} t_{i}^{-d_{i}+1} \dot{x}_{i}, Y_{i}=y_{i}+$ $\varepsilon h_{i} t_{i}^{-d_{i}+1} \dot{y}_{i}$ is equimultiple along the trivial section if and only if $\operatorname{ord}_{t_{i}}\left(h_{i}\right) \geqslant d_{i}$. Thus, in this case, $T_{\bar{R} / R}^{1, \text { es }}=\{0\}=M_{R}^{\mathrm{sec}}$, and the exact sequence simply states that the map $T_{\bar{R} \leftarrow R}^{1, \text { es }} \rightarrow T_{R}^{1, \text { es }}$ is an isomorphism.

In bad characteristic, however, each term in the sequence of Proposition 5.5 can be nonzero, as the following examples show.

Example 5.6. Let $\operatorname{char}(K)=p>0$.

(1) The irreducible plane curve singularity given by

$$
f=y^{2 p}+x^{2 p+1}+x^{p} y^{p+1}
$$

satisfies $\operatorname{dim}_{K}\left(M_{R}^{\mathrm{sec}}\right)=0, \operatorname{dim}_{K}\left(T_{\bar{R} / R}^{1, \mathrm{es}}\right)=1$. Indeed, it is parametrized by

$$
x(t)=\frac{-t^{2 p}}{1+t^{p+1}}, \quad y(t)=\frac{-t^{2 p+1}}{1+t^{p+1}},
$$

and under the isomorphism of Lemma $5.2, T_{\bar{R} / R}^{1, \text { es }}$ is generated by the class of $(\dot{x}, \dot{y})=\left(t^{3 p} /\left(1+t^{p+1}\right)^{2}\right.$, $\left.-t^{2 p} /\left(1+t^{p+1}\right)^{2}\right)$, however, $M_{R}^{\text {sec }}=0$ as $\dot{x} \notin \mathfrak{m}$. Note that, in this case, $T_{\bar{R} \leftarrow R}^{1, \text { es }}$ and $T_{R}^{1, \text { es }}$ are not isomorphic; in fact, one has $\operatorname{dim}_{K}\left(T_{\bar{R} \leftarrow R}^{1, \mathrm{es}}\right)=1$ and $\operatorname{dim}_{K}\left(T_{R}^{1, \mathrm{es}}\right)=0$.

(2) The irreducible plane curve singularity given by

$$
f=y^{p^{2}}-x^{p^{2}+p}-x^{p^{2}+p+1}=\left(y^{p}-x^{p+1}\right)^{p}-x^{p^{2}+p+1}
$$

satisfies $\operatorname{dim}_{K}\left(M_{R}^{\mathrm{sec}}\right)=\operatorname{dim}_{K}\left(T_{\bar{R} / R}^{1, \mathrm{es}}\right)=2$. Indeed, it is parametrized by

$$
x(t)=t^{p^{2}}, \quad y(t)=t^{p^{2}+p}+t^{p^{2}+p+1},
$$

and under the isomorphism of Lemma $5.2, M_{R}^{\text {sec }}$ is the two-dimensional $K$-vector space generated by the classes of $\left(0, t^{p^{2}}\right)$ and $\left(0, t^{p^{2}+p}\right)$, i.e. by the classes modulo $T_{\bar{R}}^{0, \text { sec }} \cap T_{R}^{0, \text { sec }}$ of the elements of $T_{R}^{0, \text { sec }}$ given by $x(\partial / \partial y)$ and $y(\partial / \partial y)$. Note that, in this case, $T_{\bar{R} \leftarrow R}^{1, \text { es }} \cong T_{R}^{1, \text { es }}$.

(3) The irreducible plane curve singularity

$$
f=y^{p}-x^{p+2}+x^{l} y^{l}
$$




\section{A. Campillo, G.-M. Greuel and C. Lossen}

for $p \geqslant 3$ and $l=\frac{1}{2}(p+1)$ satisfies $\operatorname{dim}_{K} M_{R}^{\text {sec }}=\operatorname{dim}_{K} T_{R}^{1, \text { es }}=0$. Indeed, it is parametrized by

$$
x(t)=\frac{t^{p}}{(1+t)^{l-1}}, \quad y=\frac{t^{p+2}}{(1+t)^{l}}
$$

and, since $d=p$, under the isomorphism of Lemma $5.2, T_{\bar{R} / R}^{1, \text { es }}=0$, as one has $(\dot{x}, \dot{y})=\left((1-l) t^{p} /(1+t)^{l}\right.$, $\left.\left(2 t^{p+1}-l t^{p+2}\right) /(1+t)^{l+1}\right)$. It follows that the deformations of type

$$
\begin{aligned}
& X(t)=x(t)+\varepsilon s t^{-d+1} \dot{x} \\
& Y(t)=y(t)+\varepsilon s t^{-d+1} \dot{y}
\end{aligned}
$$

which are equisingular must satisfy $s \in t^{d} \bar{R}$, so $T_{\bar{R} / R}^{1, \text { es }}=0$. Note that, in this case, one has $T_{\bar{R} \leftarrow R}^{1 \text {,es } \cong}$ $T_{R}^{1, \text { es }}$.

(4) The plane curve singularity with $r \geqslant 2$ branches given by

$$
f=y\left(y-x^{2}\right)\left(y-x^{3}\right) \ldots\left(y-x^{r-1}\right)\left(y^{p}-x^{p+1}\right)
$$

satisfies $\operatorname{dim}_{K} M_{R}^{\text {sec }}=\operatorname{dim}_{K} T_{R}^{1, \text { es }}=0$. Indeed, the differential multi-exponent is given by the $r$-tuple $d=(0, \ldots, 0, p)$, so the elements in $t^{-d+1} \bar{R}(\dot{x}, \dot{y})$ which give rise to equisingular deformations of the parametrization over $K[\varepsilon]$ need to be in $t \bar{R}(\dot{x}, \dot{y})$. This shows $T_{\bar{R} / R}^{1, \text { es }}=0$. Also, in this case, one has $T_{\bar{R} \leftarrow R}^{1, \mathrm{es}} \cong T_{R}^{1, \mathrm{es}}$.

Wahl showed in [Wah74a] that the tangent space to his functor ES is an ideal $I \subset P=K[[x, y]]$, the important equisingularity ideal of $R$. Let us show how this generalizes in our context to arbitrary characteristic.

Let us denote by $\mathcal{D e f}_{R / P}$ the category of deformations of $P \rightarrow R$ inducing the product deformation of $P$, also denoted by (embedded) deformations of $R / P$. The forgetful functor $\operatorname{Def}_{R / P} \rightarrow \mathcal{D e f}_{R}$ is smooth, and we denote by $\mathcal{D} e f_{R / P}^{\text {es }}$ the preimage in $\mathcal{D} e f_{R / P}$ of $\mathcal{D} e f_{R}^{\text {es }}$, and by $\mathcal{D} e f_{R / P \text {, fix }}^{\text {es }}$ the objects in $\mathcal{D} e f_{R / P}^{\mathrm{es}}$ which are equisingular along the trivial section.

Proposition 5.7. The tangent space $\mathcal{D} e f_{R / P}^{\mathrm{es}}(K[\varepsilon])$ of $\mathcal{D} e f_{R / P}^{\mathrm{es}}$,

$$
I^{\mathrm{es}}:=\left\{g \in P \mid f+\varepsilon g \text { defines an element of } \mathcal{D}_{R / P}^{\mathrm{es}}(K[\varepsilon])\right\}
$$

is an ideal containing the Tjurina ideal $\langle f, \partial f / \partial x, \partial f / \partial y\rangle$. Likewise, the tangent space $\mathcal{D} e f_{R / P \text {, fix }}^{\text {es }}$ $(K[\varepsilon])$ of $\mathcal{D} e f_{R / P, \text { fix }}^{\mathrm{es}}$,

$$
I_{\mathrm{fix}}^{\mathrm{es}}:=\left\{g \in P \mid f+\varepsilon g \text { defines an element of } \mathcal{D}_{R / P, \mathrm{fix}}^{\mathrm{es}}(K[\varepsilon])\right\}
$$

is an ideal containing $\langle f\rangle+\mathfrak{m}_{P}\langle\partial f / \partial x, \partial f / \partial y\rangle$.

Moreover, the canonical map $I_{R / P, \mathrm{eix}}^{\mathrm{es}} \rightarrow I_{R / P}^{\mathrm{es}}$ induces an isomorphism

$$
T_{R}^{1, \mathrm{es}} \cong I^{\mathrm{es}} /\langle f, \partial f / \partial x, \partial f / \partial y\rangle \cong I_{\text {fix }}^{\mathrm{es}} /\left(\langle f\rangle+\mathfrak{m}_{P}\langle\partial f / \partial x, \partial f / \partial y\rangle\right) .
$$

Proof. Consider the image of the $R$-module $I \bar{R} \leftarrow P$ (Corollary 3.5) under the map $\bar{R} \oplus \bar{R} \rightarrow R$ given by $(\mathbf{a}, \mathbf{b}) \mapsto \mathbf{a}(\partial f / \partial x)+\mathbf{b}(\partial f / \partial y) ; I_{\text {fix }}^{\text {es }}$ is the preimage in $P$ of this image, hence an ideal in $P$.

A slight modification of the proof of Corollary 3.5 shows that

$$
I_{\bar{R} \leftarrow P}^{\mathrm{es}}:=\left\{\begin{array}{l|l}
(\mathbf{a}, \mathbf{b}) \in \overline{\mathfrak{m}} \oplus \overline{\mathfrak{m}} \mid \begin{array}{l}
\left\{\left(x_{i}\left(t_{i}\right)+\varepsilon a_{i}, y_{i}\left(t_{i}\right)+\varepsilon b_{i}\right) \mid i=1, \ldots, r\right\} \\
\text { defines an es-deformation of } P \rightarrow \bar{R} \\
\text { over } K[\varepsilon] \text { along some sections } \bar{\sigma}, \sigma
\end{array}
\end{array}\right\}
$$

is an $R$-submodule of $\bar{R} \oplus \bar{R}$. Taking the image of this submodule in $R$ under the same map as above, and then the preimage in $P$, gives $I^{\text {es }}$.

Note that $I_{\text {fix }}^{\text {es }} /\left(\langle f\rangle+\mathfrak{m}_{P}\langle\partial f / \partial x, \partial f / \partial y\rangle\right)$ is the tangent space to the image of $\underline{\mathcal{D} e f} \frac{\mathrm{es}}{R} \leftarrow P$ in $\underline{\mathcal{D} e f}{ }_{R}^{\mathrm{sec}}$, which is isomorphic to $T_{R}^{1, \text { es }}$ by the remark after Definition 4.1 . 


\section{EQUISINGULAR DEFORMATIONS IN ARBITRARY CHARACTERISTIC}

\section{Weakly equisingular and weakly trivial deformations}

Our definition of (strongly) equisingular deformation of the equation is the canonical extension of equisingularity to fields of arbitrary characteristic $p$. However, there is a new phenomenon in characteristic $p>0$, which does not appear in characteristic zero. For example, the deformation over $K[[a]]$ given by $y^{p}+a x^{p}+x^{p+1}$ is not trivial, but it becomes trivial after the base change $a \mapsto a^{p}$. Similarly, $y^{2 p}+2 a x^{p} y^{p}+a^{2} x^{2 p}+x^{2 p+1}+x^{p} y^{p+1}$ does not define an equisingular deformation, but after the base change $a \mapsto a^{p}$ it does (see Example 5.6(1)). Since this is a very natural phenomenon in positive characteristic, we introduce the new concepts of weak triviality and weak equisingularity. In this section we just state the main results about weakly equisingular deformations. The proofs (which rely on the results of $\S 7$ ) are deferred to $\S 8$.

We start by considering weakly equisingular deformations which are induced from a fixed deformation of $R$.

For $C \in \mathscr{A}_{K}$, let $\mathscr{A}_{C}$ denote the category of Noetherian complete local $C$-algebras. For the following definition, we fix $C \in \mathscr{A}_{K}$ and an object $\eta \in \mathcal{D e f}_{R}^{\mathrm{sec}}(C)$.

Definition 6.1. A weakly equisingular deformation (wes-deformation) of $R$ based in $\eta=(C \rightarrow$ $\left.R_{C}, \tau\right)$ over $A \in \mathscr{A}_{C}$ is a commutative diagram with Cartesian squares

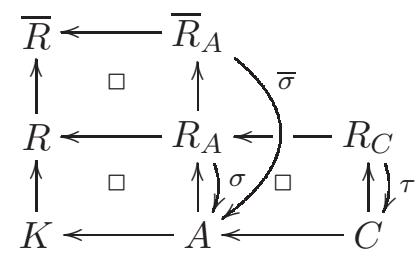

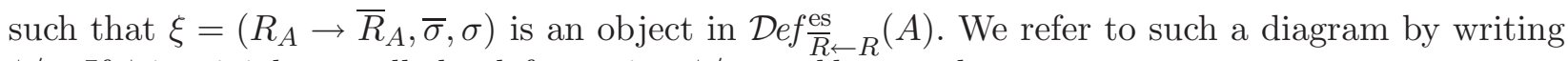
$\xi / \eta$. If $\xi$ is trivial, we call the deformation $\xi / \eta$ weakly trivial.

Here, Def $\frac{\mathrm{es}}{\bar{R} \leftarrow R}$ denotes the category of (strongly) equisingular deformations of the normalization (Definition 2.6). We could have worked with $\mathcal{D} e f \frac{\mathrm{es}}{R \leftarrow P}$ as well, but at this point we prefer deformations of the normalization (which have the induced deformation of $R$ explicitly as part of their data). Recall (Proposition 1.3) that the deformation functors $\underline{\mathcal{D} e f} \overline{\mathrm{es}} \leftarrow R$ and $\underline{\mathcal{D} e f} \bar{R} \leftarrow P$ are isomorphic.

A morphism of wes-deformations based in $\eta$ is given in an obvious way by a commutative diagram (inducing the identity on $\eta$ ). The corresponding category is denoted by $\operatorname{Def}_{R, \eta}^{\text {wes }}$, while $\operatorname{Def} f_{R, \eta}^{\text {wes }}(A)$ denotes the (non-full) subcategory of deformations over $A$ with morphisms being the identity on $A$. If $\psi: A \rightarrow B$ is a morphism in $\mathscr{A}_{C}$, then the induced deformation $\psi \xi$ is an object in $\mathcal{D} e f \frac{\text { es }}{R} \leftarrow R(B)$. Hence, together with $\eta$, it defines an object $\psi(\xi / \eta) \in \operatorname{Def}_{R, \eta}^{\text {wes }}(B)$.

Similarly, we define the category $\mathcal{D} e f_{R, \eta}^{w t r}$ of weakly trivial deformations of $R$ based in $\eta$.

We can now formulate the main results about weakly equisingular deformations of this paper.

Theorem 6.2. Let $\eta=\left(C \rightarrow R_{C}, \tau\right) \in \operatorname{Def}_{R}^{\mathrm{sec}}(C)$ be a fixed deformation with section of $R$. Then the following hold:

(1) There exist an algebra $C_{\eta} \in \mathscr{A}_{C}$ with structure morphism $\Psi_{\eta}: C \rightarrow C_{\eta}$ and an object $\zeta / \eta \in \mathcal{D e f}_{R, \eta}^{\text {wes }}\left(C_{\eta}\right)$ which has the following universal property: if $\xi / \eta \in \operatorname{Def}_{R, \eta}^{\text {wes }}(A), A \in \mathscr{A}_{C}$, then there is a unique morphism $\Psi: C_{\eta} \rightarrow A$ in $\mathscr{A}_{C}$ such that $\Psi(\zeta / \eta)$ is isomorphic to $\xi / \eta$. In particular, $C_{\eta}$ is unique up to a unique isomorphism.

(2) The ideal $I_{\eta}^{\text {wes }}:=\operatorname{ker}\left(\Psi_{\eta}\right) \subset C$ is uniquely determined by $\eta$ and satisfies:

(i) $C_{\eta}^{\text {wes }}=C / I_{\eta}^{\text {wes }} \hookrightarrow C_{\eta}$ is finite;

(ii) for each $z \in C_{\eta}$ there exists a $q=p^{l}$ for some $l \geqslant 0$ such that $z^{q} \in C / I_{\eta}$, where $p=\operatorname{char}(K)$ and $q=1$ if $p$ is good. 


\section{A. Campillo, G.-M. Greuel and C. Lossen}

(3) The construction of $\zeta / \eta$ is functorial in $\eta$ which means the following: let $\eta \in \mathcal{D}$ ef $f_{R}^{\sec }(C)$, $\eta^{\prime} \in \operatorname{Def}_{R}^{\mathrm{sec}}\left(C^{\prime}\right)$ and $\varphi: C \rightarrow C^{\prime}$ a morphism such that $\varphi\left(I_{\eta}^{\text {wes }}\right)=I_{\eta^{\prime}}^{\text {wes }}$ and $\varphi(\eta) \cong \eta^{\prime}$. Let $\zeta / \eta \in \mathcal{D} f_{R, \eta}^{\text {wes }}\left(C_{\eta}\right)$ and $\zeta^{\prime} / \eta^{\prime} \in \mathcal{D}$ ef ${ }_{R, \eta^{\prime}}^{\text {wes }}\left(C_{\eta^{\prime}}\right)$ be the universal objects as in part (1), respectively. Then there exists a morphism $\Psi: C_{\eta} \rightarrow C_{\eta^{\prime}}$ in $\mathscr{A}_{C}$ (where $C_{\eta^{\prime}} \in \mathscr{A}_{C}$ via $C \stackrel{\varphi}{\rightarrow} C^{\prime} \rightarrow C_{\eta^{\prime}}$ ) such that $\Psi(\zeta / \eta) \cong \zeta^{\prime} / \eta^{\prime}$.

(4) Let $\operatorname{Ess}(R)$ denote the set of essential infinitely near points on $R, m_{Q}$ the multiplicity of the strict transform $R_{Q}$ of $R$ at $Q$ and

$$
\operatorname{con}_{R}^{\text {wes }}:=\sum_{Q \in \operatorname{Ess}(R)} \frac{m_{Q}\left(m_{Q}+1\right)}{2}-e f_{R}
$$

where $e f_{R}$ is the number of essential and free points on $R$. Then

$$
\operatorname{dim} C_{\eta}=\operatorname{dim} C_{\eta}^{\text {wes }} \geqslant \operatorname{dim} C-\operatorname{con}{ }_{R}^{\text {wes }} .
$$

(5) If, moreover, $\eta$ is versal in $\mathcal{D} e f_{R}^{\mathrm{sec}}$, then $C_{\eta}$ is a regular local ring, $I_{\eta}^{\text {wes }}$ is a prime ideal and

$$
\operatorname{dim} C_{\eta}^{\text {wes }}=\operatorname{dim} C-\operatorname{con}_{R}^{\text {wes }} .
$$

We call $\zeta / \eta$ from part (1) the universal weakly equisingular deformation of $R$ based in $\eta$ and $\operatorname{con}_{R}^{\text {wes }}$ the number of conditions defining weakly equisingular deformations of $R$.

Definition 6.3. The morphism $\operatorname{Spec}\left(C_{\eta}\right) \rightarrow \operatorname{Spec}(C)$ is finite and we call the image $S_{\eta}^{\text {wes }}:=$ $\operatorname{Spec}\left(C_{\eta}^{\text {wes }}\right) \subset \operatorname{Spec}(C)$ with the scheme structure defined by the prime ideal $I_{\eta}^{\text {es }}$ the weak equisingularity stratum of $\eta$. In good characteristic, strong and weak equisingularity coincide and we call $S_{R}^{\mathrm{wes}}=S_{\eta}^{\mathrm{es}}$ the equisingularity stratum.

If $\eta \in \mathcal{D} e f_{R}^{\mathrm{sec}}\left(B_{R}^{\mathrm{sec}}\right)$ is the semiuniversal deformation of $R$ we write $S_{R}^{\text {wes,sec }}$ instead of $S_{\eta}^{\text {wes }}$. It is a subscheme of the base space $S_{R}^{\mathrm{sec}}:=\operatorname{Spec}\left(B_{R}^{\mathrm{sec}}\right)$ of $\eta$ and called the weak equisingularity stratum of $R$.

We define now weakly equisingular deformations without reference to a given deformation.

Definition 6.4. A deformation $\eta \in \mathcal{D e f}_{R}^{\mathrm{sec}}(C)$ is called a weakly equisingular deformation (with section) of $R$ if there exists an injective $\operatorname{ring} \operatorname{map} \varphi: C \hookrightarrow A$ such that the induced deformation $\varphi \eta \in$ $\mathcal{D} e f_{R}^{\mathrm{sec}}(A)$ is strongly equisingular, that is, an object of $\mathcal{D} e f_{R}^{\mathrm{es}, \mathrm{sec}}(A)$. We denote the corresponding full subcategory of $\mathcal{D} e f_{R}^{\mathrm{sec}}$ by $\mathcal{D} e f_{R}^{\mathrm{wes}, \mathrm{sec}}$ and $\underline{\mathcal{D}}_{R}^{\mathrm{wes}, \mathrm{sec}}$ the functor of weakly equisingular deformations of $R$ with section. If $\varphi \eta$ is trivial we call $\eta$ weakly trivial.

As a corollary of Theorem 6.2 we show that $\mathcal{D} e f_{R}^{\text {wes,sec }}$ has a semiuniversal deformation which can be identified with the restriction of the semiuniversal deformation for $\underline{\mathcal{D e f}}_{R}^{\mathrm{sec}}$ to the weak equisingularity stratum $S_{R}^{\text {wes,sec }} \subset S_{R}^{\text {sec }}$.

Theorem 6.5. Let $\eta \in \mathcal{D e f}_{R}^{\mathrm{sec}}(C)$ be a versal (respectively, semiuniversal) deformation with section of $R$ and $\pi: C \rightarrow C_{\eta}^{\text {wes }}$ the canonical surjection. Then $\pi \eta \in \operatorname{Def}_{R}^{\mathrm{sec}}\left(C_{\eta}^{\text {wes }}\right)$ is a versal (respectively, semiuniversal) weakly equisingular deformation of $R$ with section.

Proof. First let $\eta$ be arbitrary in $\mathcal{D} e f_{R}^{\mathrm{sec}}(C)$ and $\Psi^{\prime}: C \rightarrow C^{\prime}$ a morphism in $\mathscr{A}_{K}$ such that $\Psi^{\prime} \eta \in$ $\mathcal{D} e f_{R}^{\text {wes,sec }}\left(C^{\prime}\right)$. We claim that $\Psi^{\prime}$ factors as $C \stackrel{\pi}{\rightarrow} C_{\eta}^{\text {wes }} \rightarrow C^{\prime}$.

By definition there exists a ring extension $\varphi: C^{\prime} \hookrightarrow A$ such that $\left(\varphi \circ \Psi^{\prime}\right) \eta$ is induced by

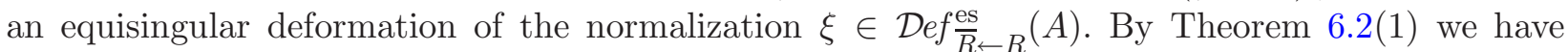
$\xi / \eta \cong \Psi(\zeta / \eta)$ for a unique morphism $\Psi: C_{\eta} \rightarrow A$ with $\zeta / \eta$ the universal object such that the following diagram commutes.

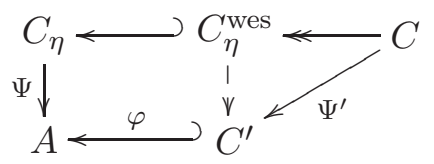




\section{EQUISINGULAR DEFORMATIONS IN ARBITRARY CHARACTERISTIC}

The dotted arrow exists since $\varphi$ is injective, which proves the claim.

Now let $\eta$ be versal and consider two objects $\Theta^{\prime}$ and $\Theta^{\prime \prime}$ in $\mathcal{D} e f_{R}^{\text {wes,sec }}$ over $C^{\prime}$ and $C^{\prime \prime}$, respectively, where $\chi: C^{\prime \prime} \rightarrow C^{\prime}$ is a surjection and $\Theta^{\prime} \cong \chi \Theta^{\prime \prime}$. We have a commutative diagram

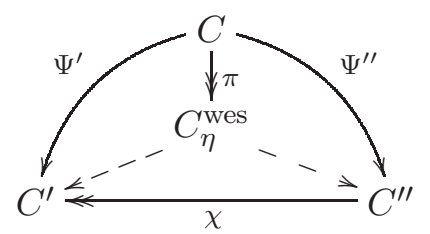

such that $\Psi^{\prime} \eta \cong \Theta^{\prime}, \Psi^{\prime \prime} \eta \cong \Theta^{\prime \prime}$ by the versality property of $\eta$ where the dotted arrows exist by the first part of the proof. This proves the versality of $\pi \eta$.

If $\eta$ is semiuniversal, then the tangent map of $\Psi^{\prime}$ is unique and, hence, so is the tangent map of $C_{\eta}^{\text {wes }} \rightarrow C^{\prime}$. Hence, $\pi \eta$ is semiuniversal too.

Remark 6.6. Let $\eta \in \mathcal{D} e f_{R}^{\mathrm{sec}}\left(B_{R}^{\mathrm{sec}}\right)$ be a semiuniversal deformation of $R$ and $\zeta / \eta \in \mathcal{D} e f_{R, \eta}^{\text {wes }}\left(B_{\eta}\right)$ the universal weakly equisingular deformation of $R$ based in $\eta$.

(1) From Example 4.4 the base space of the semiuniversal weakly equisingular deformation $S_{R}^{\text {wes,sec }} \subset S_{R}^{\mathrm{sec}}=\operatorname{Spec}\left(B_{R}^{\mathrm{sec}}\right)$ is not, in general, smooth in bad characteristic. It is, however, the image of the smooth space $\operatorname{Spec}\left(B_{\eta}\right)$ under the finite and surjective map $\operatorname{Spec}\left(\Psi_{\eta}\right): \operatorname{Spec}\left(B_{\eta}\right) \rightarrow S_{R}^{\text {wes,sec }}$ which is actually a homeomorphism by Theorem $6.2(2)$. In particular, $S_{R}^{\text {wes,sec }}$ is always irreducible of dimension

$$
\operatorname{dim} S_{R}^{\mathrm{wes}, \mathrm{sec}}=\tau^{\mathrm{sec}}(R)-\operatorname{con}^{\mathrm{wes}}(R),
$$

and we can say that the conditions defining the weak equisingularity stratum are independent.

In good characteristic and, as Example 4.4 suggests, also in many cases of bad characteristic, the weak equisingularity stratum $S_{R}^{\text {wes,sec }}$ is smooth.

Here we use the well-known fact that if a map $\varphi: A \rightarrow B$ in $\mathscr{A}_{K}$ is injective then the map $\operatorname{Spec}(\varphi): \operatorname{Spec}(B) \rightarrow \operatorname{Spec}(A)$ is dominant (cf. [GP02, Proposition A.3.8]). If $\varphi$ is finite, then $\operatorname{Spec}(\varphi)$ is closed and, hence, $\operatorname{Spec}(\varphi)$ is surjective.

(2) We comment on the situation for (weakly) trivial deformations: if $\operatorname{char}(K)=0$, then it is known that if a deformation $\eta \in \mathcal{D}$ ef ${ }_{R}(C)$ becomes trivial after some base change $\varphi: C \rightarrow C^{\prime}$, then $\varphi$ factors as $C \stackrel{\pi}{\rightarrow} C^{\text {tr }} \rightarrow C^{\prime}$ where $C^{\text {tr }}$ is a unique factor algebra of $C$ such that $\pi \eta$ is trivial (cf. $[\mathrm{GK} 89$, Lemma 1.4] $)$. Hence, $\operatorname{Spec}\left(C^{\operatorname{tr}}\right) \subset \operatorname{Spec}(C)$ is the unique maximal substratum over which $\eta$ is trivial (and a family is weakly trivial if and only if it is trivial). The proof uses Schlessinger's theory of functors of Artin rings. If $\operatorname{char}(K)>0$, however, we do not know whether Schlessinger' conditions are satisfied and hence we do not know whether unique weakly trivial strata exist.

Everything as above can be formulated for deformations of the parametrization. To do so, fix any object $\zeta=\left(C \rightarrow R_{C} \rightarrow \bar{R}_{C}, \tau, \bar{\tau}\right) \in \mathcal{D}$ ef $\frac{\text { sec }}{R}(C)$ with $C \in \mathscr{A}_{K}$.

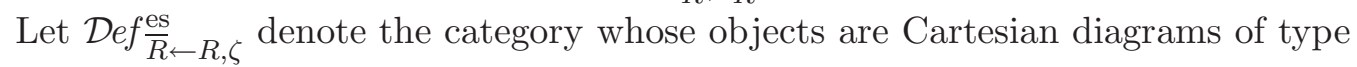

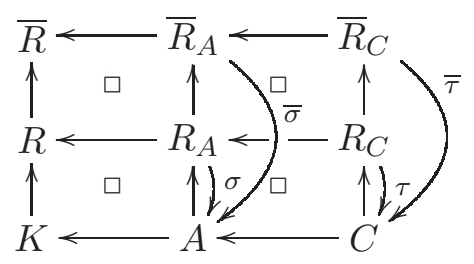

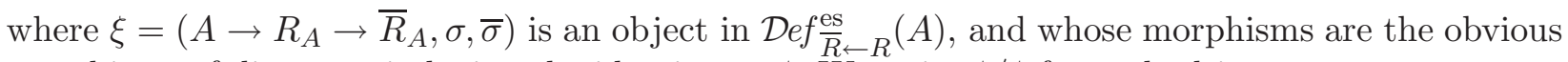
morphisms of diagrams inducing the identity on $\zeta$. We write $\xi / \zeta$ for such objects. 


\section{A. Campillo, G.-M. Greuel and C. Lossen}

For $A \in \mathscr{A}_{C}$ denote by $\operatorname{Def} \frac{\mathrm{es}}{R \leftarrow R, \zeta}(A)$ the (non-full) subcategory of deformations of the parametrization over $A$ with morphisms being the identity on $A$. If $\psi: A \rightarrow B$ is a morphism in $\mathscr{A}_{C}$ and if

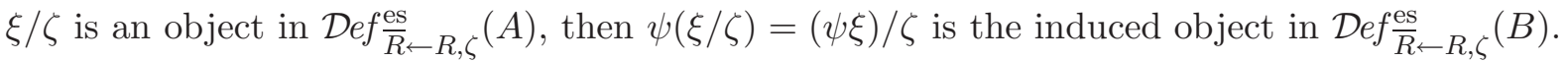

The next theorem is the parametric analogue of Theorem 6.2. Here the situation is, however, simpler.

Theorem 6.7. For each deformation $\zeta=\left(C \rightarrow R_{C} \rightarrow \bar{R}_{C}, \tau, \bar{\tau}\right)$ in $\mathcal{D}$ ef $\frac{\mathrm{sec}}{\bar{R} \leftarrow R}$ there is a universal object $\xi / \zeta$ for the category $\mathcal{D} e f_{\bar{R} \leftarrow R, \zeta}^{\mathrm{es}}$ defined over an algebra $C_{\zeta} \in \mathscr{A}_{C}$ (with structure morphism $\left.\psi_{\zeta}: C \rightarrow C_{\zeta}\right)$ which is functorial in $\zeta$ and has the following properties:

(1) We have $C_{\zeta}=C_{\zeta}^{\mathrm{es}}:=C / I_{\zeta}^{\mathrm{es}}$, where $I_{\zeta}^{\mathrm{es}}=\operatorname{ker} \psi_{\zeta}$.

(2) The codimension of $\operatorname{Spec}\left(C_{\zeta}^{\mathrm{es}}\right) \subset \operatorname{Spec}(C)$ satisfies

$$
\operatorname{dim} C-\operatorname{dim} C_{\zeta}^{\mathrm{es}} \leqslant \operatorname{con} \frac{\mathrm{es}}{\bar{R} \leftarrow P}:=\sum_{Q \in \operatorname{Ess}(R)} m_{Q}-e f_{R}-(r-1),
$$

where $\operatorname{Ess}(R)$ is the set of essential infinitely near points of $R, m_{Q}$ is the multiplicity of the strict transform $R_{Q}$ of $R$ at $Q, e f_{R}$ is the number of free essential points of $R$ and $r$ the number of branches of $R$.

(3) If $\zeta$ is a versal deformation for $\underline{\mathcal{D} e f} \frac{\mathrm{sec}}{\bar{R} \leftarrow R}$, then $C_{\zeta}^{\mathrm{es}}$ is smooth and satisfies

$$
\operatorname{dim} C-\operatorname{dim} C_{\zeta}^{\mathrm{es}}=\operatorname{con} \frac{\mathrm{es}}{R}{ }^{\prime} .
$$

Moreover, the induced deformation $\zeta^{\mathrm{es}}$ of $\zeta$ on $C_{\zeta}^{\mathrm{es}}$ is a versal deformation for the functor

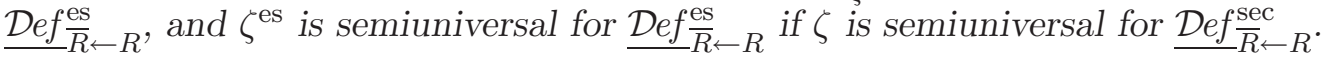

Again, universal in the statement means that if $\xi^{\prime} / \zeta$ is an object in $\operatorname{Def} \frac{\mathrm{es}}{R \leftarrow R, \zeta}(A)$, then there is a unique morphism $\psi: C_{\zeta}^{\text {es }} \rightarrow A$ such that $\psi(\xi / \zeta) \cong \xi^{\prime} / \zeta$. In particular, $C_{\zeta}^{\text {es }}$ is unique up to isomorphism, and it is given by $I_{\zeta}^{\mathrm{es}}$ which is a uniquely defined ideal of $C$, depending functorially on $\zeta$. We call the subscheme $S_{\zeta}^{\mathrm{es}}:=\operatorname{Spec}\left(C_{\zeta}^{\mathrm{es}}\right)=\operatorname{Spec}\left(C / I_{\zeta}^{\mathrm{es}}\right)$ the equisingularity stratum of $\zeta$.

Note that in the parametric case, it is not necessary to consider the analogue of weak equisingular deformations since the universal object for it is the subscheme $S_{\zeta}^{\mathrm{es}}$ of $\operatorname{Spec}(C)$. That is, if a deformation of the parametrization over $A$ becomes equisingular over $B$ after a finite base change $A \hookrightarrow B$, then it was already equisingular before over $A$. This corresponds to the fact that in Theorem 6.7(1) no base change $C_{\zeta}^{\mathrm{es}} \hookrightarrow C_{\zeta}$ as in Theorem 6.2(2)(i) is required.

Sections 7 and 8 will provide proofs for Theorems 6.2 and 6.7 .

\section{Equipolygonal deformations}

In this section, we introduce equipolygonal deformations for embedded plane curve singularities as well as for their parametrizations. Such deformations are auxiliary tools for describing equisingular strata. We also show the relationship between equipolygonal and equisingular deformations.

Throughout the following, we consider a fixed (embedded) plane curve singularity $P \rightarrow R=$ $P /\langle f\rangle$.

\subsection{Equipolygonal deformations of the equation}

Let $Q$ be an infinitely near point of $P$ on $R$, and let $Q \rightarrow R_{Q}=Q /\langle g\rangle$ be the (embedded) strict transform of $R$ at $Q$. If $Q \neq P$, the reduced total transform of $R$ at $Q$ consists of either one or two additional smooth exceptional branches, depending on whether $Q$ is a free point or a satellite point. Namely, the branch $Q \rightarrow E_{Q}$ given by the exceptional divisor of the blowing-up creating $Q$ and, in the satellite case, another exceptional branch $Q \rightarrow D_{Q}$ which is the strict transform of $E_{P^{\prime}}$ for some 


\section{EQUISINGULAR DEFORMATIONS IN ARBITRARY CHARACTERISTIC}

infinitely near point $P^{\prime}$ of $P$ (such that $Q$ is infinitely near to $P^{\prime}$ ). We denote by $Q \rightarrow H_{Q}$ the curve singularity consisting of the exceptional branches at $Q$ (for $Q=P, H_{Q}$ is defined to be the zero ring). We set $\mathcal{P}_{P, R}:=\{P\}$ and, for $Q \neq P$,

$$
\mathcal{P}_{Q, R}:=\left\{\text { infinitely near points of } Q \text { on } R \text { which are on } H_{Q}\right\} .
$$

Moreover, we define $e=e_{Q, R}$ (respectively, $d=d_{Q, R}$ ) to be the number of points in $\mathcal{P}_{Q, R}$ which are on $E_{Q}$ (respectively, on $D_{Q}$ ). If $E_{Q}$ (respectively, $D_{Q}$ ) does not exist, then we set $e=1$ (respectively, $d=1)$

We say that two elements $u, v \in \mathfrak{m}_{Q} \subset Q$ are adapted to $H_{Q}$ (or adapted coordinates of $Q$ ) if $Q=K[[u, v]]$ and if all points in $\mathcal{P}_{Q, R}$ are on $Q /\langle u v\rangle$. That is, up to a permutation of $u$, $v$, we have intersection multiplicities $i\left(E_{Q}, Q /\langle u\rangle\right) \geqslant e$ and $i\left(D_{Q}, Q /\langle v\rangle\right) \geqslant d$ (if $E_{Q}, D_{Q}$ exist). In this case, we say that $u$ is adapted to $E_{Q}$ and $v$ is adapted to $D_{Q}$. In particular, if $E_{Q}=Q /\langle u\rangle$ and $D_{Q}=Q /\langle v\rangle$, then $u$ is adapted to $E_{Q}$ and $v$ is adapted to $D_{Q}$. If $D_{Q}$ does not exist, we call each element $v \in \mathfrak{m}_{Q}$ which is transversal to $u$ adapted to $D_{Q}$. For $Q=P$, any two elements $u, v$ which generate $\mathfrak{m}_{Q}$ are adapted to $H_{Q}$.

We call $u, v \in \mathfrak{m}_{Q}$ generic adapted elements if the set of infinitely near points of $R$ on $Q /\langle u v\rangle$ coincides with $\mathcal{P}_{P, R}$. In other words, two adapted elements $u, v$ are generic adapted if the intersection multiplicity of $Q /\langle u v\rangle$ with $R_{Q}$ is minimal, that is, $\operatorname{ord}_{u} g(u, 0)=i\left(E_{Q}, R_{Q}\right)$ and $\operatorname{ord}_{v} g(0, v)=$ $i\left(D_{Q}, R_{Q}\right)$.

Up to a permutation of $u$ and $v$, the following objects depend only on $R$ and $Q$ (but not on the choice of adapted elements $u, v \in Q$ ).

(1) The Newton polygon $N=N_{Q, R}$ of $g$ with respect to generic adapted elements.

(2) The ideal $I^{\mathrm{ep}}=I_{R_{Q}}^{\mathrm{ep}} \subset Q$ generated by the monomials with exponents in $N+\mathbb{Z}_{\geqslant 0}^{2}$.

(3) The adapted Jacobian ideal $J_{Q, R} \subset Q$ generated by $g, u(\partial g / \partial u), u^{e}(\partial g / \partial v), v^{d}(\partial g / \partial u)$, $v(\partial g / \partial v)$ (assuming that $E_{Q}=Q /\langle u\rangle$ and $D_{Q}=Q /\langle v\rangle$ ). We have $J_{Q, R} \subset I^{\mathrm{ep}}$.

(4) The finite-dimensional $K$-vector space $T^{\mathrm{ep}}=T_{Q, R}^{\mathrm{ep}}=I^{\mathrm{ep}} / J_{Q, R}$.

It is easy to see that $J_{Q, R}$ is the tangent space to the group of adapted automorphisms of $Q$. Here, an automorphism $\psi$ of $Q$ is called adapted if $\psi(u) \in\left\langle u, v^{e}\right\rangle, \psi(v) \in\left\langle u^{d}, v\right\rangle$ (for any adapted $u, v$ with $u$ adapted to $E_{Q}$ and $v$ adapted to $D_{Q}$ ). Note that adapted automorphisms map adapted elements to adapted elements.

Definition 7.1. Let $\eta=\left(Q_{A} \rightarrow \bar{H}_{Q, A}, \bar{\sigma}, \sigma\right) \in \mathcal{D} e f \bar{H}_{Q \leftarrow Q}^{\text {sec }}(A)$ be a deformation with section of the parametrization of $H_{Q}$, and let $\xi=\left(A \rightarrow R_{Q, A}, \sigma^{\prime}\right)$ define an object of $\mathcal{D}_{R_{Q}}^{\text {sec }}(A)$. Then we say that $\xi$ is adapted to $\eta$ if $\xi$ fits into a commutative diagram

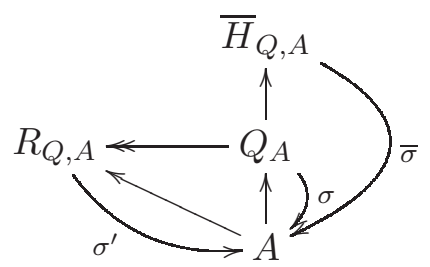

that is, $\eta$ and $\xi$ are deformations with basically the same section, namely $\sigma=Q_{A} \rightarrow R_{Q, A} \stackrel{\sigma^{\prime}}{\longrightarrow} A$.

Let $u, v \in Q$ be generic adapted elements, and let $U, V \in I_{\sigma} \subset Q_{A}$ be such that $u \equiv U$ $\bmod \mathfrak{m}_{A}$ and $v \equiv V \bmod \mathfrak{m}_{A}$. Then Nakayama's lemma implies that $U, V$ generate $I_{\sigma} / I_{\sigma}^{2}$, thus, $Q_{A}=A[[U, V]]$ and $I_{\sigma}=\langle U, V\rangle$. 


\section{A. Campillo, G.-M. Greuel and C. Lossen}

We call $U, V$ adapted to $\eta$ if $\operatorname{ord}_{U} E(U, 0)=e$ and $\operatorname{ord}_{V} D(0, V)=d$. Here, $E(U, V) \in A[[U, V]]$ (respectively, $D(U, V) \in A[[U, V]])$ is the equation for the deformation of $E_{Q}$ (respectively, $D_{Q}$ ) induced by $\eta$.

Definition 7.2. Let $\eta=\left(\phi_{A}, \bar{\sigma}, \sigma\right) \in \mathcal{D} e f_{\bar{H}_{Q} \leftarrow Q}^{\mathrm{sec}}(A)$, and let $\xi \in \mathcal{D e f}_{R_{Q}}^{\mathrm{sec}}(A)$ and $U, V \in I_{\sigma}$ be adapted to $\eta$. Moreover, let $G(U, V) \in A[[U, V]]$ be an equation for $\xi$. Then $\xi$ is called

(1) equiadapted if $\operatorname{ord}_{U} G(U, 0)=\operatorname{ord}_{u} g(u, 0), \operatorname{ord}_{V} G(0, V)=\operatorname{ord}_{v} g(0, v)$;

(2) equipolygonal if $G(U, V) \in I_{R_{Q}}^{\mathrm{ep}} A[[U, V]]$.

Note that this definition is independent of the choice of the (generic) adapted elements $u, v, U, V$.

We write $\mathcal{D} e f_{R_{Q}}^{\text {ep }}$ (respectively, $\underline{\mathcal{D} e f}_{R_{Q}}^{\text {ep }}$ ) for the category (respectively, the functor of adapted isomorphism classes) of equipolygonal deformations of $R_{Q}$. The vector space $T^{\mathrm{ep}}$ can then be identified with the tangent space to $\underline{\mathcal{D e f}}_{R_{Q}}^{\mathrm{ep}}$.

For $Q=P$, equiadapted and equipolygonal deformations of $R_{Q}$ are nothing but equimultiple deformations of the equation (along the section prescribed by $\eta$ ). If $Q$ is arbitrary, equiadapted deformations preserve the points of intersection of the Newton polygon $N_{Q, R}$ with the $u$ - and $v$-axes, while equipolygonal deformations preserve the Newton polygon (for generic adapted coordinates).

For each deformation $\xi \in \mathcal{D e f}_{R_{Q}}^{\mathrm{ep}}(A)$, there is a well-defined Kodaira-Spencer map $\Psi=\Psi_{\xi}$ : $T_{A} \rightarrow T^{\mathrm{ep}}$, where $T_{A}=\operatorname{Der}_{K}(A, K)=\operatorname{Hom}(A, K[\varepsilon])$ is the Zariski-tangent space to $A$. For $G \in$ $I^{\mathrm{ep}} A[[U, V]]$ inducing $\xi$, the map $\Psi$ takes a (local) homomorphism $\delta: A \rightarrow K[\varepsilon]$ to $[h] \in T^{\mathrm{ep}}=$ $I^{\mathrm{ep}} / J_{Q, R}$, where $g+\varepsilon h \in K[\varepsilon][[u, v]]$ defines $\delta \xi \in \mathcal{D}_{R_{Q}}^{\mathrm{ep}}(K[\varepsilon])$.

A deformation $\xi \in \mathcal{D} e f_{R_{Q}}^{\text {ep }}(A)$ is called equipolygonal versal (or ep-versal) if the corresponding Kodaira-Spencer map $\Psi_{\xi}$ is surjective. If $\Psi_{\xi}$ is an isomorphism, we call $\xi$ equipolygonal semiuniversal (or ep-semiuniversal).

Proposition 7.3. Let $h_{1}, \ldots, h_{s} \in I_{R_{Q}}^{\mathrm{ep}}$ represent a basis (respectively, a system of generators) of $T_{Q, R}^{\mathrm{ep}}$. Then, with $T_{1}, \ldots, T_{s}$ new variables and $\mathbf{T}=\left(T_{1}, \ldots, T_{s}\right)$,

$$
G=g+\sum_{i=1}^{s} T_{i} h_{i} \in K[[\mathbf{T}]][[u, v]]
$$

defines an ep-semiuniversal (respectively, ep-versal) deformation of $Q /\langle g\rangle$.

In particular, each ep-versal deformation of $R_{Q}$ has a smooth base space.

Proof. Since $\left\{\partial / \partial T_{i} \mid i=1, \ldots, r\right\}$ is a basis of $T_{K[[\mathbf{T}]]}$ and since the Kodaira-Spencer map for $G$ maps $\partial / \partial T_{i}$ to $h_{i}$, the statement is almost a tautology.

Remark 7.4. We introduced the notion of equipolygonal (semiuni)versal at this stage only in order to have a convenient notation. The fact that this notion is equivalent to the usual definition of (semiuni)versality for the functor $\underline{\mathcal{D} e f}_{R_{Q}}^{\mathrm{ep}}$ is by no means trivial and follows from the results of $\S 8$.

We generalize the notions from above to multicurves (respectively, curve diagrams).

Definition 7.5. A curve diagram is a finite list $\mathscr{C}$ of infinitely near points of $P$ on $R$ (repetitions are allowed) together with, for each $Q \in \mathscr{C}$ :

(1) the set of exceptional branches $E_{Q}, D_{Q}$ (if these exist); and

(2) a non-exceptional curve $Q \rightarrow Q /\langle g\rangle$ (that is, a curve without exceptional branches) 
such that the following holds: if $Q \rightarrow Q^{\prime}$ is a formal blow-up among $Q, Q^{\prime} \in \mathscr{C}$, then the nonexceptional curve $Q^{\prime} \rightarrow Q^{\prime} /\left\langle g^{\prime}\right\rangle$ at $Q^{\prime}$ is the strict transform of $Q /\langle g\rangle$ under the formal blow-up. We denote such a curve diagram by $(\mathscr{C}, \mathscr{G})$, where $\mathscr{G}=(Q /\langle g\rangle)_{Q \in \mathscr{C}}$.

If none of the points in $\mathscr{C}$ are consecutive, we also refer to a curve diagram as a multicurve.

Example 7.6. Let $g \in Q$ decompose into $s$ tangential components, that is, $g=g_{1} \cdots \cdots g_{s}$, where the $g_{i}$ are unitangential and have pairwise different tangent directions. Then the multicurve of tangential components of $Q /\langle g\rangle$ is given by the list $\mathscr{C}=(Q, \ldots, Q), Q$ repeated $s$ times, together with, for the $j$ th entry, the set of exceptional branches $E_{Q}, D_{Q}$ (if these exist), and the curve $Q \rightarrow Q /\left\langle g_{j}\right\rangle$.

Definition 7.7. An equipolygonal deformation of a curve diagram $(\mathscr{C}, \mathscr{G})$ over $A$ is a list of objects in $\mathcal{D e f}_{H_{Q} \leftarrow Q}^{\mathrm{sec}}(A), Q \in \mathscr{C}$, and a list of equipolygonal deformations of $Q /\langle g\rangle, Q \in \mathscr{C}, Q /\langle g\rangle \in \mathscr{G}$, adapted to the given deformations of $E_{Q}, D_{Q}$, such that the following holds. If $Q \rightarrow Q^{\prime}$ is a formal blow-up of points in $\mathscr{C}$ and if the equipolygonal deformation of $Q /\langle g\rangle$ is defined by $G \in Q_{A}$ and the section $\sigma: A \rightarrow Q_{A}$, then:

(1) the equipolygonal deformation of $Q^{\prime} /\left\langle g^{\prime}\right\rangle$ is given by the strict transform $G^{\prime}$ of $G$ under the formal blow-up of $I_{\sigma}$ and a section $\sigma^{\prime}: A \rightarrow Q^{\prime}$ which is compatible with $\sigma$;

(2) the given deformation of $E_{Q^{\prime}}$ is the exceptional divisor of the formal blow-up of $I_{\sigma}$;

(3) if $Q^{\prime}$ is satellite, then the given deformation of $D_{Q^{\prime}}$ is the strict transform of the given deformation of the exceptional branch at $Q$ whose strict transform at $Q^{\prime}$ is $D_{Q^{\prime}}$.

For a curve diagram $(\mathscr{C}, \mathscr{G})$, we introduce $\mathbf{T}^{\mathrm{ep}}=\bigoplus_{Q \in \mathscr{C}} T_{Q, Q /\langle g\rangle}^{\mathrm{ep}}$. Further, for each equipolygonal deformation of $(\mathscr{C}, \mathscr{G})$ over $A$, we consider the Kodaira-Spencer map $T_{A} \rightarrow \mathbf{T}^{\mathrm{ep}}$ given componentwise as above. An equipolygonal deformation of a curve diagram is called ep-versal if the Kodaira-Spencer map is surjective.

Proposition 7.8. Let $g=g_{1} \cdots g_{s}$ be the decomposition of $g \in Q$ into tangential components, and assume that $G \in Q_{A}$ defines an equipolygonal deformation of $Q /\langle g\rangle$, then there exists a unique factorization $G=G_{1} \cdots G_{s}$ such that the following hold:

(1) $G_{j}$ defines an equipolygonal deformation of $g_{j}, j=1, \ldots, s$;

(2) if $G$ defines an equipolygonal versal deformation $\xi$ of $Q /\langle g\rangle$, then $\left(G_{1}, \ldots, G_{s}\right)$ defines an equipolygonal versal deformation of the multicurve of tangential components of $Q /\langle g\rangle$.

Proof. First, assume that $\mathcal{P}=\mathcal{P}_{Q, R}$ consists of just the point $P$, that is, $e=d=1$. In this case, since the coordinates are generic, equipolygonal means nothing but equimultiple. We may assume that $u, v \in Q$ are adapted elements and $g$ is a Weierstraß polynomial, $g \in K[[u]][v]$, of degree $m$ equal to the multiplicity of $g$. Then, up to a unit, $G \in A[[U]][V]$ is also a Weierstraß polynomial of degree $m$. The formal transformation $(U, V) \rightarrow\left(U, V^{\prime}\right)$ given by $U \mapsto U, V \mapsto V^{\prime} U$ leads to $g=u^{m} g^{\prime}, G=U^{m} G^{\prime}$, with $g^{\prime} \in K[[u]]\left[v^{\prime}\right]$ (respectively, $G^{\prime} \in A[[U]]\left[V^{\prime}\right]$ ) polynomials of degree $m$ in $v^{\prime}$ (respectively, $V^{\prime}$ ). On the other hand, the factors $g_{i}$ of $g$ can also be assumed to be Weierstraß polynomials, giving rise to transforms $g_{i}^{\prime}$ such that $g^{\prime}=g_{1}^{\prime} \cdots \cdot g_{s}^{\prime}$. Now, the residues modulo $u K\left[\left[u, v^{\prime}\right]\right]$ of the $g_{i}^{\prime}$ are relative prime polynomials in $K\left[v^{\prime}\right]$. So, Hensel's lemma provides us with a factorization $G^{\prime}=G_{1}^{\prime} \cdots G_{s}^{\prime}$, where $G_{i}^{\prime} \in A[[U]]\left[V^{\prime}\right]$ defines a deformation of $Q^{\prime} /\left\langle g_{i}^{\prime}\right\rangle$. Since the $G_{i}^{\prime}$ are polynomials, we may apply the backward transformation, giving rise to the required factorization $G=G_{1} \cdots G_{s}$, with $G_{i}$ defining an equimultiple deformation of $Q /\left\langle g_{i}\right\rangle$ at $P$. The uniqueness of the factorization follows from the uniqueness of Hensel's lifting.

For a general $\mathcal{P}$, first factorize $G=G_{1} \cdots G_{s}$ as equimultiple deformation in terms of tangential components according to the previous step. Now, for those components $Q /\left\langle g_{j}\right\rangle$ which are not tangential to one of the coordinate curves $Q /\langle v\rangle$ or $Q /\langle u\rangle$ (with $u, v$ adapted to $H_{Q}$ ), the deformation given by $G_{j}$ is an equipolygonal deformation as it is equimultiple. If some component $Q /\left\langle g_{j}\right\rangle$ 


\section{A. Campillo, G.-M. Greuel and C. Lossen}

is tangential to $Q /\langle v\rangle$ (respectively to $Q /\langle u\rangle$ ), then the constance of the Newton polygon for $G$ (condition (2) of Definition 7.2) implies the constance of the Newton polygon $N_{i}$ for $G_{i}$, as one can easily deduce from the factorization of $G$. Thus, each $G_{i}$ defines an equipolygonal deformation of $Q /\left\langle g_{j}\right\rangle$, which proves part (1).

For part (2), we have to prove the surjectivity of the Kodaira-Spencer map of the multicurve, $\left(\Psi_{1}, \ldots, \Psi_{s}\right): T_{A} \rightarrow \mathbf{T}^{\mathrm{ep}}$, where $\Psi_{j}: T_{A} \rightarrow T_{Q, Q /\left\langle g_{j}\right\rangle}^{\mathrm{ep}}$ is the Kodaira-Spencer map for the equipolygonal deformation $\xi_{j}$ of $Q /\left\langle g_{j}\right\rangle$ defined by $G_{j}$. To do so, take elements $a_{j} \in I_{Q /\left\langle g_{j}\right\rangle}^{\mathrm{ep}}$ and consider $a=\sum_{j=1}^{s} a_{j} h_{j} \in I_{Q /\langle g\rangle}^{\mathrm{ep}}$, where $h_{j}=g / g_{j}, j=1, \ldots, s$. Since $G$ defines an ep-versal deformation, there exists some $\delta \in T_{A}$ such that $\Psi_{\xi}(\delta)=[a] \in T_{Q, Q /\langle g\rangle}^{\mathrm{ep}}$. We claim that $\Psi_{j}(\delta)=\left[a_{j}\right]$.

To show this claim, let $\rho \in J_{Q, Q /\langle g\rangle}$ be such that the induced deformation $\delta \xi$ be given by $g+\varepsilon(a+\rho)$. Since $g=g_{j} h_{j}$, we may write $\rho$ as $\rho=\rho_{j}^{\prime} h_{j}+b_{j} g_{j}$ for some $\rho_{j}^{\prime} \in J_{Q, Q /\left\langle g_{j}\right\rangle}$ and $b_{j} \in P$ (by definition of the adapted Jacobian ideal). On the other hand, if the induced deformations $\delta \xi_{j}$ are given by $g_{j}+\varepsilon a_{j}^{\prime}$, where $a_{j}^{\prime} \in K[[u, v]]$, then the equality $G=G_{1} \cdots G_{s}$ implies $a+\rho=\sum_{j=1}^{s} a_{j}^{\prime} h_{j}$. Together with the above, we get $\left(a_{j}^{\prime}-a_{j}-\rho_{j}^{\prime}\right) h_{j} \in\left\langle g_{j}\right\rangle \subset K[[u, v]]$ (note that $h_{i}, i \neq j$, is divisible by $g_{j}$ ). Since $h_{j}$ has no common divisor with $g_{j}$, this shows that $a_{j}^{\prime}-a_{j} \in J_{Q, Q /\left\langle g_{j}\right\rangle}$ as required.

Another important example of curve diagrams are blow-up diagrams of non-exceptional curves: let $Q$ be an infinitely near point of $P$ on $R$, and let $Q \rightarrow Q /\langle g\rangle$ be a non-exceptional curve. Then the blow-up diagram $\mathscr{R}_{Q, g}=(\mathscr{C}, \mathscr{G})$ of $Q \rightarrow Q /\langle g\rangle$ is defined as follows: the entries of the list $\mathscr{C}$ are $Q$ and each infinitely near point $Q^{\prime}$ on $R$ in the first neighbourhood of $Q$ (no repetition). The list of non-exceptional curves $\mathscr{G}$ consists of $Q \rightarrow Q /\langle g\rangle$ and its strict transforms $Q^{\prime} \rightarrow Q^{\prime} /\left\langle g^{\prime}\right\rangle$ under the formal blow-up $Q \rightarrow Q^{\prime}$.

The following lemma provides us with necessary conditions for an equipolygonal deformation of $Q \rightarrow Q /\langle g\rangle$ to lift to an equipolygonal deformation of the blow-up diagram.

Lemma 7.9. Let $Q /\langle g\rangle$ be unitangential, let $U, V \in Q_{A}$ be adapted elements such that $u \equiv U$ $\bmod \mathfrak{m}_{A}$ is transversal to $g$, and let $G \in A[[U, V]]$ define an equipolygonal deformation of $Q \rightarrow Q /\langle g\rangle$ along the trivial section. Let $Q^{\prime}$ be an infinitely near point on $R$ in the first neighbourhood of $Q$ corresponding to the linear factor $v+\alpha u, \alpha \in K$, of the tangent cone of $g$. Finally, let $\eta$ be the deformation of $E_{Q^{\prime}}$ given by the exceptional divisor of the formal blow-up of $I_{\sigma}=\langle U, V\rangle$ and the section $\sigma^{\prime}$. Then the following are equivalent:

(i) the strict transform $G^{\prime} \in A\left[\left[U, V^{\prime}\right]\right], V^{\prime}=V / U+\alpha$, of $G$ defines a deformation which is equiadapted to $\eta$

(ii) $G=c(V+a U+\alpha U)^{m}+($ terms of order $>m$ in $U, V)$, for some $a \in \mathfrak{m}_{A}, c \in A^{*}$, and $I_{\sigma^{\prime}}=\left\langle U, V^{\prime}+a\right\rangle$.

Moreover, if condition (ii) holds, $a$ is uniquely determined unless $A$ is non-reduced and the characteristic of $K$ is a divisor of the multiplicity $m$ of $g$.

Proof. Since $\sigma$ is compatible with $\sigma^{\prime}$, we have $I_{\sigma^{\prime}}=\left\langle U, V^{\prime}+a\right\rangle$. As equiadapted implies equimultiple, we may assume that the $m$-jet $L$ of $G$ is a homogeneous polynomial of degree $m$. Then the strict transform $G^{\prime}$ satisfies $G^{\prime}\left(U, V^{\prime}\right) \equiv L\left(1, V^{\prime}\right) \bmod \langle U\rangle$, hence the induced deformation along $\sigma^{\prime}$ is equiadapted to $\eta$ if and only if $L\left(1, V^{\prime}\right)=c\left(V^{\prime}+a\right)^{m}$. This proves the equivalence of conditions (i) and (ii). The uniqueness follows by comparing coefficients.

For a given $P \rightarrow R=P /\langle f\rangle$ and any $k \geqslant 0$, one has the curve diagram $\mathbf{R}^{(k)}$ consisting of all infinitely near points $P^{\prime}$ of $P$ on $R$ which lie in a neighbourhood of order $\nu \leqslant k$ and such that either $R^{\prime}$ is singular at $P^{\prime}$ or $P^{\prime}$ is not consecutive to a point $P^{\prime \prime}$ such that $R^{\prime \prime}$ is smooth. Note that $\mathbf{R}^{(0)}$ consists of the data $(P, R)$ and that $\mathbf{R}^{(1)}$ is nothing but the blow-up diagram $\mathscr{R}_{P, f}$ of $P \rightarrow P /\langle f\rangle$. Denote by $h$ the maximum $k$ such that $\mathbf{R}^{(k-1)} \neq \mathbf{R}^{(k)}$. Set $\mathbf{R}=\mathbf{R}^{(h)}$. 


\section{EQUISINGULAR DEFORMATIONS IN ARBITRARY CHARACTERISTIC}

The category (respectively, functor of isomorphism classes) of ep-deformations of $\mathbf{R}^{(k)}$ is denoted by $\mathcal{D} e f_{\mathbf{R}^{(k)}}^{\text {ep }}$ (respectively, $\underline{\mathcal{D}}_{\mathbf{R}^{(k)}}^{\text {ep }}$ ). For $k=h$, we simply write $\mathcal{D} e f_{\mathbf{R}}^{\text {ep }}$ (respectively, $\underline{\mathcal{D} e f} f_{\mathbf{R}}^{\text {ep }}$ ) for the category $\mathcal{D} e f_{\mathbf{R}^{(h)}}^{\mathrm{ep}}$ (respectively, the functor $\underline{\mathcal{D}}_{\mathbf{R}^{(h)}}^{\mathrm{ep}}$ ).

The following lemma shows that the deformations in $\mathcal{D} e f_{\mathbf{R}}^{\text {ep }}$ can also be considered as deformations of the parametrization.

LEMma 7.10. There is a natural functor $\mathcal{D} e f_{\mathbf{R}}^{\mathrm{ep}} \rightarrow \mathcal{D} e f_{\bar{R} \leftarrow P}^{\mathrm{sec}}$ which identifies $\mathcal{D}$ ef $\mathbf{R}_{\mathbf{R}}^{\mathrm{ep}}$ with a full subcategory of $\mathcal{D} e f \frac{\mathrm{sec}}{R} \leftarrow P$.

Proof. Each $\xi \in \mathcal{D}$ ef $f_{\mathbf{R}}^{\text {ep }}$ is equipped with a section of $A \rightarrow Q_{A}$ for each $Q$ in $\mathbf{R}$, in particular, with sections $\sigma_{i}: Q_{i, A} \rightarrow A$ for the maximal points $Q_{1}, \ldots, Q_{r}$ in $\mathbf{R}$, that is, those essential points where $R_{Q}$ is smooth. After relabelling, $Q_{1}, \ldots, Q_{r}$ correspond one to one to the branches $R_{1}, \ldots, R_{r}$ of $R$, in the sense that $Q_{i}$ is on $R_{i}$ and not on $R_{j}$ for $j \neq i$. Now, the deformation $Q_{i, A} \rightarrow R_{Q_{i}, A}=\bar{R}_{Q_{i}, A}$ given by $\xi$ becomes a deformation $Q_{i, A} \rightarrow \bar{R}_{i, A}$ of $\bar{R}_{i}$, and $\sigma_{i}$ induces a section $\bar{\sigma}_{i}: \bar{R}_{i, A} \rightarrow A$ as it lies inside $R_{i, A}$. The $\bar{\sigma}_{i}$ all together give rise to $\bar{\sigma}: \bar{R}_{A} \rightarrow A$ and the statement of the lemma follows from this fact.

\subsection{Equipolygonal deformations of the parametrization}

Next, we consider equipolygonal deformations in the parametric case. For this, choose an infinitely near point $Q$ of $P$, a parametrization $\varphi_{Q}: Q \rightarrow \bar{R}_{Q}$ of $R_{Q}$, and adapted elements $u, v$ to $H_{Q}$. Up to permutation we may assume that $i\left(E_{Q}, Q /\langle u\rangle\right) \geqslant e, i\left(D_{Q}, Q /\langle v\rangle\right) \geqslant d$ if $E_{Q}, D_{Q}$ exist.

For $i \in \Lambda_{Q}$, denote by $m_{e, i}=\operatorname{ord} \varphi_{Q, i}(u)$ (respectively, $m_{d, i}=\operatorname{ord} \varphi_{Q, i}(v)$ ) if $E_{Q}$ (respectively, $D_{Q}$ ) exists, and $m_{e, i}=m_{i}$ (respectively, $m_{d, i}=m_{i}$ ) otherwise (see Definition 2.1 for notations). The following objects depend only on $Q$ and $R_{Q} \rightarrow \bar{R}_{Q}$ :

(i) the submodule $I_{\bar{R}_{Q} \leftarrow R_{Q}}^{\mathrm{ep}}=\bigoplus_{i \in \Lambda_{Q}}\left(\overline{\mathfrak{m}}_{i}^{m_{e, i}} \oplus \overline{\mathfrak{m}}_{i}^{m_{d, i}}\right)$ of $\bar{R}_{Q} \oplus \bar{R}_{Q}$;

(ii) the adapted Jacobian module given by

$$
J_{Q, \bar{R} \leftarrow R}=\overline{\mathfrak{m}}(\dot{u}, \dot{v})+\left(\left\langle u, v^{d}\right\rangle \oplus\left\langle u^{e}, v\right\rangle\right) ;
$$

(iii) the finite-dimensional $K$-vector space $T_{Q \bar{R} \leftarrow R}^{\mathrm{ep}}=I_{\bar{R}_{Q} \leftarrow R_{Q}}^{\mathrm{ep}} / J_{Q, \bar{R} \leftarrow R}$.

Definition 7.11. Let $\eta=\left(\phi_{A}, \bar{\sigma}, \sigma\right) \in \mathcal{D} e f \frac{\mathrm{sec}}{\bar{H}_{Q} \leftarrow Q}(A)$ and $\zeta \in \mathcal{D} e f \frac{\sec }{R_{Q} \leftarrow Q}(A)$. Then we say that $\zeta$ is adapted to $\eta$ if $\zeta=\left(\varphi_{A}, \bar{\sigma}^{\prime}, \sigma\right)$ fits into a commutative diagram

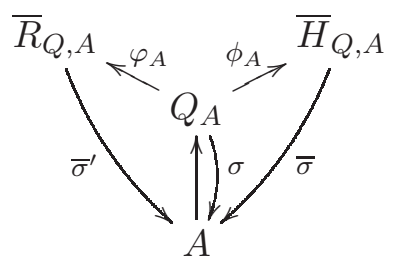

that is, $\zeta$ and $\eta$ are deformations with the same section $\sigma$.

Choose generic adapted elements $u, v \in Q$ and $U, V \in I_{\sigma} \subset Q_{A}$ adapted to $\eta$ such that $u \equiv U$ $\bmod \mathfrak{m}_{A}$ and $v \equiv V \bmod \mathfrak{m}_{A}$. Assume that $U$ (respectively, $V$ ) corresponds to $E_{Q, A}$ (respectively, $D_{Q, A}$ ) if $E_{Q}$ (respectively, $D_{Q}$ ) exists. Then the adapted deformation $\zeta$ is called equiadapted to $E_{Q, A}$ (respectively, $D_{Q, A}$ ) if for any $i \in \Lambda_{Q}$ one has $\operatorname{ord} \varphi_{Q, A, i}(U)=\operatorname{ord} \varphi_{Q, i}(u)$ (respectively, ord $\left.\varphi_{Q, A, i}(V)=\operatorname{ord} \varphi_{Q, i}(v)\right)$.

The adapted deformation $\zeta$ is said to be equipolygonal if it is equimultiple in the sense of Remark 2.7(2) and if it is equiadapted to $E_{Q, A}$ and to $D_{Q, A}$ whenever $E_{Q}$ and $D_{Q}$ exist. Note that if $\zeta$ is equipolygonal, then its image in $\mathcal{D} e f_{R_{Q}}^{\mathrm{sec}}(A)$ is equipolygonal in the sense of Definition 7.2. The converse is not true as Remark 2.7(3) shows. 


\section{A. Campillo, G.-M. Greuel and C. Lossen}

The above definitions of equiadapted and equipolygonal deformations do not depend on the choice of the (generic) adapted elements $u, v, U, V$.

Denote by $\mathcal{D} e \bar{R}_{\bar{R}_{Q} \leftarrow R_{Q}}^{\text {ep }}$ (respectively, $\underline{\mathcal{D} e f} \bar{R}_{Q} \overline{\mathrm{e}}_{Q} \leftarrow R_{Q}$ ) the category of equipolygonal deformations (respectively, of adapted isomorphism classes of equipolygonal deformations) of the parametrization. The vector space $T_{\bar{R}_{Q} \leftarrow R_{Q}}^{\mathrm{ep}}$ can be considered as the tangent space to $\underline{\mathcal{D} e f} \bar{R}_{Q \leftarrow R_{Q}}$. For each $\zeta \in$ $\mathcal{D} e f_{\bar{R}_{Q} \leftarrow R_{Q}}^{\mathrm{ep}}(A)$, there is a well-defined linear (Kodaira-Spencer) map $\psi_{\zeta}: T_{A} \rightarrow T_{Q, \bar{R} \leftarrow R}^{\mathrm{ep}}$ similarly to the non-parametric case. The deformation $\zeta$ is said to be ep-versal if its Kodaira-Spencer map $\psi_{\zeta}$ is surjective. If $\psi_{\zeta}$ is an isomorphism, then we call $\zeta$ equipolygonal semiuniversal or ep-semiuniversal.

Proposition 7.12. Let $\left(a_{1}, b_{1}\right), \ldots,\left(a_{s}, b_{s}\right) \in I_{\bar{R}_{Q} \leftarrow R_{Q}}^{\mathrm{ep}}$ represent a basis (respectively, a system of generators) of $T_{Q, \bar{R} \leftarrow R}^{\mathrm{ep}}$. Then

$$
U=u+\sum_{i=1}^{s} T_{i} a_{i}, \quad V=v+\sum_{i=1}^{s} T_{i} b_{i}
$$

defines an ep-semiuniversal (respectively, ep-versal) deformation of $Q \rightarrow \bar{R}$.

The proof is trivial, cf. Proposition 7.3. Moreover, Remark 7.4 applies mutatis mutandis.

In particular, each ep-versal deformation of $\bar{R}_{Q} \leftarrow R_{Q}$ has a smooth basis.

Definition 7.13. A parametric curve diagram $(\mathscr{C}, \overline{\mathscr{G}})$ is a curve diagram as in Definition 7.5 for which the curve $Q \rightarrow Q /\langle g\rangle$ at each point $Q$ is given by a specified parametrization $\varphi_{Q}: Q \rightarrow \bar{R}_{Q}$, that is, a diagram consisting of $\mathscr{C}$ and the list of parametrizations $\left(\varphi_{Q}\right)_{Q \in \mathscr{C}}$.

An equipolygonal deformation of a parametric curve diagram $(\mathscr{C}, \overline{\mathscr{G}})$ is a list of equipolygonal deformations of $Q_{A} \rightarrow \bar{R}_{Q, A}, Q \in \mathscr{C},\left(\varphi_{Q}: Q \rightarrow \bar{R}_{Q}\right) \in \overline{\mathscr{G}}$, adapted to the given deformations of $E_{Q}, D_{Q}$ such that the obvious analogues to Definition 7.7 (1)-(3) are satisfied.

For a parametric curve diagram $(\mathscr{C}, \overline{\mathscr{G}})$ one defines

$$
\mathbf{T}^{\mathrm{ep}}=\mathbf{T}_{(\mathscr{C}, \overline{\mathscr{G}})}^{\mathrm{ep}}=\bigoplus_{Q \in \mathscr{C}} T_{Q, \bar{R} \leftarrow R}^{\mathrm{ep}} .
$$

An ep-deformation of $(\mathscr{C}, \overline{\mathscr{G}})$ is said to be ep-versal if the obvious Kodaira-Spencer map $T_{A} \rightarrow \mathbf{T}^{\mathrm{ep}}$ is surjective.

The parametric analogues to Proposition 7.8 and Lemmas 7.9 and 7.10 also hold and they are rather trivial as shown below.

First, the parametric multicurve of tangential components of a given $\varphi: Q \rightarrow \bar{R}_{Q}$ is given by $\mathscr{C}=(Q, \ldots, Q)(s$ entries, where $s$ is the number of tangential components) and the lists $\overline{\mathscr{G}}$ of the parametrizations $\varphi_{j}$ of the curves given by the branches which share one of the tangents.

Proposition 7.14. Let $\zeta \in \mathcal{D}$ ef $\overline{\bar{R}}_{Q \leftarrow R_{Q}}^{\mathrm{ep}}$. Denote by $\zeta_{(\mathscr{C}, \overline{\mathscr{G}})}$ the deformation of the parametric multicurve of tangential components obtained by distributing the deformations of the parametrizations of the branches of $\bar{R}_{Q}$ according to their tangents. Then one has:

(1) $\zeta_{(\mathscr{C}, \overline{\mathscr{G}})}$ is an equipolygonal deformation of $(\mathscr{C}, \overline{\mathscr{G}})$;

(2) if $\zeta$ is ep-versal, then $\zeta_{(\mathscr{C}, \bar{G})}$ is ep-versal too.

Proof. Part (1) follows from the definitions and part (2) from the fact that the map $T_{Q, \bar{R} \leftarrow R}^{\mathrm{ep}} \rightarrow$ $\mathbf{T}_{(\mathscr{C}, \overline{\mathscr{G}})}^{\mathrm{ep}}$ is obviously surjective. 


\section{EQUISINGULAR DEFORMATIONS IN ARBITRARY CHARACTERISTIC}

Now, assume that $R_{Q}$ has only one tangential component and denote by $\widetilde{\zeta}$ the transform at $Q_{A}^{\prime}$ of $\zeta \in \mathcal{D} e f_{\bar{R}_{Q} \leftarrow R_{Q}}^{\text {ep }}(A)$ under the blowing up of the section $\sigma$ given by $\zeta$. One has either $m_{i}=m_{e, i} \leqslant m_{d, i}$ for all $i \in \Lambda_{Q}$ or $m_{i}=m_{d, i} \leqslant m_{e, i}$ for all $i \in \Lambda_{Q}$. Without loss of generality, we assume the first case. Then, since the leading term of $\varphi_{Q, A, i}(U)$ is a unit, one has that the leading term $a_{i}$ of $\varphi_{Q, A, i}(V) / \varphi_{Q, A, i}(U)$ is a well-defined element of $A$. Then the following lemma is again trivial.

Lemma 7.15. Let $R_{Q}$ be unitangential. Then the deformation $\widetilde{\zeta}$ is equiadapted to $E_{Q^{\prime}, A}$ along the section $\sigma^{\prime}$ if and only if one has $a_{i}=a_{i^{\prime}}$ for every couple $i, i^{\prime} \in \Lambda_{Q}$. In that case, if $a=a_{i}, i \in \Lambda_{Q}$, is the common value, then the section $\sigma^{\prime}$ is given by $\langle u, v / u-a\rangle$.

Finally, for a given $P \rightarrow \bar{R}$ and $k \geqslant 0$, denote by $\overline{\mathbf{R}}^{(k)}$ the parametric curve diagram consisting of the points $Q$ of $\mathbf{R}^{(k)}$ together with the parametrizations $Q \rightarrow \bar{R}_{Q}$. The category (respectively, functor) of ep-deformations of $\overline{\mathbf{R}}^{(k)}$ will be denoted by $\mathcal{D} e f \overline{\mathbf{R}}^{\text {ep }}(k)$ (respectively, $\left.\frac{\mathcal{D} e f}{{ }^{e p}} \overline{\mathbf{R}}^{(k)}\right)$. For $k=h$, we simply write $\mathcal{D} e f_{\overline{\mathbf{R}}}^{\mathrm{ep}}$ (respectively, $\underline{\mathcal{D} e f} \frac{\mathrm{ep}}{\overline{\mathbf{R}}}$ ) for $\mathcal{D} e f_{\overline{\mathbf{R}}^{(h)}}^{\mathrm{ep}}$ (respectively, $\underline{\mathcal{D} e f} \overline{\mathbf{R}}^{\mathrm{ep}}{ }^{(h)}$ ). Note that one has natural maps $\mathcal{D} e f_{\overline{\mathbf{R}}^{(k)}}^{\mathrm{ep}} \rightarrow \mathcal{D} f_{R^{(k)}}^{\mathrm{ep}}$.

LEmma 7.16. There is a natural functor $\mathcal{D} e f \frac{\mathrm{ep}}{\overline{\mathbf{R}}} \rightarrow \mathcal{D} e f \frac{\mathrm{sec}}{\bar{R} \leftarrow P}$ which identifies $\mathcal{D}$ ef $\frac{\mathrm{ep}}{\mathbf{R}}$ with a full subcategory of $\operatorname{Def} \frac{\mathrm{sec}}{R} \leftarrow P^{\text {. }}$

Proof. The composite of the functor in Lemma 7.10 with the natural functor $\mathcal{D}$ ef $\overline{\mathbf{R}} \rightarrow \mathcal{D}$ ef $f_{\mathbf{R}}^{\mathrm{ep}}$ corresponding to $k=h$ yields the required functor in the lemma.

Now, we come to the main result of this section which shows that ep-deformations of $\mathbf{R}$, epdeformations of $\overline{\mathbf{R}}$ and equisingular deformations of the parametrization are essentially the same objects.

Theorem 7.17. For a given parametrization $P \rightarrow \bar{R}$, the categories $\mathcal{D}$ ef $\frac{\mathrm{ep}}{\mathbf{R}}$, $\mathcal{D} e f_{\mathbf{R}}^{\mathrm{ep}}$, and $\mathcal{D}$ ef $\frac{\mathrm{es}}{\bar{R}} P$ (respectively, the functors $\underline{\mathcal{D} e f}_{\mathbf{R}}^{\mathrm{ep}}, \underline{\mathcal{D}}_{\mathbf{R}}^{\mathrm{ep}}$, and $\underline{\mathcal{D} e f} \bar{R}_{\leftarrow P}^{\mathrm{es}}$ ) are pairwise equivalent (respectively, pairwise isomorphic).

Proof. By Lemmas 7.10 and 7.16 there are natural maps $\mathcal{D} e f_{\overline{\mathbf{R}}}^{\mathrm{ep}} \rightarrow \mathcal{D} e f_{\mathbf{R}}^{\mathrm{ep}} \rightarrow \mathcal{D} e f \frac{\mathrm{sec}}{\bar{R} \leftarrow P}$ which identify $\mathcal{D} e f_{\overline{\mathbf{R}}}^{\mathrm{ep}}$ and $\mathcal{D} e f_{\mathbf{R}}^{\mathrm{ep}}$ with respective full subcategories of $\mathcal{D} e f_{\bar{R} \leftarrow P}^{\mathrm{sec}}$. We claim that both subcategories are equal and, in fact, also equal to the subcategory $\mathcal{D} e f_{\bar{R} \leftarrow P}^{\text {es }}$ of $\mathcal{D} e f \frac{\text { sec }}{\bar{R} \leftarrow P}$. The theorem follows from the claim.

To prove the claim, it is enough to show two statements:

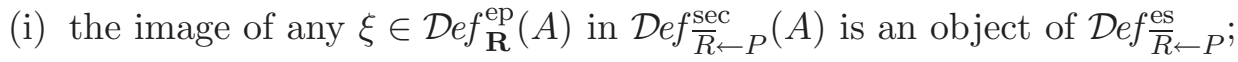

(ii) for any $\zeta \in \mathcal{D} e f \frac{\text { es }}{R \leftarrow P}(A)$ there exists $\xi \in \mathcal{D} e f \frac{\text { ep }}{\mathbf{R}}(A)$ having $\zeta$ as image in $\operatorname{Def}_{R \leftarrow P}^{\text {sec }}(A)$.

First, for $\xi \in \mathcal{D e f}_{\mathbf{R}}^{\mathrm{ep}}(A)$ consider its image $\zeta \in \mathcal{D e f}_{\bar{R} \leftarrow P}^{\mathrm{sec}}(A)$ and, for each $Q$, the deformation $\xi_{Q} \in \mathcal{D} e f \bar{R}_{Q \leftarrow Q}^{\sec }(A)$ given by the data $(\xi, \zeta)$. To check statement (i) one needs to show that $\xi_{Q}$ is equiadapted to $E_{Q, A}, D_{Q, A}$ (if $E_{Q}, D_{Q}$ exist) and, moreover, an equimultiple deformation of the parametrization. We will show this by recurrence on the integer $h$. For $h=0$, it is trivial. Now, take $Q$ and $U, V$ adapted to $Q$. By recurrence, assume that $\xi_{Q^{\prime}}$ is equiadapted to $E_{Q^{\prime}, A}, D_{Q^{\prime}, A}$ (if they exist) and an equimultiple deformation of the parametrization for all $Q^{\prime}$ in the first neigbourhood of $Q$. For fixed $Q^{\prime}$, assume (without loss of generality) that $u=U \bmod \mathfrak{m}_{A}$ is transversal to $R_{Q, i}$ for all $i \in \Lambda_{Q^{\prime}}$. If $Q^{\prime}$ is satellite, then one component of $H_{Q}$ will be tangent to all $R_{Q, i}$ with $i \in \Lambda_{Q^{\prime}}$, and $V$ is nothing but an equation for that component. If $\varphi_{A}$ denotes the deformation of the parametrization given by $\zeta$ then, since $U$ becomes an equation for $E_{Q^{\prime}, A}$, one has ord $\varphi_{Q^{\prime}, A, i}(U)=$ $\operatorname{ord} \varphi_{Q^{\prime}, i}(u)$ for $i \in \Lambda_{Q^{\prime}}$. Since $V=V^{\prime} U+a U$ with $a \in A$ and $U, V$ generators of $I_{\sigma^{\prime}}$, one has $\operatorname{ord} \varphi_{Q, A, i}(V) \geqslant \operatorname{ord} \varphi_{Q, A, i}(U)=\operatorname{ord} \varphi_{A, i}(u)$. The parametric adaptedness to the deformation of the 


\section{A. Campillo, G.-M. Greuel and C. Lossen}

nontangent components of branches with $i \in \Lambda_{Q^{\prime}}$, as well as the parametric equimultiplicity for $\xi_{Q}$ with respect to those branches, follows from the above fact. On the other hand, if $Q^{\prime}$ is satellite, then $I_{\sigma^{\prime}}=\left\langle U, V^{\prime}\right\rangle$ with $V^{\prime}=V / U$. By the recurrence hypothesis one has ord $\varphi_{Q^{\prime}, A, i}(V)=\operatorname{ord} \varphi_{Q^{\prime}, i}\left(v^{\prime}\right)$, hence ord $\varphi_{Q, A, i}(V)=\operatorname{ord} \varphi_{Q, i}(v)$ which shows that the parametric equiadaptness condition also holds for the deformation of the tangent component of $H_{Q}$ for $\xi_{Q}$ with respect to the branches with $i \in \Lambda_{Q^{\prime}}$. This shows statement (i).

Second, take $\zeta \in \mathcal{D} e f \frac{\mathrm{es}}{\bar{R} \leftarrow P}(A)$. Then $\zeta$ induces deformations $\zeta_{Q} \in \mathcal{D}$ ef $\frac{\sec }{\bar{R}_{Q} \leftarrow Q}(A)$ which are parametric equimultiple. To check statement (ii) one needs to prove that they are also parametric equiadapted to $E_{Q, A}, D_{Q, A}$. From this one sees that $\zeta_{Q}$ gives rise to a list of ep-deformations of the

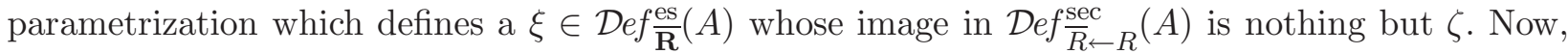
we prove this by recurrence on the order of the infinitesimal neighbourhood $\nu$ of $Q$. For $\nu=0$, this is trivial. Assume that it is true for $Q$ and take $Q^{\prime}$ in the first neighbourhood of $Q$ and choose adapted $U, V$ at $Q$ such that the equations of the components of $H_{Q, A}$ are either $U$ or $V$. Also assume $u=U\left(\bmod \mathfrak{m}_{A}\right)$ is transversal to $R_{Q, i}$ for $i \in \Lambda_{Q}$ so that one has ord $\varphi_{Q, A, i}(U)=\operatorname{ord} \varphi_{Q, i}(u)$ ( $\varphi_{A}$ being the data given by $\zeta$ ). Since $U$ is an equation for $E_{Q^{\prime}, A}$ it follows that $\zeta_{Q^{\prime}}$ is equiadapted to $E_{Q^{\prime}, A}$. If $Q^{\prime}$ is satellite, then $V^{\prime}=V / U$ is an equation for $D_{Q^{\prime}, A}$ and $V$ is an equation for either $D_{Q^{\prime}, A}$ or $D_{Q, A}$, By recurrence one has ord $\varphi_{Q, A, i}(V)=\operatorname{ord} \varphi_{Q, i}(v)$, hence one deduces ord $\varphi_{Q, A, i}\left(V^{\prime}\right)=\operatorname{ord} \varphi_{Q^{\prime}, i}\left(v^{\prime}\right)$, where $v=V\left(\bmod , \mathfrak{m}_{A}\right), v^{\prime}=V^{\prime}\left(\bmod \mathfrak{m}_{A}\right)$. This proves the statement (ii) and completes the proof of the theorem.

\section{Proofs for weakly equisingular strata}

To prove Theorem 6.2, we need some preparations by means of some constructions, properties, and notation.

Let $Q$ be an infinitely near point of $P$ on $R$, and let $u, v$ be generic adapted coordinates (to $\left.H_{Q}\right)$. Let $g \in Q=K[[u, v]]$ define a non-exceptional curve, and let $\eta=\eta_{Q} \in \mathcal{D} e f_{Q /\langle g\rangle}^{\mathrm{ep}}(C)$ be defined by $G(U, V) \in C[[U, V]]$ and $I_{\sigma}=\langle U, V\rangle$, where $U, V$ are adapted, $u \equiv U \bmod \mathfrak{m}_{C}, v \equiv V$ $\bmod \mathfrak{m}_{C}$. Then $G-g \in I_{R_{Q}}^{\text {ep }} C[[U, V]]$ and, by Proposition 7.8, we can assume that $G$ decomposes as $G=G_{1} \cdots G_{s}$, where $G_{j}$ defines an equipolygonal deformation of the tangential component $g_{j}$ of $g, j=1, \ldots, s$. Up to relabelling the components, we may assume that $g_{1}, \ldots, g_{s^{\prime}}$ are not tangential to $H_{Q}$, and that $g_{s^{\prime}+1}, \ldots, g_{s}$ are tangential to $H_{Q}$. Note that $0 \leqslant s-s^{\prime} \leqslant 2$. As above, we assume additionally that $u$ is transversal to $g_{j}, j=1, \ldots, s^{\prime}$.

We introduce new indeterminates $\mathbf{W}=\left(W_{1}, \ldots, W_{s^{\prime}}\right)$ and consider the following ideals $I_{j} \subset$ $C[[\mathbf{W}]], j=1, \ldots, s$ : if $j>s^{\prime}$, set $I_{j}:=\langle 0\rangle$. If $j \leqslant s^{\prime}$, the $m_{j}$-jet of $G_{j}\left(m_{j}\right.$ the multiplicity of $\left.G_{j}\right)$ reads

$$
L_{j}=c_{j}\left(V^{m_{j}}+c_{1, j} V^{m_{j}-1} U+\cdots+c_{m_{j}, j} U^{m_{j}}\right), \quad c_{j} \in C^{*}, c_{i j} \in C .
$$

Write $m_{j}=q_{j} m_{j}^{\prime}$, such that $q_{j}$ is the largest power of the characteristic $p$ dividing $m_{j}$ (if $p=0$, we set $q_{j}=1$ ). Then we set $I_{j}$ to be the ideal generated by the following elements:

(i) $c_{i, j}$, for $i$ not a multiple of $q_{j}$;

(ii) $c_{\ell q_{j}, j}-\left(\begin{array}{c}m_{j}^{\prime} \\ j\end{array}\right) c_{q_{j}, j}^{\ell}$, for $\ell=2, \ldots, m_{j}^{\prime}$;

(iii) $W_{j}^{q_{j}}+\gamma_{j}^{q_{j}}-\left(1 / m_{j}^{\prime}\right) c_{q_{j}, j}$, where $\gamma_{j} \in K, \gamma_{j}^{q_{j}} \equiv\left(1 / m_{j}^{\prime}\right) c_{q_{j}, j} \bmod \mathfrak{m}_{C}$,

(iv) the coefficients $d_{i, k}^{(j)}$ of $U^{i} V^{\prime k},(i, k) \notin N_{Q^{\prime}, R}$, in the transformed polynomial $G_{j}^{\prime}=U^{-m}$ $G\left(U, U V^{\prime}-U W_{j}-U \gamma_{j}\right), m=\sum_{j=1}^{s} m_{j}$.

Set $C^{\prime}=C[[\mathbf{W}]] /\left(I_{1}+\cdots+I_{s}\right)$, and let $\gamma: C \rightarrow C^{\prime}$ be the natural morphism. Then the induced deformation $\gamma \eta$ is an ep-deformation which can be extended to an ep-deformation $\eta^{(1)} \in \mathcal{D} e f_{\mathscr{R}_{Q, g}}^{\text {ep }}\left(C^{\prime}\right)$ 


\section{EQUISINGULAR DEFORMATIONS IN ARBITRARY CHARACTERISTIC}

of the blow-up diagram $\mathscr{R}_{Q, g}$. In fact, this follows by construction: let $Q^{\prime}$ be the point in the first neighbourhood of $Q$ corresponding to the tangent direction of $g_{j}$. Then the vanishing of the polynomials in elements (i)-(iii) guarantees that $L_{j}$ is a pure power, $L_{j}=c_{j}\left(V+U \gamma_{j}+U W_{j}\right)^{m_{j}}$, and the corresponding strict transform at $Q^{\prime}$ reads $c_{j}\left(V^{\prime}+W_{j}\right)^{m_{j}}$. Thus, we may choose as section $\sigma^{\prime}$ through $Q^{\prime}$ the section given by $\left\langle U, V^{\prime}+W_{j}\right\rangle$ if $j \leqslant s^{\prime}$, respectively the one given by $\langle U, V / U\rangle$ if $j>s^{\prime}$ and $g_{j}$ is tangent to $v$, respectively $\langle U / V, V\rangle$ if $j>s^{\prime}$ and $g_{j}$ is tangent to $u$. The vanishing of the coefficients in element (iv) gives that the deformation is, indeed, an ep-deformation.

Remark 8.1. The above construction has the following universal property. For any morphism $\chi$ : $C \rightarrow A$ and any extension of the induced deformation $\chi(\eta)$ to an ep-deformation $\xi$ of $\mathscr{R}_{Q, g}$ over $A$, there exists a unique map $\chi^{\prime}: C^{\prime} \rightarrow A$ such that $\chi^{\prime} \circ \gamma=\chi$ and $\xi=\chi^{\prime}\left(\eta^{(1)}\right)$. In fact, it follows from the property that the elements in $A$ analogous to the generators (i)-(iv) of the ideals $I_{j} \subset C$ are equal to zero, if one takes for $W_{j}$ the value $a$ in Lemma 7.9(ii) which corresponds to the section of $\xi$ at $Q_{j, A}^{\prime}$. For $j>s^{\prime}, Q_{j}^{\prime}$ is satellite and, therefore, the section is given by the intersection of $E_{Q_{j, A}^{\prime}}$ and $D_{Q_{j, A}^{\prime}}$, so by Definition $7.7(2)$ and (3) one has $a=0$. This shows that for $j>s^{\prime}$ no variables $W_{j}$ are needed.

Denote by $\mathscr{R}_{Q, g}^{\prime}$ the multicurve obtained by deleting $Q$ and $R_{Q}$ from the blow-up diagram $\mathscr{R}_{Q, g}$. Denote by $\eta^{\prime}$ the ep-deformation of $\mathscr{R}_{Q, g}^{\prime}$ obtained by deleting the deformation associated to $Q$ (that is, $\gamma \eta$ ) from the list of deformations of $\eta^{(1)}$.

The following proposition is the key step for proving Theorem 6.2.

Proposition 8.2. With the above assumptions and notation, if $\eta \in \mathcal{D e f}_{R_{Q}}^{\mathrm{ep}}(B)$ is an ep-versal deformation of $R_{Q}$ with smooth base $B$, then the deformation $\eta^{\prime} \in \mathcal{D} e f_{\mathscr{R}_{Q, g}^{\prime}}^{\mathrm{ep}}\left(B^{\prime}\right)$ is ep-versal and its base $B^{\prime}$ is also smooth.

Proof. First, assume that $s=1$ and set $Q_{1}^{\prime}=Q^{\prime}, g_{1}^{\prime}=g^{\prime}, G_{1}^{\prime}=G^{\prime}$. One has two possibilities: (i) $s^{\prime}=0$; (ii) $s^{\prime}=1$.

Case (i): Take adapted $U, V$ with $v \equiv V\left(\bmod \mathfrak{m}_{B}\right)$ tangent to $g$. Then the formal blow-up $Q \rightarrow Q^{\prime}$ (respectively, $Q_{B} \rightarrow Q_{B}^{\prime}$ ) is given by $u=u, v=v^{\prime} u$ (respectively, $U=U, V=V^{\prime} U$ ). The blowingup transformation $\Xi$ maps $u^{i} v^{j}$ to $u^{i+j-m} v^{\prime j}$. Moreover, one has $\Xi\left(I_{R_{Q}}^{\mathrm{ep}}\right) \subset I_{R_{Q^{\prime}}}^{\mathrm{ep}}, \Xi\left(J_{Q, R}\right) \subset J_{Q^{\prime}, R}$ and, hence, $\Xi$ induces a linear map $\Xi: T_{Q, R}^{\mathrm{ep}} \rightarrow T_{Q^{\prime}, R}^{\mathrm{ep}}$. Since $g^{\prime} \in J_{Q^{\prime}, R}$ and since the monomial $v^{\prime m}$ occurs in the support of $g^{\prime}$, the vector space $T_{Q^{\prime}, R}^{\mathrm{ep}}$ can be generated by monomials of the image of $\Xi$ (for instance, by the basic monomials with respect to a monomial ordering for which $v^{\prime m}$ is the leading term of $\left.g^{\prime}\right)$. It follows that $\bar{\Xi}$ is surjective.

On the other hand, one has $\psi_{\eta^{\prime}}=\Xi \circ \psi_{\eta}, \psi_{\eta}, \psi_{\eta^{\prime}}$ being the Kodaira-Spencer maps for $\eta$ and $\eta^{\prime}$, respectively. Since $\bar{\Xi}$ is surjective, one concludes that $\eta^{\prime}$ is ep-versal and defined over the same base $B$ as $\eta$. So, $B^{\prime}=B$ is smooth.

Case (ii): Take adapted $U, V$ with $u \equiv U\left(\bmod \mathfrak{m}_{B}\right)$ transversal to $g$. Since $Q^{\prime}$ is free and $U$ is an equation for $E_{Q^{\prime}, B}$ at $Q_{B}^{\prime}$, in place of $U, V$ we will consider the couple of generators $U, Z$ of $I_{\sigma}$ where $Z=V+\lambda U$ and $\lambda \in K$ is such that $z \equiv Z\left(\bmod \mathfrak{m}_{B}\right)$ is tangent to $g$. If $b=\lambda+a$, one has $L=c(V-a U)^{m}$ if and only if $L=c(Z-b U)^{m}$ and, in that case, $I_{\sigma^{\prime}}=\left\langle U, V^{\prime}-a\right\rangle=\left\langle U, Z^{\prime}-b\right\rangle$ with $b \in \mathfrak{m}_{B}$. Moreover, $L$ needs not to be a pure power. Condition (ii) from the beginning of this section for (the construction of) $B^{\prime}$ guarantees that $L$ is a pure power of the above type for the induced deformation on $B^{\prime}$.

With the choice of $U, Z$, we set $\mathcal{P}_{0}=\left\{Q, Q^{\prime}\right\}, e_{0}=1, d_{0}=2$. This is an auxiliar notation (for the proof) which emphasizes that $u, z$ can be seen as adapted parameters to $\mathcal{P}_{0}$ as both $Q$ and $Q^{\prime}$ are on the curves $R_{Q}$ and $Q /\langle z\rangle$. Other couples $u, z_{0}$ such that $Q, Q^{\prime}$ are the only points in common 


\section{A. Campillo, G.-M. Greuel and C. Lossen}

on $R_{Q}$ and $Q /\langle z\rangle$ can be thought as generic for $\mathcal{P}_{0}$. The Newton polygon $N_{0}$ of $g$ with respect to generic couples for $\mathcal{P}_{0}$ is constant. Thus, one has the associated objects as follows:

(1) the Newton polygon $N_{0}$;

(2) the ideal $I_{Q, 0}^{\mathrm{ep}}$ generated by the monomials with exponents in $N_{0}+\mathbb{Z}_{\geqslant 0}^{2}$;

(3) $J_{Q, 0} \subset I_{Q, 0}^{\mathrm{ep}}$ the ideal generated by $g, u(\partial g / \partial u), u^{2}(\partial g / \partial z), z(\partial g / \partial u), z(\partial g / \partial z)$;

(4) the vector space $T_{Q, 0}^{\mathrm{ep}}=I_{Q, 0}^{\mathrm{ep}} / J_{Q, 0}$.

Note that one has $J_{Q, 0} \subset J_{Q, R}$ and $J_{Q, R}=J_{Q, 0}+(u(\partial g / \partial z)) K$ (but not necessarily $u(\partial g / \partial z) \notin$ $\left.J_{Q, 0}\right)$. We distinguish three subcases:

(ii) $1 \quad p \nmid m$ or $p=0$;

(ii) $2 p \mid m$ and $J_{Q, R} \subset I_{Q, 0}^{\mathrm{ep}}$;

(ii) $3 \quad p \mid m$ and $J_{Q, R} \not \subset I_{Q, 0}^{\mathrm{ep}}$.

Note that $J_{Q, 0}=J_{Q, R}$ is only possible in case (ii) $)_{2}$.

Fix an appropriate monomial ordering (for instance, the weighted degree reverse lexicographic ordering with respect to the steepest segment of the Newton polygon $\left.N_{0}\right)$. Let $\left\{g_{1}, \ldots, g_{\ell}\right\}$ be a standard basis for the ideal $J_{Q, 0}$ with respect to the fixed ordering. If $J_{Q, 0}=J_{Q, R}$, then, of course, $\left\{g_{1}, \ldots, g_{\ell}\right\}$ is also a standard basis for the ideal $J_{Q, R}$; if $J_{Q, 0} \subsetneq J_{Q, R}$, then $\left\{g_{1}, \ldots, g_{\ell}, u(\partial g / \partial z)\right\}$ is a standard basis for $J_{Q, R}$. Those monomials $n_{1}, \ldots, n_{d}$ in $I_{R_{Q}}^{\mathrm{ep}}$ which are not in the initial ideal of $J_{Q, R}$ induce a vector space basis of $T_{Q, R}^{\mathrm{ep}}$. Adding $u\left(\partial g / \partial z\right.$ ) (in case $J_{Q, 0} \subsetneq J_{Q, R}$ ), we get a vector space basis for $I_{R_{Q}}^{\mathrm{ep}} / J_{Q, 0}$.

The division theorem for standard bases now provides a unique way to write any element $h \in$ $I_{R_{Q}}^{\mathrm{ep}}$ as

$$
h=\sum_{i=1}^{d} t_{i} \cdot n_{i}+b \cdot u \frac{\partial g}{\partial z}+\sum_{j=1}^{\ell} c_{j}(u, z) \cdot g_{j}, \quad t_{i}, b \in K,
$$

where the term $b \cdot u(\partial g / \partial z)$ does not appear if $J_{Q, 0}=J_{Q, R}$, and where the $c_{j}(u, z)$ are power series with support in some regions $R_{j}$ determined by the division procedure. Such division also applies to $B[[u, z]]$. In fact, if $H \in I_{R_{Q}}^{\mathrm{ep}} B[[u, z]]$, then $H$ can be uniquely expressed in the form

$$
H=\sum_{i=1}^{d} t_{i} \cdot n_{i}+b \cdot u \frac{\partial g}{\partial z}+\sum_{j=1}^{\ell} C_{j}(u, z) \cdot g_{j}, \quad t_{i}, b \in \mathfrak{m}_{B},
$$

where $C_{j}(u, z) \in B[[u, z]]$ has support in the region $R_{j}$.

Now, consider the equation $G=g+H$ of the given ep-deformation $\eta$. One has $H \in I_{R_{Q}}^{\mathrm{ep}} B[[u, z]]$. By the above discussion, we have

$$
G=g+\sum_{i=1}^{d} t_{i} \cdot n_{i}+b \cdot u \frac{\partial g}{\partial z}+\sum_{j=1}^{\ell} C_{j}(u, z) \cdot g_{j}, \quad t_{i}, b \in \mathfrak{m}_{B},
$$

with $C_{j}(u, z) \in B[[x, y]]$ as above and $b$ only occurring if $J_{Q, 0} \subsetneq J_{Q, R}$. Since $\eta$ is ep-versal, there are derivations $\delta_{1}, \ldots, \delta_{d} \in T_{B}$ such that $\delta_{j}\left(t_{i}\right)=\delta_{j i}$. This follows from the fact that, for each $\delta \in T_{B}$, the Kodaira-Spencer map $\psi_{\eta}$ is surjective and given by $\psi_{\eta}(\delta)=\sum_{i=1}^{d} \delta\left(t_{i}\right) \cdot n_{i}$ modulo $J_{Q, R}$. Since $T_{B}=\left(\mathfrak{m}_{B} / \mathfrak{m}_{B}^{2}\right)^{*}$, it follows that $t_{1}, \ldots, t_{d}$ are part of a regular system of parameters for $B$.

Note also that, without loss of generality, we can assume that $g$ and $G$ can be chosen as Weierstraß polynomials in $z$ of degree $n$. 


\section{EQUISINGULAR DEFORMATIONS IN ARBITRARY CHARACTERISTIC}

Case (ii) $)_{1}$ : Here, the leading term of $u(\partial g / \partial z)$ is given by $m b u z^{m-1}$. Thus, $u z^{m-1}$ is not a basic monomial, while $u^{2} z^{m-2}, \ldots, u^{m-1} z, u^{m}$ are. Assume the latter are the basic monomials $n_{2}, \ldots, n_{m}$. Then $t_{2}, \ldots, t_{m}$ are part of a regular system of parameters, and the leading form of $G$ is given by

$$
L=z^{m}+m b u z^{m-1}+t_{2} u^{2} z^{m-2}+\cdots+t_{m} u^{m} .
$$

Conditions (i) and (ii) for $B^{\prime}$ impose that $L=(z-a u)^{m}$ for some $a \in B$, which is equivalent to $b=-a$ and $t_{i}-\left(\begin{array}{c}m \\ i\end{array}\right) b^{i}=0$ for $i=2, \ldots, m$. Since $b^{i} \in \mathfrak{m}_{B}^{2}$ for $i \geqslant 2$, these conditions are analytically independent. Thus, the quotient ring $\widetilde{B}$ of $B$ modulo the ideal generated by these $m-1$ smooth conditions is again regular. The induced ep-deformation on it has an equation $\widetilde{G}$ whose leading term is the pure power $(z+b u)^{m}$. Now, substituting $\widetilde{z}:=z+b u$ (which preserves adaptation to the data $\mathcal{P}_{0}$, hence, does not change the analytical description of the Kodaira-Spencer map), we get

$$
\begin{aligned}
\widetilde{G}(u, \widetilde{z}-b u)= & g(u, \widetilde{z}-b u)+t_{1} n_{1}(u, \widetilde{z}-b u)+\sum_{i=m}^{d} t_{i} n_{i}(u, \widetilde{z}-b u) \\
& +b u \frac{\partial g}{\partial z}(u, \widetilde{z}-b u)+\sum_{j=1}^{\ell} C_{j}(u, \widetilde{z}-b u) \cdot g_{j}(u, \widetilde{z}-b u) \\
= & g(u, \widetilde{z})+t_{1} n_{1}(u, \widetilde{z})+\sum_{i=m}^{d} t_{i} n_{i}(u, \widetilde{z})+\sum_{j=1}^{\ell} C_{j}(u, \widetilde{z}) g_{j}(u, \widetilde{z})+r(u, \widetilde{z}),
\end{aligned}
$$

where $C_{j}$ has coefficients in $\mathfrak{m}_{\widetilde{B}}$ and $r$ has coefficients in $\mathfrak{m}_{\widetilde{B}}^{2}$. Let $r_{i}$ be the coefficient of $r$ for the monomial $n_{i}$. Then, to insure that one gets an ep-deformation after blowing up, one has conditions (iv) for $B^{\prime}$ which are of type

$$
t_{i}+r_{i}=0 \quad \text { for all } i \text { with } n_{i} \notin I_{Q, 0}^{\mathrm{ep}} .
$$

All those monomials are basic ones and $r_{i} \in \mathfrak{m}_{B}^{2}$, therefore the latter conditions are also smooth ones. The ring $B^{\prime}$ is nothing but the quotient of $\widetilde{B}$ by the ideal generated by the above conditions. So, $B^{\prime}$ is a regular local ring and the transform $\eta^{\prime}$ of the deformation $\eta$ is equipolygonal.

Cases (ii) $)_{2}$ and $(\text { ii })_{3}$ : Set $m=: q \cdot m^{\prime}$ with $q$ a power of $p$ and $m^{\prime}$ prime to $p$. Now, the monomials $u z^{m-1}, \ldots, u^{m-1} z, z^{m}$ are all basic monomials, and we can assume that they coincide with $n_{1}, \ldots, n_{m}$ and, hence, $t_{1}, \ldots, t_{m}$ are part of a regular system of parameters for $B$. The leading form $z^{m}+$ $\sum_{i=1}^{m} t_{i} u^{i} z^{m-i}$ should be a pure power of type $(z-a u)^{m}$, so conditions (ii) and (iii) for $B^{\prime}$ can be written in the form $t_{i}=0$ for $i \not \equiv 0$ modulo $q, t_{q}=-m^{\prime} w^{q}$ for a new parameter $w$ in a new regular local ring, and $t_{j} q-(-1)^{j} \cdot\left(\begin{array}{c}m^{\prime} \\ j\end{array}\right) w^{j}=0$ for $j=2, \ldots, m^{\prime}$. Then the quotient ring $\widetilde{B}$ of $B[[w]]$ modulo the ideal generated by the equations given by the above conditions is a regular local ring. The equation $\widetilde{G}$ of the induced deformation $\widetilde{\eta}$ of $\eta$ on $\widetilde{B}$ has the pure power $(z-w u)^{m}$ as leading form. The change $\widetilde{z}=z-w u$ gives rise to

$$
\widetilde{G}(u, \widetilde{z}+w u)=g(u, \widetilde{z})+(w+b) u \frac{\partial g}{\partial z}+\sum_{i=m+1}^{d} t_{i} n_{i}(u, \widetilde{z})+\sum_{j=1}^{\ell} C_{j}(u, \widetilde{z}) g_{j}(u, \widetilde{z})+r(u, \widetilde{z}),
$$

with $C_{j}$ having coefficients in $\mathfrak{m}_{\widetilde{B}}$ and $r$ having coefficients in $\mathfrak{m}_{\widetilde{B}}^{2}$.

In case (ii) $)_{2}$, all of the monomials $n \in I_{R_{Q}}^{\mathrm{ep}} \backslash I_{Q, 0}^{\mathrm{ep}}$ are basic. Conditions (iv) in at the beginning of $\S 8$ are of type

$$
t_{i}+r_{i}=0 \quad \text { for all } i>m \text { and } n_{i} \notin I_{Q, 0}^{\mathrm{ep}},
$$

where $r_{i} \in \mathfrak{m}_{\widetilde{B}}^{2}$ is the coefficient of $n_{i}$ in $r$. The ring $B^{\prime}$ is nothing but the quotient of $\widetilde{B}$ modulo the equations given by the above conditions, so it is a regular local ring. 


\section{A. Campillo, G.-M. Greuel and C. Lossen}

In case (ii) $)_{3}$, all of the monomials $n \in I_{R_{Q}}^{\mathrm{ep}} \backslash I_{Q, 0}^{\mathrm{ep}}$ are basic except the leading monomial $n_{0}$ of $u(\partial g / \partial z)$. Here, conditions (iv) are of type

$$
\begin{aligned}
t_{i}+r_{i} & =0 \quad \text { for all } i>m \text { and } n_{i} \notin I_{Q, 0}^{\mathrm{ep}}, \\
w+b+r^{\prime} & =0
\end{aligned}
$$

with $r_{i}, r^{\prime} \in \mathfrak{m}_{\widetilde{B}}^{2}$. These conditions are, again, smooth, since $w$ is analytically independent from the $t_{i}$ involved in the other conditions and since the linear part of $w+b+r^{\prime}$ involves the parameter $w$ with coefficient 1 . The ring $B^{\prime}$ is again a regular local ring as it is nothing but the quotient of $\widetilde{B}$ modulo the equations given by the above conditions.

In all cases, $\eta^{\prime}$ is the transform of the deformation $\gamma(\eta)$ induced by $\eta$ on $B^{\prime}$. Now, we shall prove that $\eta^{\prime} \in \mathcal{D} e f_{R_{Q^{\prime}}}^{\mathrm{ep}}\left(B^{\prime}\right)$ is ep-versal. For this, we proceed as in case (i).

For this, note that the transformation $\Xi$ associated to the formal blow-up $\widetilde{z}^{\prime}=\widetilde{z} / u$ maps $u^{i} \widetilde{z}^{i}$ to $u^{i+j-m} \widetilde{z}^{j}$ and satisfies $\Xi\left(I_{Q, 0}^{\mathrm{ep}}\right) \not \subset I_{R_{Q^{\prime}}}^{\mathrm{ep}}, \Xi\left(J_{Q, 0}\right) \subset J_{Q^{\prime} R}$, so that it induces a linear map $\bar{\tau}: T_{Q, 0}^{\mathrm{ep}} \rightarrow T_{Q, R}^{\mathrm{ep}}$ which is surjective by the same argument than in case (i). Moreover, as in case (i), the Kodaira-Spencer map $\psi_{\eta^{\prime}}$ is the composite of the Kodaira-Spencer map $\psi_{0}$ of $\gamma \eta$ considered as ep-deformation with respect to auxiliar data in $\mathcal{P}_{0}$ and the linear map $\Xi$. Thus, the surjectivity of $\psi_{\eta^{\prime}}$ will be proved if one checks that $\psi_{0}$ is surjective. However, this follows from the fact that all of the basic monomials $n_{i} \in I_{Q, 0}^{\mathrm{ep}}$ plus the term $\partial g / \partial z$ in case (ii) when $J_{Q, 0} \nsubseteq J_{Q, R}$ appear explicitly in the equation of the deformation $\gamma \eta$ with coefficients $t_{i}=-r_{i} \in m_{B^{\prime}}^{2}$ or $w+b=-r^{\prime} \in m_{B^{\prime}}^{2}$ (for $u(\partial g / \partial z))$. These conditions are part of a regular system of parameters and, therefore, there exist derivatives $\delta_{i} \in T_{B^{\prime}}$ whose images in $T_{Q, 0}^{\mathrm{ep}}$ are equal to $n_{i} \bmod J_{Q, 0}$. This shows the surjectivity of $\psi_{0}: T_{B^{\prime}} \rightarrow T_{Q, 0}^{\mathrm{ep}}$ and, hence, the ep-versality of $\eta^{\prime}$ as required.

Now, assume $s>1$. Then, applying Proposition 7.8, $\eta$ gives rise to an ep-versal deformation $\eta_{T}$ of the multicurve of tangential components of the given curve. Thus, conditions for $B^{\prime}$ are applied simultaneously to all tangential components, so that those which correspond to a single component are smooth, which follows from the case $s=1$. Moreover, they are also smooth all together as the ep-versality for $\eta_{T}$ provides stronger hypothesis than the ep-versality of the individual components. In fact, the ep-versality of $\eta_{T}$ guarantees that all coefficients of the basic monomials of all tangential components form part of a regular system of parameters. This follows from the fact that the surjectivity of the Kodaira-Spencer map $\psi_{\eta_{T}}$ creates independent derivatives for them.

Actually, there are only two types of conditions: those of type $t_{i}+r_{i}=0$ with $r_{i} \in m_{B^{2}}$ (for the coefficient $t_{i}$ of some basic monomial); and those of type $w+b+r^{\prime}=0$ with $b \in m_{B}$ and $w^{q}=t_{i}$ (for $q$ some power of the characteristic and $t_{i}$ the coefficient of some basic monomial). In the latter case, the coefficients of $w$ (in the linear part of the condition) are 1, whereas the coefficients of the other $w^{\prime}$ of similar type which appear are necessarily 0 . This guarantees that the whole set of conditions for $B^{\prime}$ are independent and, therefore, $B^{\prime}$ is smooth.

The ep-versality of the multicurve deformation $\eta^{\prime}$ follows from the independence of the various $t_{i}$ involved in the construction of $B^{\prime}$ and the proof of ep-versality for $s=1$, applied to each component deformation. In fact, from that proof, one deduces that the image of the Kodaira-Spencer map $\psi_{\eta^{\prime}}$ contains every $T_{Q_{j^{\prime}}^{\prime}}^{\mathrm{ep}}$, so $\psi_{\eta^{\prime}}$ is surjective.

Now, we come to the proof of Theorem 6.2.

Proof of Theorem 6.2. Take $\eta=\left(C \rightarrow R_{C}, \tau\right) \in \mathcal{D e f}_{R}^{\mathrm{sec}}(C)$. First, we construct the object $\zeta / \eta$ in the statement.

We may assume that $\eta$ is given by $F(x, y)=\sum_{i+j>0} c_{i j} x^{i} y^{j} \in \mathfrak{m}_{C} C[[x, y]]$. Then, consider the ideal $I^{(0)}$ generated by the element $c_{i j}$ with $i+j<m$, set $\gamma^{(0)}: C \rightarrow C^{(0)}=C / I^{(0)}$ the natural 


\section{EQUISINGULAR DEFORMATIONS IN ARBITRARY CHARACTERISTIC}

map, and $\eta^{(0)}:=\gamma^{(0)}(\eta) \in \mathcal{D} e f_{R}^{\mathrm{ep}}\left(C^{(0)}\right)$. Now, by Definition 7.1 applied to $\eta^{(0)}$ one obtains morphisms $\gamma^{(1)}: C^{(0)} \rightarrow C^{(1)}=C^{\prime}$ and $\eta^{(1)} \in \mathcal{D} f_{\mathbf{R}^{(1)}}^{\mathrm{ep}}\left(C^{\prime}\right)$ which extends $\gamma^{(1)}\left(\eta^{(0)}\right)$. By iterating the construction simultaneously for all points in the infinitesimal neighbourhood of the same order one obtains morphisms $\eta^{(k)} \in \mathcal{D} e f_{\mathbf{R}^{(k)}}^{\text {ep }}\left(C^{(k)}\right)$ such that $\eta^{(k)}$ extends to $\gamma^{(k)}\left(\eta^{(k-1)}\right)$ to points in the $k$ th neighbourhood. Then, we set $C_{\eta}=C^{(h)}, \chi=\gamma^{(h-1)} \circ \cdots \circ \gamma^{(0)}: C \rightarrow C_{\eta}$ and $\zeta=\eta^{(h)}$. Theorem 7.17 shows that one in fact has $\zeta / \eta \in \mathcal{D}$ ef ${ }_{R, \eta}^{\text {wes }}\left(C_{\eta}\right)$.

Now the functoriality of the construction and statement (1) in the theorem follow from the construction and from Remark 8.1 (one can proceed by recurrence on $k$ ) taking into account Theorem 7.17. Statements (2) and (3) follow from the construction and the successive applications of Remark 8.1 and $I$ for the construction of $\zeta / \eta$. Since the extension $C \rightarrow C_{\eta}$ is finite, each condition can increase the codimension at most by one, allowing us to show statement (4) in this way. If, in particular, $\eta$ is versal in $\mathcal{D} e{ }_{R}^{\text {sec }}$, then Proposition 8.2 shows that all of the conditions are smooth and, therefore, $C_{\eta}$ is smooth of codimension equal to the number of conditions which contribute for the construction of $B^{\prime}$.

Proposition 8.2 also allows us to show that each free point $Q$ contributes with $\frac{1}{2} m_{Q}\left(m_{Q}+1\right)-1$ such conditions, whereas each satellite contributes with $\frac{1}{2} m_{Q}\left(m_{Q}+1\right)$. The sum can be extended to any subset of infinitely near points on $R$ which contains all satellite points and those necessary to create them, in particular to $\operatorname{Ess}(R)$ (the minimal subset with the above properties).

Next, let us prove Theorem 6.7 in a parallel way. To prove it, we need some preparations as above.

Let $Q$ be an infinitely near point and fix $\zeta=\zeta_{Q} \in \mathcal{D} e f_{\bar{R}_{Q} \leftarrow R_{Q}}^{\text {ep }}(C)$. Take adapted $U, V$ and consider the tangential components $\zeta_{Q, j} \in \mathcal{D} e f_{\bar{R}_{Q_{j}} \leftarrow R_{Q_{j}}}^{\text {ep }}(C), 1 \leqslant j \leqslant s$. After relabelling, assume that for $1 \leqslant j \leqslant s^{\prime}$ the $j$ th component is not tangent to an exceptional branch and that for $s^{\prime} \leqslant j \leqslant s$ it is tangent.

Consider the following ideals $I_{j} \subset C$. If $j>s^{\prime}, I_{j}=0$. If $j \leqslant s^{\prime}$, fix a concrete $i_{j} \in \Lambda_{Q_{j}^{\prime}}$. For each $i \in \Lambda_{Q_{j}^{\prime}}$, denote by $c_{i}$ the leading term of $\varphi_{Q, C, i}(V) / \varphi_{Q, C, i}(U)$ and set

$$
\varphi_{Q, C, i}(V)-c_{i} \varphi_{Q, C, i}(U)=\sum_{l>m_{i}} c_{i l} t_{i}^{l}
$$

Then $I_{j}$ is the ideal generated by the following elements:

(i) $c_{i}-c_{i_{j}}$ for $i \in \Lambda_{Q_{j}^{\prime}}, i \neq i_{j}$;

(ii) $c_{i l}$ for $i \in \Lambda_{Q_{j}^{\prime}}$ and $m_{i}=m_{e, i}=m_{d, i}<l<m_{i}+m_{i}^{\prime}, m_{i}^{\prime}$ being the multiplicity of the strict transform of the $i$ th branch at $Q_{j}^{\prime}$.

Set $C^{\prime}=C /\left(I_{1}+\cdots+I_{s}\right)$. Then, by construction, the natural morphism $\gamma: C \rightarrow C^{\prime}$ induces a deformation of the parametrization $\zeta^{(1)} \in \mathcal{D e f}_{\mathscr{\mathscr { R }}_{Q, g}}^{\mathrm{ep}}(C)$ which extends the deformation $\gamma(\zeta)$ to the parametric blow-up diagram $\overline{\mathscr{R}}_{Q, g}(C)$.

Remark 8.3. The above construction has the following universal property: For any map $\chi: C \rightarrow A$ and an extension of $\chi(\zeta)$ to an ep-deformation $\xi$ of $\overline{\mathscr{R}}_{Q, g}$ there exists a unique map $\chi^{\prime}: C^{\prime} \rightarrow A$ such that $\chi^{\prime} \circ \gamma=\chi$ and $\xi=\chi^{\prime}\left(\zeta^{(1)}\right)$. In fact, this property follows from the fact that the analogous elements to elements (i) and (ii) in $A$ have to be zero because of the existence of $\xi$. Note also that for $j>s$, no condition is required (in concordance with the fact that $I_{j}=0$ ).

Now, denote by $\zeta^{\prime}$ the deformation of the multicurve $\overline{\mathscr{R}}_{Q_{i}}^{\prime}$ obtained from the data $\left(\overline{\mathscr{R}}_{Q, g}, \zeta^{(1)}\right)$ by deleting $Q$ from the diagram and the assignment of $\gamma(\zeta)$ at $Q$ to the list of deformations defining $\zeta^{(1)}$. 


\section{A. Campillo, G.-M. Greuel and C. Lossen}

Proposition 8.4. With the assumptions and notation as above, if $\zeta \in \mathcal{D} e \frac{\mathrm{ep}}{\bar{R}_{Q} \leftarrow Q}(B)$ is an ep-versal deformation of the parametrization with smooth base $B$, then the deformation $\zeta^{\prime} \in \mathcal{D} e f_{\mathscr{R}^{\prime}}^{\text {ep }}{ }_{Q, g}\left(B^{\prime}\right)$ is ep-versal and its base $B^{\prime}$ is also smooth.

Proof. First assume $s=1$, and consider the two possibilities: (i) $s^{\prime}=0$; (ii) $s^{\prime}=1$.

Case (i): Assume, for instance, $m_{e, i}<m_{d, i}$ for all $i \in \Lambda_{Q}$ (without loss of generality). One has $e=1<d$ at $Q$ and $e^{\prime}=1 \leqslant d^{\prime}=d-1$ at $Q^{\prime}$. Then $\sigma^{\prime}$ is nothing but the intersection of $E_{Q^{\prime}, A}$ and $D_{Q^{\prime}, A}$ and $\zeta^{\prime}$ is equipolygonal with $B^{\prime}=B$. The result follows from the fact that the KodairaSpencer map $\psi_{\zeta^{\prime}}$ is nothing but the composite of two surjective maps, namely the Kodaira-Spencer map $\psi_{\zeta}$ and the linear map

$$
\bar{\Xi}: T_{\bar{R}_{Q} \leftarrow R_{Q}}^{\mathrm{ep}} \rightarrow T_{\bar{R}_{Q^{\prime}} \leftarrow R_{Q^{\prime}}}^{\mathrm{ep}}
$$

induced by $\Xi\left(a_{1}, \ldots, a_{r^{\prime}}, b_{1}, \ldots, b_{r^{\prime}}\right)=\left(a_{1}, \ldots, a_{r^{\prime}}, b_{1} / a_{1}, \ldots, b_{r^{\prime}} / a_{r^{\prime}}\right)$ where $r^{\prime}=\# \Lambda_{Q}$.

Case (ii): One has $d=e=1, m_{i}=m_{i, e}=m_{i, d}$. Take adapted $U, V$ with $u=U\left(\bmod \mathfrak{m}_{B}\right)$ transversal to $Q \rightarrow \bar{R}_{Q}$. Since $Q^{\prime}$ is free and $U$ an equation of $E_{Q^{\prime}, B}$ at $Q_{B}^{\prime}$, one can replace $U, V$ by non-adapted $U, Z=V+\lambda U, \lambda \in K$, such that $z=Z\left(\bmod \mathfrak{m}_{B}\right)$ is tangent to the branches of $Q \rightarrow \bar{R}_{Q}$.

Thus, for $i \in \Lambda_{Q}=\Lambda_{Q^{\prime}}$ one has $c_{i}=\lambda+b_{i}$ where $b_{i} \in \mathfrak{m}_{B}$ is the leading term of $\varphi_{Q, B, i}(Z) /$ $\varphi_{Q, B, i}(U)$ and

$$
\varphi_{Q, B, i}(Z)-b_{i} \varphi_{Q, B, i}(U)=\varphi_{Q, B, i}(V)-c_{i} \varphi_{Q, B, i}(U)=\sum_{i>m_{i}} c_{i l} t_{i}^{l} .
$$

One also has ord $\varphi_{Q, i}(Z) \geqslant m_{i}+m_{i}^{\prime}$ with equality if $m_{i}^{\prime}<m_{i}$.

Consider the vector space $\bar{T}_{Q, 0}^{\mathrm{ep}}=\bar{I}_{Q, 0}^{\mathrm{ep}} / \bar{J}_{Q, 0}^{\mathrm{ep}}$, where:

(1) $\bar{I}_{Q, 0}^{\mathrm{ep}}=\bigoplus_{i \in \Lambda_{Q}} \overline{\mathfrak{m}}_{i}^{m_{i}} \oplus \overline{\mathfrak{m}}_{i}^{m_{i}+m_{i}^{\prime}} \subset I_{\bar{R}_{Q} \leftarrow R_{Q}}^{\mathrm{ep}}$;

(2) $\bar{J}_{Q, 0}=\overline{\mathfrak{m}}(\dot{u}, \dot{z})+\mathfrak{m} \oplus \mathfrak{m}_{0}$, with $\mathfrak{m}_{0}=\left(u^{2}, z\right) \subset \mathfrak{m}=\mathfrak{m}_{Q} \subset R_{Q}$.

Note that $J_{Q, \bar{R} \leftarrow R} / \bar{J}_{Q, 0}$ is generated by $(0, u)$ as vector space. Hence, the obvious linear map $\Phi$ : $\bar{T}_{Q, 0}^{\mathrm{ep}} \rightarrow T_{Q, \bar{R} \leftarrow R}^{\mathrm{ep}}$ is injective as one has $J_{Q, \bar{R} \leftarrow R} \cap I_{0}^{\mathrm{ep}}=J_{0}$.

Now, one has $B^{\prime}=B / I$ where $I$ is generated by the elements of $\mathfrak{m}_{B}$ given by: (i) $b_{i}-b_{i_{1}}, i, i_{1} \in$ $\Lambda_{Q}=\Lambda_{Q}, i \neq i_{1}, i_{1}$ fixed; (ii) $c_{i l}, i \in \Lambda_{Q}$ and $m_{i}<l<m_{i}+m_{i}^{\prime}$. Let $F$ be the set of indices $(i, l)$ with $i \in \Lambda_{Q}$ and $m_{i}<l<m_{i}+m_{i}^{\prime}$ or $l=m_{i}, i \neq i_{1}$. For each $(i, l) \in F$, let $z_{i, l}$ be the element in $I_{\bar{R}_{Q} \leftarrow R_{Q}}^{\mathrm{ep}}$ whose $j$ th component modulo $J_{Q, R \rightarrow \bar{R}}$ is $\left(0, t_{i}^{m_{i}}\right)$. Then, the set consisting of the elements $z_{i, l}$ with $(i, l) \in F$ gives a set of linearly independent classes in $T_{Q, \bar{R} \rightarrow R}^{\mathrm{ep}}$. Since the Kodaira-Spencer map $T_{A} \rightarrow T_{Q, \bar{R} \leftarrow R}^{\mathrm{ep}}$ is surjective, there exist derivations $\delta_{i, l} \in T_{A}$ whose images in $T_{Q, R \rightarrow \bar{R}}^{\mathrm{ep}}$ are the classes of the corresponding $z_{i, l}$.

Set $\varphi_{Q, B, i}(Z)-b_{i_{1}} \varphi_{Q, B, i}(U)=\sum_{l \geqslant m_{i}} s_{i l} t_{i}^{l}$. If $\varphi_{Q, B, i}(U)=\sum_{l \geqslant m_{i_{1}}} u_{i l} t_{i_{1}}^{l}$ one has $s_{i_{1} m_{i}}=0$, $s_{i, m_{i}}=\left(b_{i}-b_{i_{1}}\right) u_{i m_{i}}$ for $i \neq i_{1}$, and $s_{i l}=c_{i l}+\left(b_{i}-b_{i_{1}}\right) u_{m_{i_{1}}}$ for $l>m_{i}$. Since $u_{i m_{i}} \notin \mathfrak{m}_{B}$ one has that the ideal $I$ is also generated by the elements $s_{i l}$ with $(i, l) \in F$.

Now, by construction, one has $\delta_{i l}\left(s_{i^{\prime} l^{\prime}}\right)=0$ if $(i, l) \neq\left(i^{\prime}, l^{\prime}\right)$ and $\delta_{i l}\left(s_{i l}\right)=1$, so the elements $s_{i l} \in \mathfrak{m}_{B}$ are linearly independent modulo $\mathfrak{m}_{B}^{2}$ and, hence, they are part of a regular system of parameters of $B$. This shows that $B^{\prime}$ is smooth.

It remains to check that $\zeta^{\prime}$ is ep-versal. For this, note that one has

$$
T_{B^{\prime}}=\left\{\delta \in T_{B} \mid \delta\left(s_{i l}\right)=0 \text { for all }(i, l) \in F\right\} .
$$

Hence, one has a induced linear map $\Psi_{0}: T_{B^{\prime}} \rightarrow \bar{T}_{Q, 0}^{e p}$ which is nothing but the Kodaira-Spencer map for $\gamma(\zeta)$ considered as ep-deformation with respect to the data $\mathcal{P}_{0}$. Since $\Phi \circ \Psi_{0}$ is equal to the 


\section{EQUISINGULAR DEFORMATIONS IN ARBITRARY CHARACTERISTIC}

restriction of $\Psi_{\zeta}$ to $T_{B^{\prime}}$, $\Phi$ is injective and $\Psi_{\zeta}$ surjective, it follows that $\Psi_{0}$ is surjective. On the other hand, considering $\gamma(\zeta)$ ep-deformation with respect to $\mathcal{P}_{0}$, the situation is exactly the same as in case (i), so that, in particular, the analogous map $\bar{\Xi}_{0}$ to $\bar{\Xi}$ is surjective. It follows that $\Psi_{\zeta^{\prime}}=\bar{\Xi}_{0} \circ \Psi_{0}$ is surjective.

For $s>1$, taking into account Theorem 7.17, the situation is completely analogous to that of Proposition 8.2. The smoothness of $B^{\prime}$ and ep-versality of the multicurve deformation $\zeta^{\prime}$ follows in the same way. We do not repeat the arguments here.

Now, the proof of Theorem 6.7 follows in a similar way as for Theorem 6.2.

Proof of Theorem 6.7. Take $\zeta=\left(\varphi_{C}, \sigma, \bar{\sigma}\right) \in \mathcal{D}$ ef $\frac{\sec }{R \leftarrow P}(C)$. First, we construct the ideal $I_{\zeta}$ in the statement.

Assume that $\zeta$ is given by $X_{i}(t)=\sum_{j>0} a_{i j} t^{j}, Y_{i}(t)=\sum_{j>0} b_{i j} t^{j}, i \in \Lambda$ with $a_{i j}, b_{i j} \in \mathfrak{m}_{C}$. Then, consider the ideal $I^{(0)}$ generated by the elements $a_{i j}, b_{i j}$ with $0<j<m_{i}$ and $i \in \Lambda$. Set $C^{(0)}:=$ $C / I^{(0)}, \gamma^{(0)}$ the natural map, and $\zeta^{(0)}:=\gamma^{(0)}(\zeta) \in \mathcal{D} e f_{R \rightarrow \bar{R}}^{\mathrm{ep}}\left(C^{(0)}\right)$. Now, the successive application of the construction for $C^{\prime}$ (starting from $\zeta(0)$ ) gives rise to a sequence of ideals $I^{(0)} \subset I^{(1)} \subset \cdots \subset \cdots$ and deformations $\zeta^{(k)} \in \mathcal{D} e f_{\overline{\mathbf{R}}^{(k)}}^{\mathrm{ep}}\left(C^{(k)}=C / I^{(k)}\right)$ such that, for each $k, \zeta^{(k)}$ extends to the $k$ th-order neighbourhood points the deformation $\gamma^{(k)}\left(\zeta^{(k-1)}\right), \gamma^{(k)}=C^{(k-1)} \rightarrow C^{(k)}$ being the obvious map. Then, set $I_{\zeta}=I^{(h)}, C_{\eta}=C / I_{\zeta}=C^{(h)}, \pi: C \rightarrow C_{\zeta}$ the natural map, and $\zeta=\pi(\zeta)$. Note that $\zeta \in \mathcal{D} e f \frac{\mathrm{es}}{R \leftarrow P}\left(C_{\zeta}\right)$ as it is the image of $\zeta^{(h)} \in \mathcal{D} e f_{\overline{\mathbf{R}}}^{\mathrm{ep}}\left(C_{\zeta}\right)$ by the isomorphism in Theorem 7.17.

Statement (1) follows from the construction, Theorem 7.17 and Remark 8.3. Statements (2) and (3) follow from the construction and successive applications of Proposition 8.4. The integer $\operatorname{con} \frac{\mathrm{es}}{R \leftarrow P}$ is the number of conditions used in the successive applications to form $I^{(0)}$ for the construction of $I_{\zeta}$, so this number bounds the codimension of the equisingular stratum, showing statement (2). If $\zeta$ is versal for $\mathcal{D} e f \frac{\mathrm{sec}}{\bar{R} \leftarrow P}$, then the successive applications of Proposition 8.4 show that all of those conditions are smooth and transversal, hence, $C_{\zeta}$ is smooth and of codimension con $\overline{\mathrm{es}} \leftarrow P^{\text {. }}$

The construction of $I^{(0)}$ and the successive applications of Proposition 8.4 starting from $\zeta$ versal

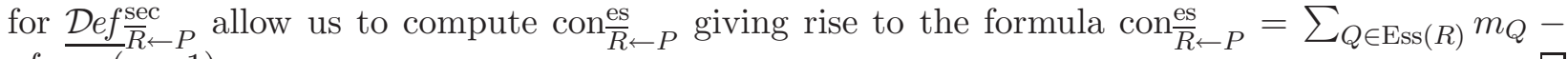
ef $f_{R}-(r-1)$.

\section{Geometry of equisingular strata}

In this section, we study the geometry and show relations among the different equisingularity strata and objects related to them. In particular, we prove that the dimension of the weak equisingularity stratum is related to the terms in the equisingularity exact sequences of $\S 5$.

Let $\eta_{s u}=\eta=\left(B \rightarrow R_{B}, \tau\right) \in \mathcal{D} e f_{R}^{\mathrm{sec}}(B)$ be a semiuniversal deformation in $\mathcal{D} e f_{R}^{\mathrm{sec}}$. Then $B$ is a regular local ring. For the weakly equisingular deformation $\zeta / \eta$ of $R$ based in $\eta$, one has that $B_{\eta}$ is a regular local ring, the map $B / I_{\eta} \rightarrow B_{\eta}$ induced by $B \rightarrow B_{\eta}$ is finite and the weak equisingularity stratum $S_{R}^{\text {wes,sec }}=\operatorname{Spec}\left(B / I_{\eta}^{\text {wes }}\right)$ is irreducible with $\operatorname{dim} S_{R}^{\text {wes,sec }}=\operatorname{dim} \operatorname{Spec}\left(B_{\eta}\right)=\operatorname{dim} B_{\eta}$.

TheOREM 9.1. With the above assumptions and notation from $\S 5$,

$$
\operatorname{dim} S_{R}^{\text {wes, sec }}=\operatorname{dim} B_{\eta}=\operatorname{dim} T_{R}^{1, \text { es }}+\operatorname{dim} T_{\bar{R} / R}^{1, \mathrm{es}}=\operatorname{dim} T_{\bar{R} \leftarrow R}^{1, \mathrm{es}}+\operatorname{dim} M_{R}^{\mathrm{sec}} .
$$

Proof. From Theorems 6.2 and 6.7 one has

$$
\begin{aligned}
\operatorname{dim} B_{\eta} & =\tau_{R}^{\mathrm{sec}}-\frac{1}{2} \sum_{Q \in \operatorname{Ess}(R)} m_{Q}\left(m_{Q}+1\right)+e f_{R}, \\
\operatorname{dim} T_{\bar{R} \leftarrow R}^{1, \mathrm{es}} & =\tau_{\bar{R} \leftarrow P}^{1, \mathrm{sec}}-\sum_{Q \in \operatorname{Ess}(R)} m_{Q}+e f_{R}+(r-1) .
\end{aligned}
$$




\section{A. Campillo, G.-M. Greuel and C. Lossen}

On the other hand, from Lemma 5.4 one has

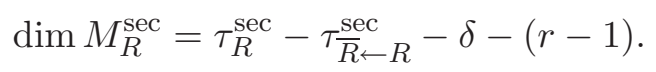

Finally, one has the well-known formula $\delta=\frac{1}{2} \sum_{Q \in \operatorname{Ess}(R)} m_{Q}\left(m_{Q}-1\right)$. It follows that $\operatorname{dim} B_{\eta}=$ $\operatorname{dim} T_{\bar{R} \leftarrow R}^{1, \text { es }}+\operatorname{dim} M_{R}^{\text {sec }}=\operatorname{dim} T_{R}^{1, \text { es }}+\operatorname{dim} T_{\bar{R} / R}^{1, \text { es }}$, the last equality being a consequence of the exact sequence in Proposition 5.5.

Since the base spaces of the semiuniversal deformations for $\underline{\mathcal{D} e f}{ }_{R}^{\text {es }}$ and $\underline{\mathcal{D} e f} \frac{\mathrm{es}}{\bar{R}} \leftarrow R$ are smooth, the above theorem shows dimensional relations between the different semiuniversal equisingular deformations and equisingular strata. Next, we will see how the geometric nature of these objects can also be understood in terms of the object $\zeta / \eta$.

For this, we first give a technical result. Consider a given diagram of the type

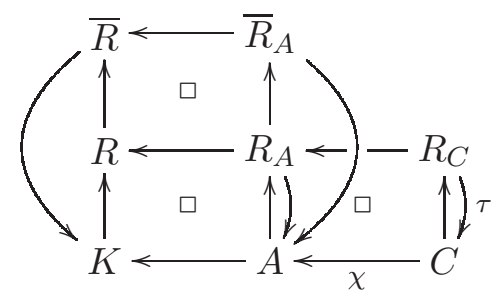

that is, a weakly wes of $R$ based in $\left(C \rightarrow R_{C}, \tau\right)$, where $\chi$ is finite and injective. Denote by $K(\chi)$ the kernel of the tangent map $T(\chi): T_{A} \rightarrow T_{C}$. Further denote by $T_{R}^{1 \text {,wes,sec }} \subset T_{R}^{1, \text { sec }}$ the Zariski tangent space to the weak equisingularity stratum $S_{R}^{\text {wes,sec }}$. One obviously has $T_{R}^{1, \text { es }} \subset T_{R}^{1 \text {,wes,sec }} \subset \mathbb{T}_{R}^{1, \text { sec }}$. Note that, if one fixes $\left(C \rightarrow R_{C}, \tau\right)$, then the weakly equisingular deformation based on it is nothing but a diagram as above which satisfies a universal property among such diagrams.

Proposition 9.2. For a given diagram as above, one has an induced commutative diagram of vector spaces

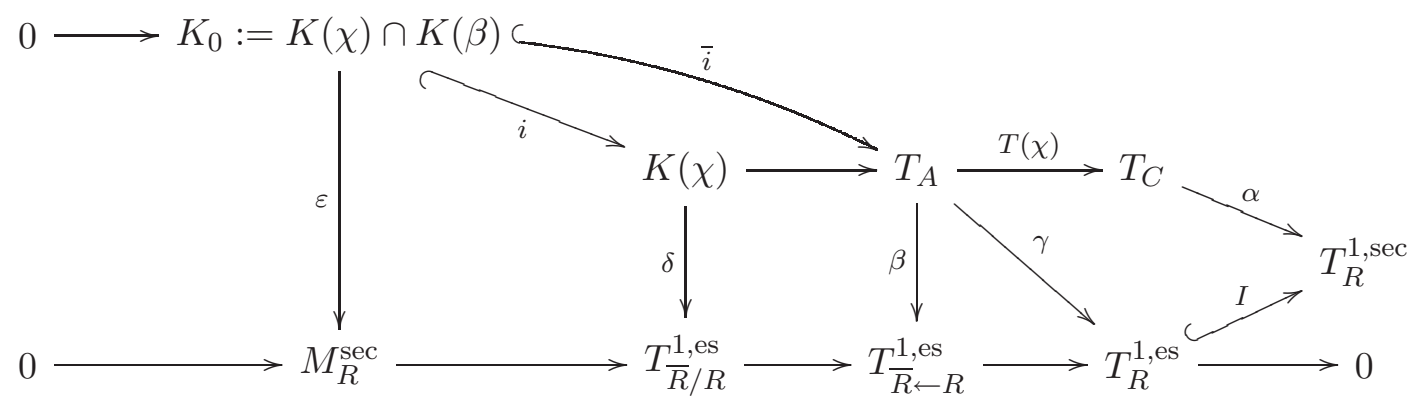

where all linear maps $\alpha, \beta, \gamma, \delta, \varepsilon$ only depend on the given diagram; the maps $\alpha, \gamma$ depend only on $\chi(\eta)$ and $\beta, \delta, \varepsilon$ on the fixed es-deformation of the parametrization for $\chi(\eta)$. The bottom row is nothing but the exact sequence in Proposition 5.5 and the other maps $I, i, \bar{i}$, are inclusions.

Proof. (1) The map $\alpha$ is given by the fact that $\eta \in \mathcal{D} e f_{R}^{\mathrm{sec}}(C)$.

(2) The map $\beta$ is given by the fact that $\chi(\eta)$ plus the chosen deformation of the parametrization is an element of $\mathcal{D} e f \frac{\mathrm{es}}{R \leftarrow R}(A)$.

(3) The map $\gamma$ is the composite of $T_{\bar{R} \leftarrow R}^{1, \text { es }} \rightarrow T_{R}^{1, \text { es }}$ with $\beta$. One has $I \circ \gamma=\alpha \circ(T(\chi))$.

(4) Take a vector $v \in K(\chi) \subset T_{A}$. Then $\delta(v)$ is defined as follows. Since $v: A \rightarrow k[\varepsilon]$ is a $k$-algebra morphism such that $v \circ \chi=0$, one has that the induced deformation on $k[\varepsilon]$ by the base change $v$ is trivial, therefore, defines an element of $T_{\bar{R} / R}^{1, \text { es }}$ (since at the level of $A$ one has a given deformation of the parametrization too). This element of $T_{\bar{R} / R}^{1, \mathrm{es}}$ is just $\delta(v)$. 


\section{EQUISINGULAR DEFORMATIONS IN ARBITRARY CHARACTERISTIC}

(5) If $v \in K_{0}$, then $\delta(v)$ is an element of $T_{\bar{R} / R}^{1, \text { es }}$ whose image in $T_{R \rightarrow \bar{R}}^{1, \text { es }}$ is zero (as $v \in \operatorname{ker}(\beta)$ ), so one has $\delta(v) \in M_{R}^{\mathrm{sec}}$. The map $\varepsilon$ is nothing but the restriction of $\delta$ to $K_{0}$ taking as target $M_{R}^{\mathrm{sec}}$ instead of $T_{\bar{R} / R}^{1, \mathrm{es}}$.

Remark 9.3. Because of the universal property of the weak stratum one has that the image under $\alpha$ of the subspace $T_{C / \operatorname{ker}(\chi)}$ of $T_{C}$ is in $T_{R}^{1, \text { wes,sec }}$.

Now, consider the particular case of the diagram

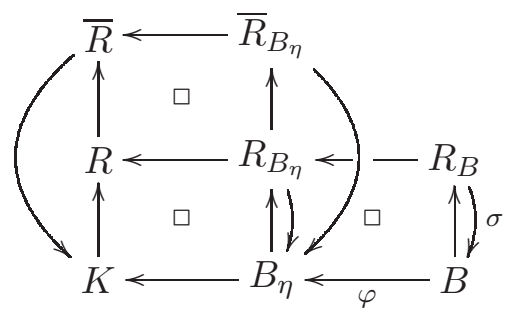

given by the weakly equisingular deformation $\zeta / \eta$ based on a semiuniversal deformation $\eta=(B \rightarrow$ $\left.R_{B}, \sigma\right)$ in $\mathcal{D} e f_{R}^{\mathrm{sec}}$. Since $\eta$ is semiuniversal, it follows from the universal property of $\zeta / \eta$ that for any diagram $(\mathrm{d})$ there is a diagram map from $\left(\mathrm{d}_{\mathrm{su}}\right)$ to $(\mathrm{d})$. In other words, any weak es-deformation with respect to any given deformation can be induced (not in a unique way) from $\zeta / \eta$. Moreover, one has the following particular situation.

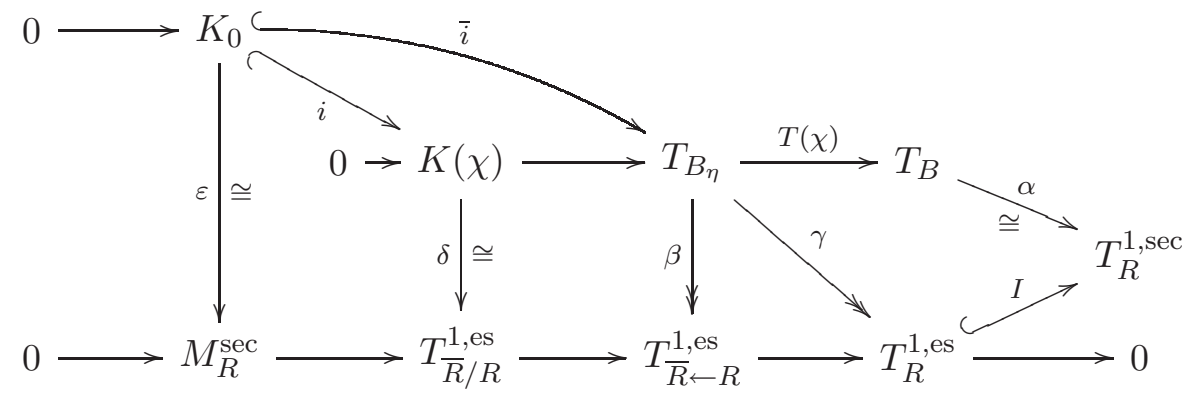

Hence, one has the following lemma.

LEMma 9.4. With the above assumptions and notation, one has the following properties for the object $\zeta / \eta$ :

(1) $\alpha$ is an isomorphism;

(2) $\gamma$ is surjective;

(3) $\delta$ is an isomorphism;

(4) $\operatorname{ker}(\beta) \subset \operatorname{ker}(\varphi)$, so $K_{0}=\operatorname{ker}(\beta)$;

(5) $\varepsilon$ is an isomorphism;

(6) $\beta$ is surjective.

Proof. Statement (1) is obvious from the semiuniversality of $\eta$ with respect to the functor $\frac{\mathcal{D} e f}{R}{ }_{R}^{\mathrm{sec}}$. Statement (2) (respectively, (6)) follows from the fact that every vector in $T_{R}^{1}$ es (respectively, $\bar{T}_{\bar{R} \leftarrow R}^{1, \text { es }}$ ) gives rise to a concrete diagram (d) with $A=k[\varepsilon]$, so the existing diagram map from $\left(\mathrm{d}_{\mathrm{su}}\right)$ to $(\mathrm{d})$ shows that $\gamma$ (respectively, $\beta$ ) is surjective. The same argument, applied to vectors in $T_{\bar{R}}^{1, \text { es }} /$ shows that also $\delta$ is surjective. Since, from Theorem 9.1, $\operatorname{dim} K(\chi)=\operatorname{dim} T_{\bar{R}}^{1, \mathrm{es}}$, statement (3) follows. Moreover $\operatorname{dim} K_{0}=\operatorname{dim} M_{R}^{\text {sec }}$, so statement (5) follows from statement (3).

We now arrive at the theorem which gives the geometric counterpart of Theorem 9.1. 


\section{A. Campillo, G.-M. Greuel and C. Lossen}

THEOREM 9.5. With the above assumptions and notation, the following hold for the object $\zeta / \eta$.

(A) There exists the following natural diagram of vector spaces with three exact sequences.

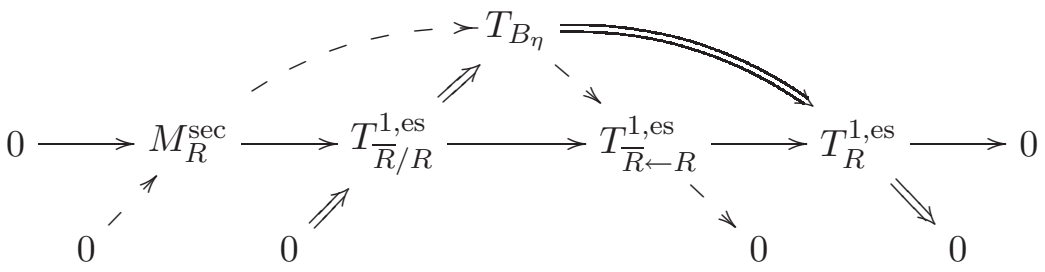

(B) The image of the subspace $T_{\bar{R} / R}^{1, \mathrm{es}}$ (respectively, $M_{R}^{\mathrm{sec}}$ ) in $T_{B_{\eta}}$ is a well-defined subspace which represents the tangent space to the trivial (respectively, parametrically trivial) subfamilies of $\zeta$.

(C) There exist smooth subschemes of $\operatorname{Spec}\left(B_{\eta}\right)$ such that the induced family of $\zeta$ restricted to them gives a semiuniversal deformation for $\underline{D e f}_{R}^{\text {es }}$. Such subschemes are exactly those smooth ones which are complementary to the image of $T_{\bar{R} / R}^{1, \mathrm{es}}$, so they may have different tangent spaces. Their images in $S_{R}^{\mathrm{wes}, \mathrm{sec}}$ are also smooth, all share the same tangent space, and the induced deformation of $\eta$ on them is semiuniversal for $\underline{\mathcal{D} e f}{ }_{R}^{\mathrm{es}}$. Moreover, $S_{R}^{\mathrm{wes}, \mathrm{sec}}$ is nothing but the Zariski closure of the union of those smooth subschemes.

(D) There exist smooth subschemes of $\operatorname{Spec}\left(B_{\eta}\right)$ such that the induced family of $\zeta$ restricted to them provides a semiuniversal deformation for $\mathcal{D} e f \frac{\mathrm{es}}{R \leftarrow R}$. These subschemes are exactly all those smooth ones which are transversal to the image of $M_{R}^{\text {sec }}$, so they may have different tangent spaces.

Proof. Part (A) follows from Lemma 9.4, using $\delta^{-1}$ (respectively, $\varepsilon^{-1}$ ) to define the linear map $T_{\bar{R} / R}^{1, \text { es }} \rightarrow T_{B_{\eta}}$ (respectively, $M_{R}^{\text {sec }} \rightarrow T_{B_{\eta}}$ ) in the theorem. Part (B) is obvious. Parts (C) and (D) partially follow from the fact that a semiuniversal deformation $\eta$ (respectively, $\zeta$ ) for the functor $\underline{\mathcal{D}}_{R}^{\mathrm{es}}$ (respectively, $\underline{\mathcal{D} e f} \overline{\mathrm{es}} \leftarrow R$ ) gives rise to a diagram

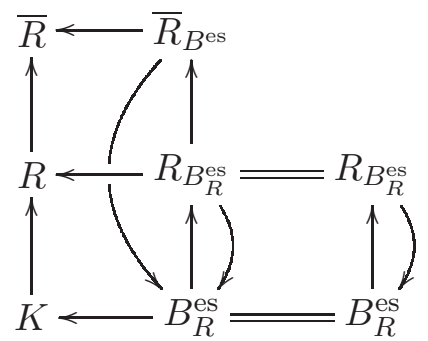

respectively

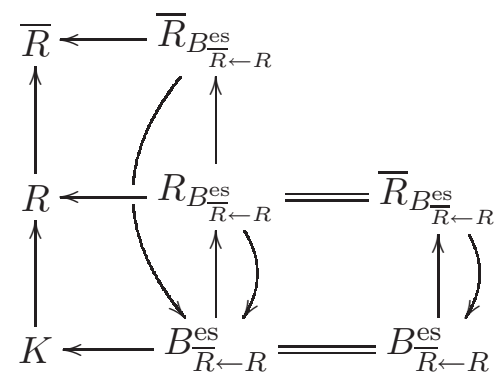

$(\mathrm{d} \bar{R} \leftarrow R)$

so $\left(d_{R}^{\text {es }}\right)$ (respectively, $\left.\left(d_{\bar{R} \leftarrow R}^{\text {es }}\right)\right)$ are images of $\left(d_{\text {su }}\right)$ by a (not unique) diagram map. This allows us to consider $B_{R}^{\text {es }}$ (respectively, $B_{\bar{R}}^{\text {es }}$ ) ) as isomorphic to concrete smooth subspaces of $B_{\eta}$ satisfying the properties stated in part (C) (respectively, (D)). For the case of $\underline{D}_{f} f_{R}^{\text {es }}$ the space $B_{R}^{\text {es can also }}$ be seen as isomorphic to a smooth subspace of $B_{R}^{\mathrm{sec}}=B$. The fact that the diagram map is not 


\section{EQUISINGULAR DEFORMATIONS IN ARBITRARY CHARACTERISTIC}

unique explains that one may have several copies of $B_{R}^{\text {es }}$ (respectively, $B_{\bar{R} \leftarrow R}^{\text {es }}$ ) inside $B_{\eta}$. All copies may be tangent or transversal to each other, but they need to be transversal to the image of $T_{\bar{R}}^{1, \text { es }}$ (respectively, $M_{R}^{\text {sec }}$ ) inside $T_{B_{\eta}}$. However, since $T_{R}^{1, \text { es }}$ is a well-defined vector subspace of $T_{R}^{1, \text { sec }}$, the image of the copies of $B_{R}^{\text {es }}$ inside $B_{R}^{\text {sec }}$ share all $T_{R}^{1, \text { es }}$ as their tangent space. Note that, in Remark 4.3(6), such a copy was considered a candidate for an es-stratum. All these strata need to be inside the weak equisingular stratum $S_{R}^{\text {wes,sec }}$.

The remainder of parts (C) and (D) follow from general arguments applied to this particular situation. In fact, the deformation $\xi$ on $B_{\eta}$ which is based in $\eta$ is versal for both $\frac{\mathcal{D} e f}{R}$ es and $\underline{\mathcal{D} e f} \frac{\mathrm{es}}{\bar{R} \leftarrow R}$ (if one forgets the involved deformation of $\bar{R}$ in the second case). Thus, one has a submersion $\operatorname{Spec}\left(B_{\eta}\right) \rightarrow \operatorname{Spec}\left(B_{R}^{\mathrm{es}}\right)$ (respectively, $\left.\operatorname{Spec}\left(B_{\eta}\right) \rightarrow \operatorname{Spec}\left(B_{\bar{R} \leftarrow R}^{\mathrm{es}}\right)\right)$ such that:

(i) $\xi$ is isomorphic to the pull-back of the semiuniversal equisingular deformation (respectively, equisingular deformation of the normalization) on $\operatorname{Spec}\left(B_{R}^{\mathrm{es}}\right)\left(\operatorname{respectively,~} \operatorname{Spec}\left(B_{\bar{R} \leftarrow R}^{\mathrm{es}}\right)\right.$ ),

(ii) the kernel of the tangent map is the image of $T_{\bar{R} \leftarrow R}^{1 \text {,es }}$ (respectively, $M_{R}^{\text {sec }}$ ).

Then if $\widetilde{S}$ is a smooth subscheme of $\operatorname{Spec}\left(B_{\eta}\right)$ which is complementary to the image of $T_{\bar{R}}^{1, \text { es }} / R$ (respectively, $M_{R}^{\mathrm{sec}}$ ), the deformation induced by $\xi$ on it is isomorphic, via the induced isomorphism $\widetilde{S} \rightarrow \operatorname{Spec}\left(B_{R}^{\text {es }}\right)$ (respectively, $\left.\widetilde{S} \rightarrow \operatorname{Spec}\left(B_{\bar{R} \leftarrow R}^{\text {es }}\right)\right)$, to the pull-back of the semiuniversal equisingular deformation. So the induced deformation of $\xi$ on $\widetilde{S}$ is itself semiuniversal equisingular. Moreover, if $\widetilde{S}$ is complementary to the image of $T_{\bar{R}}^{1, \text { es }}$, then the image $S^{\prime \prime}$ of $\widetilde{S}$ in $S=\operatorname{Spec}(B)$ is also a smooth subscheme of $S$ isomorphic to $\widetilde{S}$, as the tangent map to $\widetilde{S} \rightarrow S$ is injective. It follows that the deformation induced by $\eta$ on $S^{\prime \prime}$ is again semiuniversal for $\underline{\mathcal{D} e f}{ }_{R}^{\text {es }}$. Finally, by construction, it is clear that $S_{R}^{\text {wes,sec }}$ is the Zariski closure of the union of the subspaces $S^{\prime \prime}$ as above.

This completes the proof of the theorem.

The following result characterizes when the weak equisingularity stratum and the strong equisingularity stratum coincide. In fact, it characterizes numerically when a unique strong equisingularity stratum exists.

COROLlary 9.6. The following conditions for a plane curve singularity are equivalent:

(i) $T_{\bar{R} / R}^{1, \mathrm{es}}=0$;

(ii) $S_{R}^{\mathrm{wes}, \mathrm{sec}}$ and $\operatorname{Spec}\left(B_{R}^{\mathrm{es}}\right)$ have the same dimension;

(iii) $S_{R}^{\text {wes,sec }}$ and $\operatorname{Spec}\left(B_{R}^{\mathrm{es}}\right)$ are isomorphic;

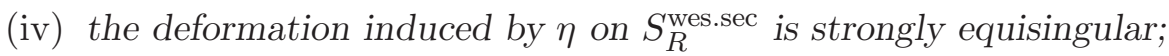

(v) the morphism $\operatorname{Spec}\left(B_{\eta}\right) \rightarrow S^{\text {wes,sec }}$ is an isomorphism;

(vi) there is only one smooth subscheme of $S=\operatorname{Spec}(B)$ such that the deformation induced by $\eta$ on it is semiuniversal for $\underline{\mathcal{D} e f}{ }_{R}^{\mathrm{es}}$;

(vii) there is a unique largest subscheme of $S$ such that the deformation induced by $\eta$ on it is strongly equisingular.

Proof. (i) $\Rightarrow$ (ii) follows from Theorem 9.1 , (ii) $\Rightarrow$ (iii) and (iii) $\Rightarrow$ (iv) follow from the fact that $S_{R}^{\text {wes }}$ contains subschemes isomorphic to $\operatorname{Spec}\left(B_{R}^{\text {es }}\right)$. Statements (iv) and (v) are equivalent, since in both cases the inclusion $S_{R}^{\text {wes,sec }} \hookrightarrow \operatorname{Spec}(B)$ provides a universal object for the category $\mathcal{D} e f_{R, \eta}^{\text {es }}$. Finally, from condition (iv) it follows that $\operatorname{dim} S_{R}^{\text {wes }} \leqslant \operatorname{dim} T_{K, R}^{1, \text { es }}$, so $\operatorname{dim}_{K} T_{\bar{R} / R}^{1}=0$. The equivalence of conditions (i) $-(\mathrm{v})$ with conditions (vi) and (vii) follows from Theorem $9.5(\mathrm{C})$. 


\section{A. Campillo, G.-M. Greuel and C. Lossen}

Example 9.7. From the above results one can review the cases in Example 4.4. In particular, in the four cases the subscheme $S^{\prime}$ can be shown to be nothing but the weak equisingularity stratum $S_{R}^{\mathrm{wes}, \mathrm{sec}}$.

Case 1: The embedded resolution consists of the blow-up of one point of multiplicity $2 p$ and $2 p$ points of multiplicity 1, with ef $f_{R}=2$, so one has $\operatorname{dim} S_{R}^{\text {wes }}=\operatorname{dim} T_{R}^{1, \text { es }}-\left(2 p^{2}+3 p-2\right)=1+(p-1)(p-2)$. By Theorem 9.1 and Example 5.6 one has $\operatorname{dim} B_{R}^{\mathrm{es}}=(p-1)(p-2)$ and $\operatorname{dim} B_{\bar{R}}^{\mathrm{es}}=1+(p-1)(p-2)$. In particular, $B_{\eta}$ is isomorphic to $B \frac{\mathrm{es}}{\bar{R} \leftarrow R}$ and each $S_{h}$ is isomorphic to $\operatorname{Spec}\left(B_{R}^{\mathrm{es}}\right)$. The computation of $B_{\eta}$ gives rise to

$$
\begin{array}{ll}
p \neq 2: & u_{i, j}=0 \text { for }(i, j) \in D \backslash\{(2 p, 0),(p, p)\} \\
& 2 u_{2 p, 0}-u_{p, p}^{2}=0 \\
p=2: & u_{i, j}=0 \text { for }(i, j) \in D \backslash\{(4,0)\} \\
& u_{4,0}=w_{4,0}^{4} .
\end{array}
$$

By eliminating $w_{p p}$ and $w_{40}$ one obtains the equations for $S_{R}^{\text {wes,sec }}$ which are exactly those for $S^{\prime}$ in Example 4.4(1). By specializing $w_{p p}$ (respectively, $w_{40}$ ) to $h$, one obtains the equations for $S_{h}$. A regular system of parameters for $B_{\eta}$ consists of $u_{i j}$ with $(i, j) \in D_{1} \cup D_{2}$ and $w_{p p}$ (respectively, $\left.\omega_{40}\right)$. By specializing, now in $\operatorname{Spec}\left(B_{\eta}\right)$, one gets one-codimensional smooth subschemes $\widetilde{S}_{h}$ of $\widetilde{S}_{R}^{\text {wes }}$ given by

$$
\left.w_{p p}-h=0 \quad \text { (respectively, } q_{40}-h=0\right) \text {. }
$$

Each $\widetilde{S}_{h}$ applies to $S_{h}$ in $\operatorname{Spec}(B)$. The deformation induced by $\eta$ on $\widetilde{S}_{h}$ is again semiuniversal for $\underline{\mathcal{D} e f}{ }_{R}^{\text {es }}$. The image of $T_{\bar{R} / R}^{1, \text { es }}$ in the tangent space to $\operatorname{Spec}\left(B_{\eta}\right)$ is the one-dimensional vector subspace $T$ generated by $\partial / \partial \omega_{p p}$ (respectively, $\left.\partial / \partial \omega_{40}\right)$. Each $\widetilde{S}_{h}$ is transversal to $T$. In fact, any hyperplane transversal of $T$ is realized as tangent space to some $\widetilde{S}_{h}$ for some convenient choice of $h$ of type $h=\sum_{(i, j) \in D} a_{i j} u_{i j}, a_{i j} \in K$.

Case 2: The embedded resolution consists of the blow-up of one point of multiplicity 4, three points of multiplicity 2 and two points of multiplicity 1 , with $e f_{R}=4$. One has $\operatorname{dim} S_{R}^{\text {wes }}=\operatorname{dim} T_{R}^{1, \mathrm{sec}}-17=7$, $\operatorname{dim} B_{R}^{\mathrm{es}}=\operatorname{dim} B_{\bar{R} \leftarrow R}^{\mathrm{es}}=5$. In particular, every $S_{h, h^{\prime}}$ is isomorphic to $\left(B_{R}^{\mathrm{es}}\right)$. The computation of $B_{\eta}$ gives rise to

$$
\begin{aligned}
u_{i j} & =0,(i, j) \notin\{(4,0),(4,2),(5,1),(6,0),(3,3),(4,3),(5,2),(5,3)\} \\
u_{40} & =w_{4,0}^{4} \\
u_{51} & =w_{4,0}^{2} u_{3,3} \\
u_{60} & =w_{6,0}^{2}+u_{5,1} w_{4,0}+u_{4,2}^{2}+u_{3,3} w_{4,0}^{3} \\
\left(1+w_{6,0}^{2}\right) u_{4,2} & =w_{4,2}^{2}+w_{6,0}+\left(1+w_{6,0}^{2}\right) u_{3,3} u_{4,0}^{3}+\left(1+w_{6,0}\right)\left(u_{4,3} w_{4,0}^{3}+u_{5,2} w_{4,0}^{2}\right) .
\end{aligned}
$$

By eliminating $w_{4,0}, w_{6,0}$, and $w_{4,2}$ one gets as equations for $S_{R}^{\text {wes,sec }}$ exactly those of $S$ in Example 4.4(2). By specializing $w_{4,0}, w_{6,0}, w_{4,2}$ to $h, h^{\prime \prime}, h^{\prime}$, respectively, one obtains the equations defining $S_{h, h^{\prime}}$. A regular system of parameters for $B_{\eta}$ is given by $u_{3,3}, u_{4,2}, u_{4,3}, u_{5,2}, u_{5,3}, w_{4,0}, w_{4,2}$. By specializing $w_{4,0}$ and $w_{4,2}$ one obtains smooth subschemes $\widetilde{S}_{h, h^{\prime}}$ of $S_{R}^{\text {wes,sec }}$ given by

$$
w_{4,0}-h=0, \quad w_{4,2}-h^{\prime}=0,
$$

which apply to $S_{h, h^{\prime}}$, are isomorphic to $\operatorname{Spec}\left(B_{R}^{\mathrm{es}}\right)$, and, moreover, the deformation induced by $\eta$ on them is semiuniversal for $\frac{\mathcal{D} e f}{R}$. All of the schemes $S_{h, h^{\prime}}$, are transversal to the image of $T_{\bar{R} / R}^{1, \text { es }}$ in $T_{B_{\eta}}$ which is nothing but the vector subspace generated by $\partial / \partial w_{4,0}$ and $\partial / \partial w_{4,2}$. The weak equisingularity stratum $S_{R}^{\mathrm{wes}, \mathrm{sec}}$ is singular in this case. 


\section{EQUISINGULAR DEFORMATIONS IN ARBITRARY CHARACTERISTIC}

Case 3: The embedded resolution consists of the blow-up of one point of multiplicity $p, l-1$ points of multiplicity 2 , and one point of multiplicity 1 , with $e f_{R}=2$. So, one has $\operatorname{dim} S_{R}^{\text {wes,sec }}=$ $\operatorname{dim}_{K} T_{R}^{1, \text { sec }}-\left(2 l^{2}+2 l-3\right)=(l-2)^{2}, \operatorname{dim}\left(B_{R}^{\text {es }}\right)=\operatorname{dim}\left(B_{\bar{R} \leftarrow R}^{\mathrm{es}}\right)=(l-2)^{2}$. The computation of $B_{\eta}$ gives rise to

$$
u_{i j}=0, \quad(i, j) \in D, i+j \leqslant p \text { or } i+j=p+1 \text { and } j \leqslant l-1,
$$

so one has $\operatorname{Spec}\left(B_{\eta}\right)=S_{R}^{\text {wes,sec }}=S^{\prime}$. On the other hand, the deformation induced by $\eta$ on $S^{\prime}$ is semiuniversal for $\frac{\mathcal{D} e f}{R}{ }_{R}^{\text {es }}$, so $S^{\prime}=S_{R}^{\text {wes,sec }}$ is the strong equisingularity stratum for $R$, which exists in this case.

Case 4: The embedded resolution consists of the blow-up of one point of multiplicity $p+1$, one point of multiplicity 2 , and $p-1$ points of multiplicity 1 , with $e f_{R}=2$. Then one has $\operatorname{dim} S_{R}^{\mathrm{wes}, \mathrm{sec}}=$ $\operatorname{dim}_{K} T_{R}^{1, \text { sec }}-\frac{1}{2}\left(p^{2}+5 p+2\right)=\frac{1}{2}(p-1)(p-2), \operatorname{dim}\left(B_{R}^{\text {es }}\right)=\operatorname{dim}\left(B_{\bar{R} \leftarrow R}^{\text {es }}\right)=\frac{1}{2}(p-1)(p-2)$. The computation of $B_{\eta}$ gives rise to

$$
u_{i, j}=0, \quad(i, j) \in D, i+j \leqslant p+1,
$$

so $\operatorname{Spec}\left(B_{\eta}\right)=S_{R}^{\text {wes,sec }}=S^{\prime}$, the deformation induced by $\eta$ on $S^{\prime}$ is semiuniversal for $\underline{\text { Def }}{ }_{R}^{\text {es }}$, and again $S^{\prime}=S_{R}^{\mathrm{wes}, \mathrm{sec}}$ is the strong equisingularity stratum, which exists in this case.

\section{ACKnowledgements}

Our collaboration on this paper was supported by the universities of Kaiserslautern and Valladolid and by a Research in Pairs stay at the Mathematisches Forschungsinstitut Oberwolfach. We would like to thank these institutions for their hospitality and support.

\section{REFERENCES}

Buc81 R.-O. Buchweitz, Contribution à la théorie des singularités, Thèse, Université Paris VII (1981).

Cam80 A. Campillo, Algebroid curves in positive characteristic, Lecture Notes in Mathematics, vol. 813 (Springer, Berlin, 1980).

GL07 A. Campillo, G.-M. Greuel and C. Lossen, Equisingular calculations for plane curve singularities, in Effective methods in algebraic geometry (MEGA 2005), ed. A. Dickenstein et al., J. Symbolic Comput. 42 (2007), 89-114.

Cas00 E. Casas-Alvero, Singularities of plane curves, London Mathematical Society Lecture Notes Series, vol. 276 (Cambridge University Press, Cambridge, 2000).

Del73 P. Deligne, Intersection sur les surfaces reguliers, in Séminaire de Géométrie Algébrique (SGA VII), Lecture Notes in Mathematics, vol. 340 (Springer, Berlin, 1973), 1-38.

Fle8l H. Flenner, Ein Kriterium für die Offenheit der Versalität, Math. Z. 178 (1981), 449-473.

GK89 G.-M. Greuel and U. Karras, Families of varieties with prescribed singularities, Compositio Math. 69 (1989), 83-110.

GLS07 G.-M. Greuel, C. Lossen and E. Shustin, Introduction to singularities and deformations, Springer Monographs in Mathematics (Springer, Berlin, 2007).

GP02 G.-M. Greuel and G. Pfister, A Singular introduction to commutative algebra (Springer, Berlin, 2002).

Hir63 H. Hironaka, On the equivalence of singularities, I, in Arithmetical algebraic geometry, West Lafayette, IN, 1963 (Harper and Row, New York, 1963), 153-200.

Laz73 F. Lazzeri, A theorem on the monodromy of isolated singularities, in Singularités à Cargèse (Rencontre sur les Singularités en Géométrie Analytique), Institut d'Études Scientifiques de Cargèse, 1972, Astérisque 7/8 (1973), 269-275.

Lip88 J. Lipman, On complete ideals in regular local rings, in Algebraic geometry and commutative algebra, Vol. I (in honour of M. Nagata) (Kinokuniya, Tokyo, 1988), 203-231. 
Mat86 H. Matsumura, Commutative ring theory (Cambridge University Press, Cambridge, 1986).

Sch68 M. Schlessinger, Functors of Artin rings, Trans. Amer. Math. Soc. 130 (1968), 208-222.

Tei78 B. Teissier, The hunting of invariants in the geometry of discriminants, in Real and complex singularities, Oslo, 1976, ed. P. Holm (North-Holland, Amsterdam, 1978).

Wah74a J. Wahl, Equisingular deformations of plane algebroid curves, Trans. Amer. Math. Soc. 193 (1974), 143-170.

Wah74b J. Wahl, Deformations of plane curves with nodes and cusps, Amer. J. Math. 96 (1974), 529-577.

Zar38 O. Zariski, Polynomial ideals defined by infinitely near base points, Amer. J. Math. 60 (1938), $151-204$.

Antonio Campillo campillo@agt.uva.es

Departamento de Algebra, Geometria y Topologia, Universidad de Valladolid, Facultad de Ciencias E-47005 Valladolid, Spain

Gert-Martin Greuel greuel@mathematik.uni-kl.de

Fachbereich Mathematik, TU Kaiserslautern, Erwin-Schrödinger-Straße, D-67663 Kaiserslautern, Germany

Christoph Lossen lossen@mathematik.uni-kl.de

Fachbereich Mathematik, TU Kaiserslautern, Erwin-Schrödinger-Straße, D-67663 Kaiserslautern, Germany 